\title{
Structure Formation with Ultralight Axion Dark Matter
}

\author{
Dissertation \\ for the award of the degree \\ "Doctor of Philosophy" \\ at the Georg-August-Universität Göttingen \\ within the doctoral programme PROPHYS \\ of the Georg-August University School of Science (GAUSS)
}

submitted by

Xiaolong Du

from Sichuan, China

Göttingen, 2018 


\section{Thesis advisory committee}

Prof. Dr. Jens C. Niemeyer

Institute for Astrophysics, Georg-August-University, Goettingen

Prof. Dr. Laura Covi

Institute for Theoretical Physics, Georg-August-University, Goettingen

\section{Members of the examination board:}

Referee:

Prof. Dr. Jens C. Niemeyer

Institute for Astrophysics, Georg-August-University, Goettingen

\section{Co-referee:}

Dr. David J. E. Marsh

Institute for Astrophysics, Georg-August-University, Goettingen

\section{Other members of the Examination Board:}

Prof. Dr. Wolfram Kollatschny

Institute for Astrophysics, Georg-August-University, Goettingen

Prof. Dr. Steffen Schumann

Institute for Theoretical Physics, Georg-August-University, Goettingen

Prof. Dr. Karl-Henning Rehren

Institute for Theoretical Physics, Georg-August-University, Goettingen

Prof. Dr. Eberhard Bodenschatz

Max Planck Institute for Dynamics and Self-Organization

Day of the oral examination: 24. 09. 2018 


\section{Acknowledgements}

First of all I would like to thank Prof. Dr. Jens Niemeyer for giving me the opportunity to work on this challenging and exciting project, for his continuous support, for providing an excellent working atmosphere, and for the scientific freedom he has granted me during the past years. His constructive suggestions, the great academic freedom, and his desire to gain a detailed understanding of physics have been wonderful motivations. I would also like to thank Prof. Dr. Laura Covi for co-supervising this dissertation and for helpful discussions.

The other members of my thesis committee and examination board are gratefully acknowledged for spending their precious time with my dissertation.

I would like to thank Christoph Behrens for his great help in the past four years, for carefully proof-reading my dissertation.

I would like to thank everybody in our group, especially David J. E. Marsh, Katy Clough, Erik Lentz, Bodo Schwabe, Jan Veltmaat, Philipp Grete, Dimitar Vlaykov, Benedikt Eggemeier, Jiajun Chen, Gaoyuan Wang, Jens Oltmanns, Felix Wiebe and Viraf M. Mehta for the great working atmosphere and a wonderful time in the office, and for being colleagues and friends.

The China Scholarship Council is acknowledged for financial support.

Finally, I want to thank my girlfriend, Ming Li and my family for their love and support. 


\begin{abstract}
Ultralight axion is a scalar field with an extremely small mass $\sim 10^{-22} \mathrm{eV}$. It is proposed as an alternative dark matter candidate to the standard cold dark matter (CDM). If self-interactions between axions can be ignored, it is also called fuzzy dark matter (FDM). On large scales, FDM behaves like CDM producing large scale structure of the Universe consistent with current observations. But on scales below the Jeans length, quantum pressure arising from coherent oscillations of the scalar filed counters gravity, leading to a large suppression in the structure formation and cored dark matter halo profiles. In this dissertation, I mainly discuss the cosmic structure formation in the scenario of FDM. First, I show how we implement FDM into the publicly available semi-analytic code for galaxy formation, GALACTICUS. With the modified code, we compute the (sub)halo mass function for FDM with different particle masses and density fractions. Comparing to the standard CDM, the (sub)halo mass function is found to be largely suppressed at lower masses. The suppression scale is inversely proportional to the square root of FDM mass. Then, based on the core mass growth in each binary merger of FDM halos, a simple model for the core-halo mass relation observed in simulations is proposed. The model is verified using the modified GALACTICUS code. Finally, to study tidal disruption of FDM subhalo cores, I perform idealized simulations using a fourth-order pseudo-spectral method. The core mass loss rate due to tidal stripping obtained numerically is compared with the previous results from semi-analytic treatments. We find after some reinterpretations, two results are reasonably consistent. Implementing the numerical results into GALACTICUS, I compute the subhalo mass function with and without considering the core stripping. Fitting formula for the subhalo mass function is given at the end, which will be useful in future studies on constraining FDM from observations related to halo substructure.
\end{abstract}




\section{Contents}

1 Introduction 1

$1.1 \quad$ Evidence of Dark Matter . . . . . . . . . . . . . . . . . . . . . . . . 1

1.1.1 Dynamics of Galaxy and Galaxy Cluster . . . . . . . . . . . . 1

1.1 .2 Expansion History of the Universe . . . . . . . . . . . . . . . 2

1.1 .3 Cosmic Microwave Background . . . . . . . . . . . . . . . 3

1.2 Dark Matter Models . . . . . . . . . . . . . . . . . . . . . . . . . 4

$1.2 .1 \quad$ QCD axion . . . . . . . . . . . . . . . . . 5

1.2 .2 Ultralight Axion Dark Matter . . . . . . . . . . . . . . . . 6

2 Semi-analytic Model of Structure Formation with FDM 9

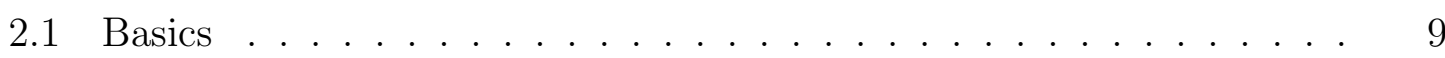

2.1 .1 Homogeneous and Isotropic Universe . . . . . . . . . . . . 9

2.1 .2 Linear Perturbations . . . . . . . . . . . . . . . . . . . . . 14

2.1 .3 Nonlinear Evolution in the Nonrelativistic Limit . . . . . . . . 17

2.1 .4 Spherical Collapse Model . . . . . . . . . . . . . . . . . . . . 18

2.1 .5 Press-Schechter Formalism . . . . . . . . . . . . . . . . . . . . 23

2.1 .6 Extended Press-Schechter Formalism . . . . . . . . . . . . . . 24

2.1 .7 Merger Tree . . . . . . . . . . . . . . . . . . . . . . . . . . . . . . 28

2.2 Semi-analytic Model of Galaxy Formation . . . . . . . . . . . . . . . 31

2.3 Implementation of FDM in SAMs . . . . . . . . . . . . . . . . . . 32

2.3 .1 A New Determination of the HMF for FDM . . . . . . . . . . 33

$2.3 .2 \quad$ Modification of the Tree Building Algorithm . . . . . . . . . . 40

2.3 .3 FDM Halo Profile . . . . . . . . . . . . . . . . . . . . . . . . . 41

2.3 .4 Satellite Model . . . . . . . . . . . . . . . . . . . . . . . . . . 43

2.3 .5 Modifications for FDM . . . . . . . . . . . . . . . . . . . . 45

2.4 Results for (Sub)halo Mass Function from SAMs . . . . . . . . . . . . 46

2.4 .1 Halo Mass Function. . . . . . . . . . . . . . . . . . . . . . 47

$2.4 .2 \quad$ Validating Merger Trees . . . . . . . . . . . . . . . . . . 51 
2.4 .3 Subhalo Mass Function . . . . . . . . . . . . . . . . . . . 52

2.5 Core Mass-Halo Mass Relation. . . . . . . . . . . . . . . . . . . . . 59

2.5 .1 Simplified Model . . . . . . . . . . . . . . . . . . 60

2.5 .2 Testing with Monte-Carlo Merger Trees . . . . . . . . . . . . 63

2.6 Conclusions and Discussions $\ldots \ldots \ldots \ldots \ldots \ldots$

\begin{tabular}{lll}
\hline 3 & Tidal Disruption of FDM Cores & 70
\end{tabular}

3.1 Classical and Tunneling Tidal Radius . . . . . . . . . . . . . . 71

3.2 Numerical Method . . . . . . . . . . . . . . . . . . . . . 75

$3.2 .1 \quad$ Pseudospectral Method . . . . . . . . . . . . . . . . . . . 75

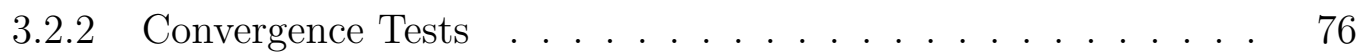

3.3 Core Mass Loss Rate from Simulation . . . . . . . . . . . . . . . . 77

3.3.1 Spherically Symmetric Approximation . . . . . . . . . . . 77

3.3 .2 Three-dimensional Simulation . . . . . . . . . . . . . . . . . 80

3.4 Tidal Locking $\ldots \ldots \ldots \ldots$

3.5 Satellite Galaxies in the Milky Way . . . . . . . . . . . . . . . 84

3.6 Effects on Subhalo Mass Function . . . . . . . . . . . . . . . . 86

3.7 Conclusions and Discussions $\ldots \ldots \ldots \ldots$

\begin{tabular}{lll}
\hline 4 & Outlook & 92
\end{tabular}

\begin{tabular}{|ll}
\hline A Numerical method for solving the integral equation & 93
\end{tabular}

\begin{tabular}{ll}
\hline Bibliography & 95
\end{tabular} 


\section{List of Figures}

2.1 Evolution of the scalar field in a homogeneous universe. Left: potential of the scalar field. The solid curve shows the approximated axion potential up to the quadratic term. The vertical dashed line marks the time when axion begins oscillating and behaves as dark matter. Right: state parameter of the scalar field. . . . . . . . . . . . . . . . . 12

2.2 Spherical collapse. Left: evolution of the radius of the overdense sphere (solid line) compared to the background expansion (dashed line). In the realistic case, the sphere will not collapse to a singularity but virialize via phase mixing and violent relaxation (indicated by the shaded region). Right: evolution of the overdensity $\delta$ (solid line) compared to the prediction from the linear perturbation theory $\delta_{\text {lin }}$. At the beginning, $\delta$ is small and grows just like $\delta_{\operatorname{lin}}$. As $\delta$ increases, it enters the nonlinear regime and grows faster than $\delta_{\text {lin }}$. At the time $t_{m}$ when the radius of the overdense sphere reaches its maximum, $\delta \approx 4.28 \delta_{\operatorname{lin}} . \quad$. $\quad 19$

2.3 Simulations of spherical collapse with FDM. Left: density profile at the collapse time for different initial overdensities. $\rho_{m 0}$ is the background matter density at current time. The initial overdensity is taken to be a single spherical Fourier mode, i.e. $\delta\left(x, t_{i}\right)=\delta_{i} \sin \left(k_{s} x\right) /\left(k_{s} x\right)$. Two simulations have the same $\delta_{i}=0.02$ but different $k_{s}$. Right: evolution of the central density $\rho_{c}$. The collapse time is marked by the vertical lines. . . . . . . . . . . . . . . . . . . . 22

2.4 A realization of the trajectory of $\delta_{R} \ldots \ldots \ldots 26$

2.5 A schematic merger tree. . . . . . . . . . . . . . . . . 28

$2.6 \quad$ A schematic diagram of semi-analytic model of galaxy formation. . . 32 
2.7 Transfer function $T_{\mathrm{FDM}}(k)$, as defined in Eq. (2.88), for FDM with different masses and fractions. The results is derived using the publicly available code AxionCAMB. Here we have also considered a model in which FDM only makes up half of the dark matter, i.e. $\Omega_{a} / \Omega_{d}=0.5$ (thick dashed curve). The vertical dashed lines mark the Jeans wave numbers at matter-radiation equality for different FDM masses. . . . 35

2.8 Critical overdensity for collapse at $z=0$ with different FDM fractions \begin{tabular}{|l|l|}
\hline$\Omega_{a} / \Omega_{d}$. The fractions range from 0 to 1 with a step size of 0.1 . The \\
\hline \hline FDM mass is set to $10^{-22} \mathrm{eV}$. Reproduced from [1], Fig. 1. . . . . . . 38
\end{tabular}

2.9 Critical overdensity for collapse at $z=0$ with different FDM masses

\begin{tabular}{|c|}
\hline (based on the fitting formula from [2]) compared to standard CDM. \\
\hline
\end{tabular}
Reproduced from [1], Fig. 2. . . . . . . . . . . . . . . . . 38

$2.10 \mathrm{HMF}$ for FDM fraction $f=1.0$ (solid lines) compared to standard \begin{tabular}{|l|}
\hline CDM (dashed lines). Redshifts range from $z=14$ to 0 , obtained using \\
\hline \hline our new calculation detailed above (left panel) and the Sheth-Tormen \\
\hline formalism (right panel). The FDM mass is set to $m_{\mathrm{a}}=10^{-22} \mathrm{eV}$. \\
\hline Reproduced from [1] Fig. 3.
\end{tabular} Reproduced from [1], Fig. 3. . . . . . . . . . . . . . . . . . 47

$2.11 \mathrm{HMF}$ at $z=0$ with different FDM fractions $f$. The fractions range \begin{tabular}{|c|c|}
\hline from 0 to 1 with a step size of 0.1 . The FDM mass is set to $m_{\mathrm{a}}=$ \\
\hline
\end{tabular} $10^{-22} \mathrm{eV}$. Reproduced from [1], Fig. 4. . . . . . . . . . . . . . . 48

2.12 HMF at $z=0$ with different FDM masses compared to standard CDM. Reproduced from [1], Fig. 5. . . . . . . . . . . . . . . . . 49

2.13 Halo mass function for FDM computed from different approaches com-

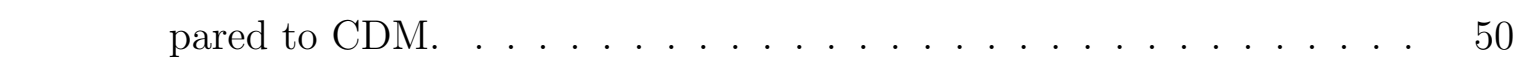

2.14 Cumulative number density of halos with $M>10^{6} M_{\odot}$ at $z=6$ cal\begin{tabular}{|c|c|}
\hline culated from different HMFs compared to observation [3]. $m_{22} \equiv$ \\
\hline
\end{tabular} $m_{a} /\left(10^{-22} e V\right)$. . . . . . . . . . . . . . . 51

2.15 HMF from merger trees at $z=0$ for different models. Symbols indicate \begin{tabular}{l}
\hline the data from the merger trees, the lines indicate the directly calcu- \\
\hline lated HMF. Left: HMF from merger trees for pure FDM and MDM \\
\hline compared to standard CDM. The FDM mass is set to $m_{a}=10^{-22} \mathrm{eV}$. \\
\hline Right: HMF from merger trees for pure FDM with different masses \\
\hline compared to standard CDM. Reproduced from [1], Figs. 6 and 7. . . \\
\hline
\end{tabular} 
$2.16 \mathrm{HMF}$ from merger trees at $z=3$ for different models. Left: HMF from merger trees for pure FDM and MDM compared to standard CDM. The FDM mass is set to $m_{\mathrm{a}}=10^{-22} \mathrm{eV}$. Right: HMF from merger trees for pure FDM with different masses compared to standard CDM. Reproduced from [1], Figs. 8 and 9. . . . . . . . . . . . . . . . 53

2.17 Cumulative distribution of the redshifts at which $z=0$ subhalos became subhalos for pure FDM $(f=1)$ with $m_{\mathrm{a}}=10^{-22} \mathrm{eV}$. Reproduced from [1], Fig. 10. . . . . . . . . . . . . . . . . . . . . . . . 54

2.18 SHMF from merger trees for standard CDM. 'Fric' refers to dynamic friction and 'Strip' refers to tidal stripping. Reproduced from [1], Fig.

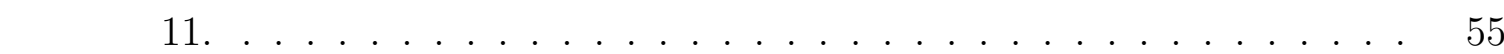

2.19 SHMF from merger trees for MDM with $\Omega_{a} / \Omega_{d}=0.5$ (left) and pure FDM. The FDM mass is set to $m_{\mathrm{a}}=10^{-22} \mathrm{eV}$. Modified from [1], Figs. 12 and $13 . \ldots \ldots \ldots \ldots \ldots 6 . \ldots \ldots \ldots$

2.20 SHMF for FDM and MDM $(f=0.5)$ using the "orbiting" implementation with dynamical friction and tidal stripping compared with the standard CDM. The FDM mass is set to $m_{\mathrm{a}}=10^{-22} \mathrm{eV}$. Modified from [1], Figs. 14 and 15. . . . . . . . . . . . . . . . . 57

2.21 SHMF for FDM with and without modification to concentration parameter. The FDM mass is set to $m_{\mathrm{a}}=10^{-22} \mathrm{eV}$. Reproduced from [1], Fig. 16. . . . . . . . . . . . . . . . . . . . . . 58

2.22 An example of a merger tree with 7 major mergers (solid lines) and 2 minor mergers (dashed lines). The dotted lines represent the smooth accretion. Note that the size of the circle represents the mass of the core (not necessarily in a consistent scale). . . . . . . . . . . . . . . 62

2.23 Evolution of core mass and halo mass for several merger trees. Only \begin{tabular}{|c|}
\hline the main branch is shown. The straight line up-left shows the Schive \\
\hline
\end{tabular} et al. core-halo mass relation with arbitrary normalization as a reference. 64

2.24 The core mass with respect to the halo mass at $z=0$ for different initial core-halo mass relation: $n=1 / 3$ (left), $n=1$ (center), and $n=2$ (right). The dashed line shows the core-halo mass relation from [4], $n=1 / 3$, at $z=0$. The solid lines show the linear and square relations for comparison. Reproduced from [5], Fig. 1. . . . . . . . . 65 
2.25 Left: the halo mass with respect to the number of first-formed halos.

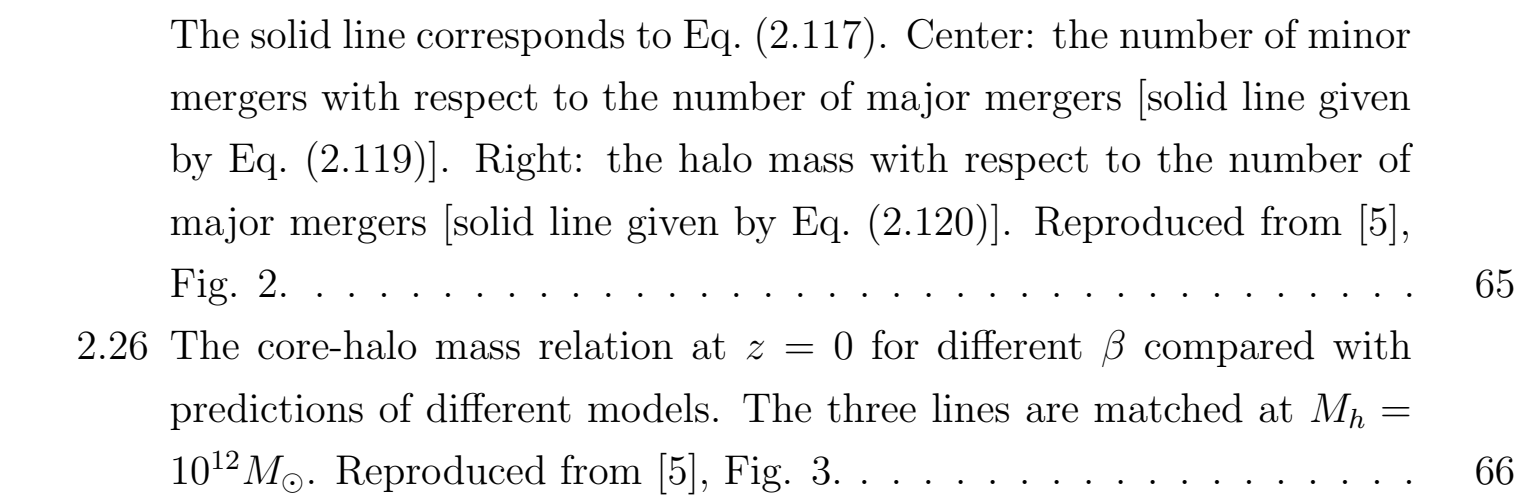

3.1 The imaginary part of the energy eigenvalue $E$. The solid line show the fitting formula Eq. (3.7). The crosses show the results from solving the eigenvalue problem as in [6]. . . . . . . . . . . . . . . . 73

3.2 Evolution of the core's central density with unevolved (dots) and evolved (crosses) gravitational potential compared to the prediction in [6]. Reproduced from [7], Fig. 1. . . . . . . . . . . . . . . . . . . . . . . . 74

3.3 Regions inside (blank region) and outside (shaded region) the tidal radius with respect to the density ratio $\mu \equiv \rho_{c} / \bar{\rho}_{\text {host }}$. The solid line shows the tidal radius computed from Eq. (3.3). The horizontal lines mark the radii enclosing $95 \%$ (top), $50 \%$ (middle) and 25\% (bottom) of the total soliton mass, respectively. When $\mu<4.5$, the tidal radius is smaller than the core radius. Thus, the solitonic core becomes unstable and is quickly disrupted. Reproduced from [7], Fig. 2. . . . . . . . . 74

3.4 Numerical error of the total energy with respect to time for different time step sizes and different algorithms. The time is in units of the free-fall time scale $t_{\mathrm{ff}}=\sqrt{3 \pi /(32 G \rho)}$ with $\rho$ equal to the average density over the whole simulated box. Here "O4" refers to the fourthorder algorithm we used in our simulations (Eq. 3.13$)$. "O2" refers to the second-order kick-drift-kick formulation Eq. (3.12), which is widely used in previous simulations. Reproduced from [7,, Fig. 11. . . . . . . 77

3.5 Average numerical error of the total energy with respect to the time step size. Only data with $t>3 t_{\mathrm{ff}}$ when the numerical error oscillates around roughly a constant value is included in the analysis. Reproduced from [7], Fig. 12. . . . . . . . . . . . . . . . . . 78 
3.6 Mass loss rate for different initial density ratios. A spherical tidal field is assumed. The solid black line shows the prediction in [6] using the fitting formula Eq. (3.7). The vertical dashed lines are the same as in Fig. 3.3. The solid lines with different colors give the total mass loss rate. The dashed colored lines show the mass loss rate by mass transfer through the tidal radius, while the shaded region corresponds to the mass loss rate from the decreasing tidal radius. Reproduced from [7], Fig. 3. . . . . . . . . . . . . . . . . . . . . . 79

3.7 The density profile of the core at the initial time and at $t=4 T_{\text {orbit }}$ \begin{tabular}{|l|}
\hline for a density ratio $\mu=50$. The circles and squares show the average \\
\hline radial density profile obtained from the simulation. The lines display \\
\hline fitted profiles defined in Eq. (3.8). It can be seen that the cores are \\
\hline well described by soliton profiles even after losing substantial amounts \\
\hline of mass. Reproduced from [7] Fig. 4. . . . . . . . . . . . 81
\end{tabular}

3.8 Slices through the density field at different times for an initial density ratio $\mu=50$. The circle in the center of each plot indicates the size of the host (for simplicity the host is treated as a small sphere with uniform density). The thick and thin contour lines mark where the density drops to $50 \%$ (core radius) and $1 \%$ of the maximum density, respectively. The dashed circles show the tidal radii computed from the spherically symmetric approximation. Reproduced from [7], Fig. 5.$] 82$

3.9 Core mass loss rate for different initial density ratios. The lines show the prediction from Eq. (3.7) and Eq. (3.17) with $\gamma=3 / 2$ (solid line) and $\gamma=1$ (dashed line). The vertical dashed lines are the same as in Fig. 3.3. Reproduced from [7], Fig. 6. . . . . . . . . . . . . . . . . . . 82

3.10 Slice through the core. The color map indicates the density while

\begin{tabular}{l}
\hline the black arrows trace the velocity field relative to the core's collective \\
\hline motion. Inside the tidal radius (dashed circle) the velocity field is char- \\
\hline acteristic for an irrotational Riemann-S ellipsoid [8]. Outside the core, \\
\hline vortices can be seen. The thick black circle in the middle represents \\
\hline the core radius while the thin black ellipsoid marks the area where the \\
\hline density drops to one percent of the central density, i.e. almost all the \\
\hline mass lies within the tidal radius. The hollow arrow points towards the \\
\hline host's center. Reproduced from [7], Fig. 7. . . . . . . . . . . . . . 83
\end{tabular}


3.11 Representative run with initial density ratio $\mu=50$. Top: angle between the longest principal axis of the core and $x$-axis (grey circles).

\begin{tabular}{l}
\hline The dashed line shows the angle between the line joining the center of \\
\hline the core and the center of host and $x$-axis. It can be seen that the core \\
\hline is tidally locked most of the time. Center: eccentricity $\epsilon_{13}$ of the core. \\
\hline The solid line shows the expected values from Eq. (3.18). Bottom: spin \\
\hline parameter of the core. Reproduced from [7], Fig. 8. . . . . . . . . . . 85 \\
\hline
\end{tabular}

3.12 Minimum mass of cores that can survive for $N_{\text {sur }}$ orbits assuming different FDM particle masses $m_{22} \equiv m /\left(10^{-22} \mathrm{eV}\right)$ versus the distance \begin{tabular}{|l|}
\hline to the Galactic center $D$. For comparison, we also show the half-light \\
\hline \hline mass $M_{1 / 2}$ of some satellite galaxies in the Milky Way [9]. The mass \\
\hline of the host is taken to be $10^{12} M_{\odot}$. For each particle mass, the solid \\
\hline curve is obtained by assuming $\gamma=1$ and $N_{\text {sur }}=1$ while the dashed \\
\hline curve is obtained by assuming $\gamma=3 / 2$ and $N_{\text {sur }}=10$. To survive \\
\hline for $N_{\text {sur orbits, the half-light mass of satellite galaxies should be above }}$ \\
\hline the lines (assuming core mass is at most equal to the half-light mass). \\
\hline Reprodued from
\end{tabular} Reproduced from [7], Fig. 9. . . . . . . . . . . . . . . . . 86

3.13 SHMF for different FDM masses. The solid and dashed curves are from

\begin{tabular}{|c|}
\hline the fitting functions Eqs. (3.22) and (3.25). Left: with tidal stripping \\
\hline of
\end{tabular}

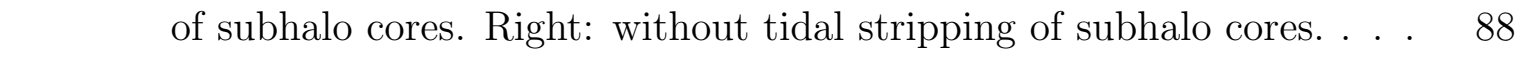

3.14 Robustness test of the fitting formula Eq. (3.22) for FDM with (solid \begin{tabular}{|l|}
\hline line) and without (dashed line) tidal stripping of subhalo cores. The \\
\hline \hline
\end{tabular} \begin{tabular}{l}
\hline FDM mass $m_{22}=m_{a} /\left(10^{-22} \mathrm{eV}\right)=50$. The solid and dashed lines \\
\hline show the prediction from the fitting formula. Note that the fitting \\
\hline formula is derived from the results of merger trees with $m_{22}=1-10$ \\
\hline and extrapolated to $m_{22}=50$. . . . . . . . . . . . . . . . . 89
\end{tabular}

A.1 HMF for FDM fraction $f=1.0$ derived using the mid-point rule (solid \begin{tabular}{|l|}
\hline lines) compared to the result using the trapezoid rule (dashed lines). \\
\hline Redshifts range from $z=14$ to 0 . The FDM mass is set to $m_{\mathrm{a}}=$ \\
\hline $10^{-22} \mathrm{eV}$. Reproduced from [1], Fig. A1. . . . . . . . . . . . . . . . 94
\end{tabular} 


\section{Chapter 1}

\section{Introduction}

One of the fundamental questions in cosmology is how the Universe began and evolved into a complex system containing a lot of structure on different scales, from galaxy clusters, galaxies to stars. A variety of observations indicate that our Universe contains a large amount of "invisible" matter, i.e. dark matter. Dark matter is "dark" in the sense that it nearly does not interact with light, thus can not be seen directly by eyes or through telescopes. However, we do know its existence by the gravity it acts on other visible objects. Since it makes up about 26 percent of the current Universe, which is five times as much as the ordinary baryonic matter 1 , it plays an important role in the formation of cosmic structure. However, we have only inferred its existence through gravitational effects so far and known little about its nature. Different kinds of models for dark matter have been proposed motivated either by observations or by some theoretical considerations, e.g. an extension of the Standard Model of particle physics. In this dissertation, I will mainly concentrate on one of the many promising candidates, ultralight axion dark matter or as is commonly called fuzzy dark matter (FDM), and discuss the cosmic structure formation in this model.

\subsection{Evidence of Dark Matter}

\subsubsection{Dynamics of Galaxy and Galaxy Cluster}

One way to determine the mass of a distant system is to observe the movement of objects within this system. Then from Newton's law of gravity, the mass that is needed to reproduced such a movement can be calculated. If the system is in equilibrium, we can further use the virial theorem to get the relation between the

\footnotetext{
${ }^{1}$ As is commonly used in cosmology, in this thesis the word "baryonic matter" or "baryons" refers to everything that is made up of protons, neutrons and electrons.
} 
velocity dispersion (or kinetic energy) and the total mass and size of the system (or the potential energy). Adopting the virial theorem, in 1933 Fritz Zwicky first computed the mass of the Coma Cluster from the observed velocity dispersion in the movements of galaxies within the cluster. He found that the mass he obtained is 400 times larger than the mass expected from luminosity measurements [10]. It provided the first evidence that there might be a large amount of invisible matter in the Coma Cluster.

On the other hand, for spiral systems, the mass distribution can be inferred from the rotation velocity of visible stars or gas around the galaxy center by applying Kepler's second law. In the 1970s, the improvement in observation techniques made it possible to measure the rotation velocity of galaxies accurately to large radii with both optical and radio telescopes. In 1978, Rubin, Ford and Thonnard published their famous paper [11], in which they showed the rotation curves for ten spiral galaxies and found they are flat even out to the outermost radii that are measured. These results are inconsistent with the predictions from Newtonian dynamics: the rotation velocity $v=\sqrt{G M(r) / r}$; if there are only luminous matter (stars and gas) in the galaxy, as the density of luminous matter drops off at large radii the enclosed mass $M(r)$ approach to a constant, thus $v$ will drop as $1 / \sqrt{r}$. To resolve the inconsistency, additional invisible matter, i.e. dark matter, needs to be added. To see a full history I refer the interested readers to [12].

\subsubsection{Expansion History of the Universe}

Another evidence of dark matter is from measuring the expansion history of the Universe. As is first discovered by Hubble in 1929 [13], the Universe is expanding. The gravity force is attractive, so the expansion of the Universe will be slowed down by matter components. The more the matters there are, the larger the deceleration is. Therefore, by measuring the expansion of the Universe, we can derive the average matter density in the Universe and check whether it is consistent with the ordinary baryonic matter density we observe.

One way to measure the expansion history of the Universe is to look at type Ia supernovae. Type Ia supernovae produce consistent peak luminosity during their explosions, thus can be used as standard candles 2. Comparing the brightness of a supernova with its intrinsic luminosity, its distance to us can be derived using Gauss's law, i.e. the brightness decreases inversely proportional to the square of distance. On

\footnotetext{
${ }^{2} \mathrm{~A}$ standard candle is an object whose luminosity is well known.
} 
the other hand, the redshift of the supernova can be obtained from its spectrum. The redshift tells us how much the Universe has expanded since the explosion of a supernova. Comparing this expansion factor with distances to different supernovae gives the information about the expansion history, which then tells us about the average matter density. In 1998, two groups, the High-Z Supernova Search Team [14] and the Supernova Cosmology Project [15], discovered independently that the expansion of the Universe is actually accelerating and the Universe is made up of about $1 / 4$ of matter and 3/4 of dark energy (vacuum energy). As a comparison, the fraction of baryonic matter estimated from cosmic nucleosynthesis is less than $5 \%$. It indicates that there must be a dark matter component.

Another way to measure the expansion history is to search for the signature of Baryon Acoustic Oscillations (BAOs), i.e. density peaks and valleys caused by sound waves propagating in the primordial plasma. Before recombination, baryons coupled with photons giving rise to an effective pressure. The sound wave generated during the collapse of overdensities traveled at a speed of $c / \sqrt{3}$ with $c$ the speed of light. After recombination, the universe became neutral. Baryons decoupled with photons and became nearly pressureless. So the sound wave was frozen leaving behind an overdense shell at a fixed radius, i.e. the sound horizon. Thus it is expected to see a peak in the correlation function of the density field at the scale of sound horizon. At later times (lower redshifts), overdense regions collapse and form galaxies. The peak should still be seen in the correlation function of the number density field of galaxies, but the position of the peak has been moved to a larger scale due to the expansion of the Universe. Comparing it with the sound horizon at recombination, information about the expansion history can be obtained. Again the measurement of BAOs supports the existence of dark matter [16].

\subsubsection{Cosmic Microwave Background}

In the early Universe, it was so hot that electrons can not be bound to nuclei. Photons frequently interacted with free electrons making them in thermal equilibrium. The photons presented a black-body spectrum. As the universe expanded, the temperature decreased. Finally, at recombination free electrons are bound to nuclei again, making the Universe neutral and transparent to photons. Thus photons began to expand freely. They kept the black-body spectrum but with a decreasing temperature. At the present time, they become the cosmic microwave background (CMB) that fills in the Universe. 
The temperature of CMB is found to be nearly isotropic with only a very small variation across the sky. But it is these small anisotropies that contain a lot of important information about the Universe. They are not only relevant to studying the expansion of the Universe, but also relevant to studying the evolution of small fluctuations in different matter components. Unlike baryonic matter, dark matter has no interactions with photons except for the gravitational force. Its density perturbations evolved very differently from baryonic matter in the early Universe. Therefore, it is possible to distinguish dark matter from ordinary baryonic matter by measuring the angular power spectrum of CMB anisotropy. Since the first discovery of CMB by Arno Penzias and Robert Woodrow Wilson in 1964 [17], great improvements have been made [18, 19]. With the Planck satellite, 5 of the 6 parameters in the standard cosmological model can be measured better than 1 percent [20]. The latest results from Planck satellite indicate that dark matter makes up about 26 percent of the current Universe while the baryonic matter only makes up 5 percent.

\subsection{Dark Matter Models}

As is shown in the last section, dark matter is necessary to explain the discrepancy between the matter density estimated from gravitational effects and the density of normal baryonic matter. But so far we have not specified what particle it is.

From the observation point of view, it would be sufficient for most cases to assume that the dark matter is

(1) cold, which means its thermal velocity must be very small;

(2) dark, so it can at most only weakly interacts with photon, baryonic matter and itself.

This is the so-called cold dark matter (CDM) model. CDM together with a non-zero cosmological constant, i.e. the $\Lambda$ CDM model, has been successful in explaining a lot of current observations. From the particle physics point of view, however, such kind of a model may seem a bit primitive because it does not tell too much about the properties of the dark matter particle. We only know it is neutral and it is stable on cosmological time scales. But we do not even know its mass. As is discussed in [21], although there may be big differences when we see the dark matter problem from these two different aspects, they are actually closely related. On the one hand, any non-trivial property of dark matter particle could possibly predict a new phenomenon that can be tested by observations. On the other hand, if we observe some deviations 
from the CDM model, it would imply that the dark matter particle has some new properties that may be detected in particle physics experiments.

Since CDM has been well tested on scales larger than $10 \mathrm{kpc}$ and shows excellent agreements with observations of large scale structure of the Universe. The deviations from CDM are most likely to happen on small scales. It motivates various kinds of alternative models such as warm dark matter [22, 23] with non-negligible thermal motions and self-interacting dark matter [24] with measurable non-gravitational interactions. They can be realized as variants of the weakly interacting massive particles (WIMPs) dark matter paradigm. On the other hand, light (sub-eV), coherently oscillating scalar fields also behave like CDM on large scales while showing new phenomenology on small scales, starting roughly at their virial velocity de Broglie wavelength [25, 26, 27]. For particle masses around $10^{-22} \mathrm{eV}$, the length is of the order of several kpc, making this mass range sensitive to constraints from observations on galactic scales [28, 29, 30, 31, 32, 33, 34, 35, 1].

One of the most significant differences between these alternative dark matter models and the CDM model is the cosmic structure formation on small scales. N-body cosmological simulations show that CDM halos have cuspy cores [36] and contain a lot of small subhalos [37, 38]. On the contrary, the alternative models mentioned above may produce flat cores in halo centers. The abundance of subhalos may also be different on small scales. Thus searching for deviations from the predictions of the CDM model on small scales, e.g. substructure of dark matter halos, provides a promising way to constrain the properties of dark matter candidates.

\subsubsection{QCD axion}

Before going into more details about the main topic of this dissertation, the ultralight axion dark matter, it is worthwhile having a very brief introduction to the QCD axion, where the axion particle is first proposed.

In QCD Lagrangian, there exists a natural term that violates the combination of charge conjugation symmetry $(\mathrm{C})$ and parity symmetry $(\mathrm{P})$

$$
\mathcal{L}_{\theta}=\theta \frac{g^{2}}{32 \pi^{2}} F_{a}^{\mu \nu} \tilde{F}_{a \mu \nu}
$$

where $F_{a}^{\mu \nu}$ is the gluon field strength tensor and $\tilde{F}_{a \mu \nu}=\frac{1}{2} \epsilon_{\mu \nu \alpha \beta} F_{a}^{\alpha \beta}$ with $\epsilon_{\mu \nu \alpha \beta}$ the Levi-Civita symbol. If the parameter $\theta$ does not equal to 0 , this term will contribute to the neutron electric dipole moment $d_{n}$. The current measurement of $d_{n}$ suggests 
that $d_{n}<2.9 \times 10^{-26} \mathrm{ecm}$ [39], which implies $\theta$ is extremely small, $\theta \lesssim 10^{-10}$ [40]. So we will need a fine tuning for $\theta$. This is the so-called strong CP problem.

In 1977, Peccei and Quinn proposed a solution to the strong CP problem by introducing a global chiral $U(1)$ symmetry, which is also known as the Peccei-Quinn (PQ) symmetry [41]. The parameter $\theta$ is effectively replaced by a dynamical field $\varphi$ called axion, the Goldstone boson of spontaneously broken PQ symmetry:

$$
\mathcal{L}_{\theta, \varphi}=\left(\xi \frac{\varphi}{f_{a}}-\theta\right) \frac{g^{2}}{32 \pi^{2}} F_{a}^{\mu \nu} \tilde{F}_{a \mu \nu}
$$

where $\xi$ is the colour anomaly, and $f_{a}$ is the axion decay constant. Note that we have not shown the kinetic term for $\varphi$ here. Non-perturbative topological fluctuations of gluon fields induce an effective potential for $\varphi$ via the above terms. At the minimum of the effective potential, $\varphi=\frac{f_{a}}{\xi} \theta$, setting the $\mathrm{CP}$ violating term to 0 [42]. So the strong CP problem is naturally solved. The mass of QCD axion is given by ${ }^{3}[43,44]$

$$
m_{a} \approx 6 \times 10^{-6} \mathrm{eV}\left(\frac{10^{12} \mathrm{GeV}}{f_{a} / \xi}\right)
$$

Since QCD axion was first proposed, it has draw a lot of attentions, not only because it provides an elegant solution to the strong CP problem, but also because it can be a good candidate for dark matter [45, 46, 47, 48]. Typical QCD axion has a mass of $4 \times 10^{-10} \mathrm{eV} \sim 4 \times 10^{-2} \mathrm{eV}$.

\subsubsection{Ultralight Axion Dark Matter}

In the last subsection, I have briefly introduced the QCD axions whose masses are very small. As dark matter candidates, these QCD axions will mostly behave like collisionless CDM. In this subsection, I will continue with the ultralight axion(-like) particles whose masses are even smaller. Their masses can be $10 \sim 20$ orders of magnitude smaller than QCD axions.

It has been shown that ultralight scalar fields can be a viable candidate for dark matter if they are in a very cold state (e.g., [49, 50, 51, 26, 25, 52, 53, 54]). If consisting of particles of mass $\sim 10^{-22} \mathrm{eV}$ [55, 29, 4, 32, 31, 33, 40, 34, 56, 57] these candidates can potentially solve the well-known problems faced by the pure CDM model on small scales 4 (see [6] for a recent review):

\footnotetext{
${ }^{3}$ Note that the accurate form may depend on specific models.

${ }^{4}$ Nowadays, people also begin to think whether these may actually not be problems with the CDM model, but rather problems with limitations in observations and our knowledge of baryonic feedback.
} 
(1) Missing Satellites Problem [37, 38], number of satellite galaxies observed in the Milky Way is much less than the prediction from N-body simulations of CDM;

(2) Cusp-Core Problem [36], observations infers that low-mass dark matter halos have cored profiles instead of cuspy profiles as seen in CDM simulations;

(3) Too-Big-To-Fail Problem [58, the most massive dark matter subhalos in CDM simulations are too dense to host the brightest satellite galaxies observed in the Milky Way; in other words, theses most massive dark matter subhalos are so big that they should not fail to form bright galaxies that we can observe.

Possible constituents of such light scalar particles are ultralight axions (ULAs) that are produced nonthermally via the misalignment mechanism [59, 60, 61]. Unlike CDM which produces cuspy halo profiles, FDM produces flat halo cores [29, 62, 63. on scales smaller than the so-called quantum Jeans length [25, 27]. Below this scale, quantum effects suppress gravitational collapse.

By performing Jeans analysis, it is found in [29] that the cored halo profile corresponding to FDM with mass $m_{a}=0.81 \times 10^{-22} \mathrm{eV}$ can well reproduce the radial distribution of stars and their velocity dispersion in the Fornax dwarf spheroidal (dSph) galaxy. Further analysis on multiple stellar subpopulations in the Fornax and Sculptor dSph galaxies is done in [31] and an upper bond, $m_{a}<1.1 \times 10^{-22} \mathrm{eV}$, on the FDM mass is found by assuming that FDM alone can resolve the cusp-core problem. A similar constraint is found in [64] from Jeans analysis of eight classical dSph galaxies. In [57], it is demonstrated that Jeans analysis may be biased due to uncertainties in the assumed halo profile. Instead, a more stringent unbiased constraint, $m_{a}<0.4 \times 10^{-22} \mathrm{eV}$, is obtained in [57] by analyzing the averaged velocity dispersion of dSph galaxies.

Coherent oscillations of FDM also lead to a sharp suppression in the matter power spectrum [25] and halo formation [55, 33, 1, 65] below the Jeans scale. In turn, this cutoff scale for FDM halos puts a lower bound on the FDM mass since deviations from CDM cannot violate the constraints given by current observations. Using the cosmic microwave background and galaxy clustering data, 66 find a lower bound on the FDM mass, $m_{a} \gtrsim 10^{-24} \mathrm{eV}$. Constraints from UV luminosity functions and reionization are much tighter, e.g. [65] find $m_{a} \gtrsim 1.6 \times 10^{-22} \mathrm{eV}$ (see also [32] and [33]). This lower bound is in tension with the upper bound obtained from dwarf galaxies. Furthermore, the Ly $\alpha$ forest can also put a tight constraint on the FDM 
mass similar to the case of warm dark matter (WDM) [67, 55, 68]. For example, the latest observations of the Lyman-alpha forest have been interpreted as implying $m_{a}>2 \times 10^{-21} \mathrm{eV}[69,70]$, but systematic uncertainties are still disputed [71]. Thus, FDM may also suffer from the Catch 22 problem [72] like WDM, i.e. either producing too small halo cores or too few low-mass halos. However, as discussed in [57], to get more consistent constraints we need to consider details of the interplay between FDM and baryonic physics. The baryonic feedback may help reconcile the tension between different observations 73 .

This dissertation is organized as follows. Chapter 2 mainly shows our work on the semi-analytic model of structure formation for FDM. In Section 2.1, I first introduce some basic knowledge and tools that are widely used in cosmology. Then I discuss a bit about the semi-analytic model for galaxy formation in Section 2.2. In Section 2.3. I show how we implement the FDM model into the semi-analytic code for galaxy formation, GALACTICUS. Results for FDM (sub)halo mass functions are presented in Section 2.4. Finally, in Section 2.5. I show our new model for the core mass-halo mass relation, then conclude in Section 2.6. We note that Section 2.3, Section 2.4 and Section 2.5 are based on our published paper [1] and [5], but also include some of our new results.

Chapter 3 mainly shows our work on numerically simulating the tidal disruption of subhalo cores. First, in Section 3.1, I discuss the tidal radius in the classical and "quantum tunnelling" scenarios, respectively. Then I show the numerical algorithm we use to do the simulations and convergence tests in Section 3.2. Sections 3.33 .5 show our numerical results for the core mass loss rate. In Section 3.6, I show how the core stripping affects the subhalo mass function. Finally, conclusion and discussion are present in Section 3.7. Chapter 3 is based on our published paper [7]. Again, I have added some new results.

In Chapter 4. I provide an outlook on how my current work can be extended to some new projects in the future. 


\section{Chapter 2}

\section{Semi-analytic Model of Structure Formation with FDM}

\section{$2.1 \quad$ Basics}

\subsubsection{Homogeneous and Isotropic Universe}

The cosmological principle states that viewed on sufficiently large scales, the Universe is homogeneous and isotropic. It may look unreasonable at first glimpse. We do live in an inhomogeneous environment because we do see different stars, galaxies in different directions. However, with the help of the latest data from large galaxy surveys such as WiggleZ1 and SDSS-III/BOSS ${ }^{2}$, it has been shown that on scales larger than a few hundred Mpc the galaxies are distributed homogeneously [74, 75]. Another evidence is the measurement of Cosmic Microwave Background (CMB), which is the radiation left by the early Universe. The temperature of $\mathrm{CMB}$ is found to be around $2.7 \mathrm{~K}$. The variation across the whole sky is only about $10^{-5}$ [76], which means the matter distribution in the early Universe is isotropic on large scales. Thus as the simplest model, we first neglect all cosmic structure on small scales and treat the Universe as homogeneous and isotropic.

A homogeneous and isotropic universe is described by the well-known FriedmannLemaître-Robertson-Walker (FLRW) metric

$$
g_{\mu \nu} d x^{\mu} d x^{\nu}=-d t^{2}+a(t)^{2}\left(\frac{1}{1-k r^{2}} d r^{2}+r^{2} d \theta^{2}+r^{2} \sin ^{2} \theta d \phi^{2}\right)
$$

where $a(t)$ characterizes the expansion (or contraction) of the Universe and is usually called the scale factor. $(r, \theta, \phi)$ are the radial distance, azimuthal angle, and polar

\footnotetext{
${ }_{1}^{1}$ http://wigglez.swin.edu.au/site/

${ }^{2}$ http://www.sdss3.org/surveys/boss.php
} 
angle. In this dissertation, the speed of light $c$ is set to 1 and the metric signature is taken to be $(-1,1,1,1)$, unless stated otherwise. Depending on the geometry of the Universe, the spatial curvature parameter $k$ can take different values: -1 (for an open universe), 0 (for a spatial flat universe), 1 (for a closed universe). Furthermore, we assume the matter in the Universe can be treated as perfect fluid with homogeneous density $\rho$ and pressure $p$. The energy-momentum tensor can then be written as

$$
T_{\mu \nu}=(\rho+p) U_{\mu} U_{\nu}+p g_{\mu \nu}
$$

where $U_{\mu}$ is the four-velocity of the fluid, which satisfies $U^{\mu} U_{\mu}=-1$. In the coordinate system comoving with the fluid, $U_{\mu}=(-1,0,0,0)$. Substituting the FLRW metric Eq. (2.1) and the energy-momentum tensor Eq. (2.2) into Einstein equations

$$
R_{\mu \nu}-\frac{1}{2} g_{\mu \nu} R+g_{\mu \nu} \Lambda=8 \pi G T_{\mu \nu}
$$

we obtain the Friedmann equations that govern the evolution of $a$ :

$$
\begin{aligned}
H^{2} & =\frac{8 \pi G}{3} \rho+\frac{\Lambda}{3}-\frac{k}{a^{2}}, \\
\ddot{a} & =-\frac{4 \pi G}{3}(\rho+3 p)+\frac{\Lambda}{3} .
\end{aligned}
$$

Here $R_{\mu \nu}$ and $R$ are Ricci curvature tensor and scalar curvature, respectively. The dot ،. ' denotes derivatives with respect to cosmic time. $H \equiv \frac{\dot{a}}{a}$ is the Hubble parameter, which measures the expansion rate of the Universe. $G$ is the gravitational constant. We have also included a cosmological constant $\Lambda$. Since the spatial curvature of the Universe is found to be very close to zero [18, 77, 19], we will set $k=0$ in the following discussions. Another useful equation is the continuity equation, which can be derived from the conservation law $\left(\nabla_{\mu} T^{\mu \nu}=0\right)$,

$$
\dot{\rho}+3 H(\rho+p)=0 .
$$

Given the state parameter of the matter content, $w=p / \rho$, we can solve Eq. 2.4 and Eq. (2.6) [or Eq. (2.5)] to get the evolution of $a$.

(1) Radiation and relativistic matter $\left(w=\frac{1}{3}\right)$

$$
\rho \propto a^{-4}, \quad a \propto t^{1 / 2} .
$$

(2) Non-relativistic matter $(w \approx 0)$

$$
\rho \propto a^{-3}, \quad a \propto t^{2 / 3} .
$$


(3) Cosmological constant $(w=-1)$

$$
\rho=\frac{\Lambda}{8 \pi G}, \quad a \propto e^{\sqrt{\frac{\Lambda}{3}} t} .
$$

From Eq. (2.5), we can see that the expansion of the Universe will slow down if the Universe is composed of only radiation and matter since they both have non-negative state parameters. But as is well known, in 1998 the High-Z Supernova Search Team and the Supernova Cosmology Project discovered, independently of each other, that the expansion of the Universe is actually accelerating [14, 15]. Therefore, a non-zero cosmological constant which has $w=-1$ is a necessary component.

Now we consider the evolution of a classical scalar field $\varphi$ which describes the axion dark matter in a homogeneous and isotropic universe. The Lagrangian of $\varphi$ takes the form of

$$
\mathcal{L}_{\varphi}=-\frac{1}{2} \partial_{\mu} \varphi \partial^{\mu} \varphi-V(\varphi)
$$

For FDM, we can take $V(\varphi)=\frac{1}{2} m_{a}^{2} \varphi^{2}$, i.e. neglecting all the higher order selfinteractions. Note that $\partial^{\mu} \varphi \equiv g^{\mu \nu} \nabla_{\nu} \varphi=g^{\mu \nu} \partial_{\nu} \varphi$ with $\nabla_{\nu}$ denoting the covariant derivative. The equation of motion (EoM) of $\varphi$ is then given by

$$
\left(\square-m_{a}^{2}\right) \varphi=0
$$

where $\square=\frac{1}{\sqrt{-g}} \partial_{\mu}\left(\sqrt{-g} g^{\mu \nu} \partial_{\nu}\right)$ is the d'Alembert operator. For homogeneous $\varphi$, Eq. (2.11) is simplified to

$$
\ddot{\varphi}+3 H \dot{\varphi}+m_{a}^{2} \varphi^{2}=0 .
$$

It is exactly the same as the equation of motion for a harmonic oscillator with an additional friction term (the Hubble friction). Thus the oscillation of $\varphi$ will be damped by the expansion of the Universe and depending on the damping ratio $\zeta=\frac{2 H}{3 m_{a}}, \varphi$ will behave very differently.

Assume that at the Peccei-Quinn phase transition [41, the axion field gets an initial displacement $\varphi_{i}$. Since at this time the Hubble rate is much larger than the axion mass, the damping ratio $\zeta \gg 1$ and the oscillation of $\varphi$ is over-damped. Thus $\varphi$ will roll down the potential hill very slowly (similar to the slow-roll inflation scenario) and act as one component of dark energy.

As the Universe expands, the Hubble rate decreases, so does the damping ratio $\zeta$. As $\zeta$ drop below 1 , the critical value, $\varphi$ starts to oscillate but with a decreasing amplitude (see the left plot of Fig. 2.1). In the oscillation phase, the scalar field with a quadratic potential as we consider here has an effective state parameter of 0 [49], i.e. it behaves as dark matter. 

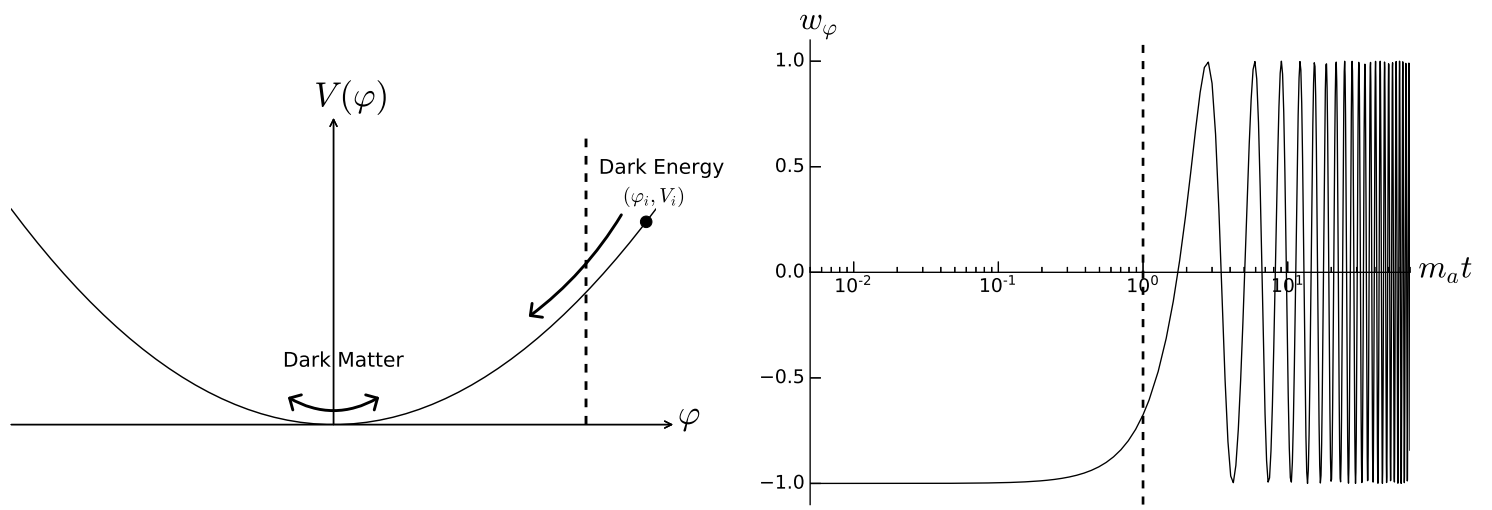

Figure 2.1: Evolution of the scalar field in a homogeneous universe. Left: potential of the scalar field. The solid curve shows the approximated axion potential up to the quadratic term. The vertical dashed line marks the time when axion begins oscillating and behaves as dark matter. Right: state parameter of the scalar field.

The transition from dark energy to dark matter can happen in different epochs of the Universe depending on the axion mass. For $m_{a} \gtrsim 10^{-27} \mathrm{eV}$, the scalar field start oscillating before matter-radiation equality thus could be a possible dark matter candidate, while for $m_{a} \lesssim 10^{-33} \mathrm{eV}$, it behaves always as dark energy till the present time (see e.g. [66]). In the thesis, I will concentrate on FDM with $m_{a}$ in the range $10^{-22}-10^{-20} \mathrm{eV}$, for which the oscillation starts well before matter-radiation equality, so we can safely treat the expansion of the Universe the same as in the $\Lambda$ CDM model.

More precisely, if we take $a(t) \propto t^{\gamma}$, Eq. 2.12 can be solved analytically and the solution of $\varphi$ is given by [78]

$$
\varphi=a(t)^{-3 / 2}\left(m_{a} t\right)^{1 / 2}\left(A J_{n}\left(m_{a} t\right)+B Y_{n}\left(m_{a} t\right)\right),
$$

where $J_{n}$ and $Y_{n}$ are Bessel functions of the first kind and second kind, respectively, and $n=(3 \gamma-1) / 2$. The integration constants $A$ and $B$ are set by initial conditions: $\varphi\left(t_{i}\right)=\varphi_{i}, \dot{\varphi}\left(t_{i}\right)=0$. In the radiation-dominated era, $\gamma=1 / 2$. In the matterdominated era, $\gamma=2 / 3$.

From the Lagrangian Eq. 2.10), we can obtain the energy-momentum tensor of the scalar field

$$
T_{\mu \nu}=\partial_{\mu} \varphi \partial_{\nu} \varphi+g_{\mu \nu} \mathcal{L}_{\varphi}
$$

Comparing it with the energy-momentum tensor of perfect fluid Eq. (2.2) and applying the normalization condition $U^{\mu} U_{\mu}=-1$, we can change to the fluid description 
of the scalar field

$$
\begin{aligned}
\rho_{\varphi} & =-\frac{1}{2} \partial^{\mu} \varphi \partial_{\mu} \varphi+\frac{1}{2} m_{a}^{2} \varphi^{2} \\
p_{\varphi} & =-\frac{1}{2} \partial^{\mu} \varphi \partial_{\mu} \varphi-\frac{1}{2} m_{a}^{2} \varphi^{2} \\
U_{\mu} & =\frac{\partial_{\mu} \varphi}{\sqrt{-\partial^{\alpha} \varphi \partial_{\alpha} \varphi}} .
\end{aligned}
$$

For a homogeneous and isotropic scalar field, the energy density $\rho_{\varphi}$ and pressure $p_{\varphi}$ are simplified to

$$
\begin{aligned}
\rho_{\varphi} & =\frac{1}{2} \dot{\varphi}^{2}+\frac{1}{2} m_{a}^{2} \varphi^{2}, \\
p_{\varphi} & =\frac{1}{2} \dot{\varphi}^{2}-\frac{1}{2} m_{a}^{2} \varphi^{2} .
\end{aligned}
$$

Substituting the solution of $\varphi$, Eq. (2.13), into Eqs. 2.18) and (2.19), we can compute the state parameter $w_{\varphi}=p_{\varphi} / \rho_{\varphi}$. The right plot of Fig. 2.1 shows $w_{\varphi}$ with respect to cosmic time. As can be seen, at early times $w_{\varphi} \sim-1$, thus $\varphi$ behaves as dark energy. With the expansion of the Universe, $w_{\varphi}$ increases gradually and finally starts oscillating when $t \sim H^{-1} \gtrsim m_{a}^{-1}$. The period of the oscillation $\Delta t_{\mathrm{osc}}=m_{a}^{-1}$. For $m_{a}>10^{-22} \mathrm{eV}$ as we consider in this dissertation, the period is extremely small compared to the Hubble time: $\Delta t_{\text {osc }}<10^{-11} H_{0}^{-1}$. Therefore, when considering the background evolution, we can take the average of quantities related to $\varphi$, such as $\rho_{\varphi}$ and $p_{\varphi}$, over the oscillation time scale and neglect the effects on time scales shorter than $\Delta t_{\text {osc }}$.

When $H / m_{a} \ll 1\left(m_{a} t \gg 1\right)$, the solution Eq. (2.13) can be approximated by [79]

$$
\varphi=a(t)^{-3 / 2}\left[\varphi_{+} \sin \left(m_{a} t\right)+\varphi_{-} \cos \left(m_{a} t\right)\right]
$$

where $\varphi_{+}$and $\varphi_{-}$are constants. Substituting the approximated solution into the expressions of energy density and pressure, and taking averages over the time scale $m_{a}^{-1}$, we have

$$
\begin{aligned}
\left\langle\rho_{\varphi}\right\rangle & =\frac{1}{2} m_{a}^{2} a^{-3}\left(\varphi_{+}^{2}+\varphi_{-}^{2}\right) \\
\left\langle p_{\varphi}\right\rangle & =0 .
\end{aligned}
$$

Here $\langle$.$\rangle denotes averaging over \Delta t_{\text {osc }}$. The effective average state parameter $\left\langle w_{\varphi}\right\rangle=0$, so the scalar field behaves as dark matter. 


\subsubsection{Linear Perturbations}

In the last subsection, the Universe is treated as homogeneous and isotropic. Now let us consider small perturbations on this homogeneous background. In the Newtonian gauge and assuming vanishing anisotropic stress, the perturbed FRW metric can be written as

$$
d s^{2}=-(1+2 \Phi) d t^{2}+a(t)^{2}(1-2 \Phi) \delta_{i j} d x^{i} d x
$$

where $\Phi$ is the gravitational potential and $\delta_{i j}$ is the Kronecker delta function. Here we are only interested in the scalar perturbations since they are most relevant to cosmic structure formation. Accordingly, the perturbed perfect fluid energy-momentum tensor is

$$
\begin{aligned}
T_{00} & =\bar{\rho}(1+2 \Phi)+\delta \rho, \\
T_{0 i} & =-(\bar{\rho}+\bar{p}) \partial_{i} \delta u, \\
T_{i j} & =a^{2} \delta_{i j}[\bar{p}(1-2 \Phi)+\delta p] .
\end{aligned}
$$

Here the quantities with overlines refer to the background values. $\delta u$ is defined in such a way that the longitudinal part of the spatial velocity perturbation $\delta U_{i}^{L}=$ $\partial_{i} \delta u$. Substituting the perturbed metric and energy-momentum tensor into Einstein equations, Eq. (2.3), and the continuity equation, Eq. (2.6), and keeping only the firstorder terms, we get the equations that govern the evolution of linear perturbations. The derivation can be found in many textbooks on cosmology, e.g. [80]. I will not repeat the details here, but just list some of the most important equations that will be useful for later discussions. For simplicity, we can write the equations in Fourier space. In the linear regime, there are no couplings between different Fourier modes. For example, performing a Fourier transform of the density perturbation, we have

$$
\delta \rho(\mathbf{x}, t)=\int d^{3} k \delta \rho_{k}(\mathbf{k}, t) e^{i \mathbf{k} \cdot \mathbf{x}},
$$

where $\mathbf{k}$ is the comoving wavenumber.

In the matter-dominated era, we can take $\bar{p}=0$. But in general, the pressure perturbation $\delta p$ is not necessarily 0 . In the sub-horizon regime $(k / a \gg H)$, the linear density perturbation satisfies

$$
\ddot{\delta}+2 H \dot{\delta}-\left(4 \pi G \bar{\rho}-\frac{k^{2}}{a^{2}} c_{s}^{2}\right) \delta=0,
$$

where $\delta=\delta \rho / \bar{\rho}$ is the overdensity and

$$
c_{s} \equiv \sqrt{\frac{\delta p}{\delta \rho}}
$$


is the sound speed.

For pressureless CDM, $c_{s}=0$. Recalling that in the matter-dominated era, $\bar{\rho} \propto$ $a^{-3}$ and $a \propto t^{2 / 3}$ [see Eq. 2.8] ], Eq. 2.28) can be solved analytically. $\delta$ has the following solution

$$
\delta_{\mathrm{CDM}}=C_{1} a+C_{2} a^{-3 / 2},
$$

where $C_{1}$ and $C_{2}$ are constants fixed by initial conditions. As can be seen, the solution of $\delta$ contains one growing mode $(\propto a)$ and one decaying mode $\left(\propto a^{-3 / 2}\right)$. Both modes are expected to be very small in the early Universe. As the Universe expands the decaying mode will becomes negligible, thus we will only be concerned with the growing mode. The growth of $\delta_{\mathrm{CDM}}$ is independent of wavenumber 3 , so the formation of large scale structure will start on all scales. As will be shown later, it leads to the formation of a large amount of small dark matter halos.

For FDM, it is a bit more complicated. The perturbation $\delta \varphi$ oscillates rapidly just as the background field which makes it difficult to solve even numerically. There are several ways to deal with this problem [81]. One way is to use a similar ansatz for the oscillation of linear perturbations as for the background $\bar{\varphi}$ (see Eq. (2.20)) and compute the perturbations in the fluid description by taking average over the time scale $m^{-1}$. This is the common strategy used in previous literature, e.g. [78, 82, 83, 66, 84]. In [81], a different approach based on field transformation is proposed. Here I will follow the former approach and take the ansatz

$$
\delta \varphi=\delta \varphi_{+} \sin \left(m_{a} t\right)+\delta \varphi_{-} \cos \left(m_{a} t\right) .
$$

Taking $\varphi=\bar{\varphi}+\delta \varphi$, we can compute the linear perturbations of the density, pressure in the fluid description from Eqs. (2.15)-(2.17):

$$
\begin{aligned}
& \delta \rho_{\varphi}=\dot{\bar{\varphi}} \dot{\delta \varphi}+m_{a}^{2} \bar{\varphi} \delta \varphi-\Phi(\dot{\bar{\varphi}})^{2} \\
& \delta p_{\varphi}=\dot{\bar{\varphi}} \dot{\delta \varphi}-m_{a}^{2} \bar{\varphi} \delta \varphi-\Phi(\dot{\bar{\varphi}})^{2} .
\end{aligned}
$$

Furthermore, the perturbed EoM of $\varphi$, Eq. (2.11), can be written as

$$
\ddot{\delta} \varphi+3 H \dot{\delta \varphi}+\left(\frac{k^{2}}{a^{2}}+m_{a}^{2} a^{2}\right) \delta \varphi=4 \dot{\bar{\varphi}} \dot{\Phi}-2 m_{a}^{2} \bar{\varphi} \Phi
$$

Substituting the ansatz Eq. 2.20) and Eq. 2.31) into Eq. 2.34 and keeping only leading-order terms with respect to $H / m_{a}$, we obtain

$$
\Phi=-\frac{k^{2}}{2 m_{a}^{2} a^{2}} \frac{\delta \varphi}{\bar{\varphi}} .
$$

\footnotetext{
${ }^{3}$ Actually, if we look at the perturbations on scales $k / a \rightarrow H$, the growth will behave differently. But on scales of galaxies and galaxy clusters (several hundred kpc to several Mpc), it is reasonably accurate to treat the growth of $\delta_{\mathrm{CDM}}$ as scale-independent.
} 
The density and pressure perturbations averaged over the time scale $m^{-1}$ are then given by

$$
\begin{aligned}
\left\langle\delta \rho_{\varphi}\right\rangle & =\left(1+\frac{k^{2}}{4 m_{a}^{2} a^{2}}\right) a^{-3 / 2} m_{a}^{2}\left(\varphi_{+} \delta \varphi_{+}+\varphi_{-} \delta \varphi_{-}\right) \\
\left\langle\delta p_{\varphi}\right\rangle & =\frac{k^{2}}{4 m_{a}^{2} a^{2}} a^{-3 / 2} m_{a}^{2}\left(\varphi_{+} \delta \varphi_{+}+\varphi_{-} \delta \varphi_{-}\right) .
\end{aligned}
$$

With the average density and pressure perturbations, we can calculate the effective sound speed

$$
c_{s, \mathrm{eff}}^{2}=\frac{\left\langle\delta p_{\varphi}\right\rangle}{\left\langle\delta \rho_{\varphi}\right\rangle}=\frac{k^{2}}{4 m_{a}^{2} a^{2}} \frac{1}{1+\frac{k^{2}}{4 m_{a}^{2} a^{2}}} .
$$

As mentioned in [40], $c_{s, \text { eff }}$ is not gauge invariant. But the difference between different gauges, e.g. the Newtonian gauge and the synchronous gauge, becomes negligible in the sub-horizon regime. Particularly, in the gauge comoving with the time-averaged FDM fluid, Eq. 2.38) is valid on all scales [82, 83].

Substituting the expression of effective sound speed into Eq. 2.28), we have

$$
\ddot{\delta}_{a}+2 H \dot{\delta_{a}}-\left(4 \pi G \rho_{a}-\frac{k^{2}}{a^{2}} \frac{k^{2}}{4 m_{a}^{2} a^{2}} \frac{1}{1+\frac{k^{2}}{4 m_{a}^{2} a^{2}}}\right) \delta_{a}=0,
$$

where $\delta_{a}=\delta \rho_{\varphi} / \overline{\rho_{\varphi}}$.

In the limit $k / a \ll 2 m_{a}$, i.e. on scales larger than the Compton wavelength of FDM particles, the effective sound speed $c_{s, \text { eff }} \sim \frac{k^{2}}{4 m_{a}^{2} a^{2}}$. If $k / a \ll 1, c_{s, \text { eff }} \sim 0, \delta_{a}$ will grow just as in the CDM model. But if $c_{s, \text { eff }}$ is non-negligible, the pressure of FDM fluid will counteract the gravity, thus lead to a suppression in the growth of $\delta_{a}$. More specifically, $\delta_{a}$ will not grow below the comoving Jeans scale [85, 25]

$$
k_{J}=\left(16 \pi G \bar{\rho}_{\varphi}\right)^{\frac{1}{4}} m_{a}^{\frac{1}{2}} a .
$$

Here we have assumed a universe dominated by FDM. Accordingly, we can define the Jeans mass

$$
M_{J}=\frac{4}{3} \pi\left(a \frac{\pi}{k_{J}}\right)^{3} \bar{\rho}_{\varphi} .
$$

Unlike in the CDM model which predicts the formation of a lot of small dark matter halos, in FDM model the number of halos with mass smaller than $M_{J}$ is largely suppressed. 


\subsubsection{Nonlinear Evolution in the Nonrelativistic Limit}

In the last subsection, we considered the evolution of linear perturbations. To study nonlinear clustering, e.g. halo formation, we need a nonlinear treatment of the EoM for FDM. In most cases relevant to cosmic structure formation considered in this dissertation, FDM can be treated in the nonrelativistic limit 4 . In this limit, the Newtonian potential $\Phi$ is small, so the perturbed FRW metric, Eq. 2.23), is still valid. But instead of directly solving the Klein-Gordon equation, Eq. (2.11), it is usually useful to write the scalar field $\varphi$ as

$$
\varphi=\sqrt{\frac{\hbar^{3}}{2 m_{a}}}\left(\psi e^{-i m_{a} t / \hbar}+\psi^{*} e^{i m_{a} t / \hbar}\right),
$$

where $\psi$ is a complex scalar field. Substituting Eqs. (2.42) and (2.23) into Eq. (2.11) and assuming $|\ddot{\psi}| \ll m|\dot{\psi}| / \hbar$, i.e. the amplitude of $\varphi$ varies relatively slowly with time compared to the fast oscillation of $\varphi$, we get a Schrödinger-like equation

$$
i \hbar\left(\dot{\psi}+\frac{3}{2} H \psi\right)=\left(-\frac{\hbar^{2}}{2 m_{a} a^{2}} \nabla^{2}+m_{a} \Phi\right) \psi .
$$

Here we have neglected higher-order terms such as $\Phi \dot{\psi}, \Phi \nabla^{2} \psi$, and $\dot{\Phi} \dot{\psi}$ (see [40] for more details). The Newtonian potential $\Phi$ satisfies the Poisson equation

$$
\frac{1}{a^{2}} \nabla^{2} \Phi=4 \pi G m_{a}|\psi|^{2}
$$

where we have used the approximation that the energy density of $\rho \approx|\psi|^{2}$ in the nonrelativistic limit.

As is done for linear perturbations, we can also write Eq. 2.43) in the fluid description. Defining

$$
\begin{aligned}
\psi & \equiv \sqrt{\frac{\rho}{m}} e^{i \theta}, \\
\mathbf{v} & \equiv \frac{\hbar}{m_{a} a} \nabla \theta,
\end{aligned}
$$

Eq. 2.43) can be transformed to the Madelung equations [86]

$$
\begin{aligned}
\dot{\rho}+3 H \rho+\frac{1}{a} \nabla \cdot(\rho \mathbf{v}) & =0 \\
\dot{\mathbf{v}}+H \mathbf{v}+\frac{1}{a}(\mathbf{v} \cdot \nabla) \mathbf{v} & =-\frac{1}{a} \nabla \Phi+\frac{\hbar^{2}}{2 m_{a}^{2} a^{3}} \nabla\left(\frac{\nabla^{2} \sqrt{\rho}}{\sqrt{\rho}}\right) .
\end{aligned}
$$

\footnotetext{
${ }^{4}$ Note that the virial velocity of a typical dark matter halo is $\sim 100 \mathrm{~km} / \mathrm{s}$ which is much smaller than the speed of light. Black hole formation from FDM is beyond the scope of this dissertation.
} 
Comparing the second equation with the classical Euler equations, we can find that there appears an extra pressure term

$$
P_{Q} \equiv-\frac{\hbar^{2}}{2 m_{a}^{2} a^{3}}\left(\frac{\nabla^{2} \sqrt{\rho}}{\sqrt{\rho}}\right)
$$

which is often called quantum pressure.

\subsubsection{Spherical Collapse Model}

In Section 2.1.2, we considered the evolution of linear perturbations analytically. But when perturbations of the density field become highly nonlinear, numerical simulations are usually needed. For FDM, we will need to solve either the SchrödingerPoisson equations or the Madelung-Poisson equations as shown in Section 2.1.3. However, we will first consider an idealized case, i.e. spherical collapse, which can be solved analytically for CDM. This example is commonly used in the standard textbooks, e.g. [87, 88, to demonstrate the halo formation. Although it may be oversimplified to some extent, it has some very important results that are widely used in semi-analytic models of structure formation.

The Universe is assumed to be matter-dominated $\left(a \propto t^{2 / 3}\right)$. The background matter density is

$$
\rho_{m}(t)=\frac{\rho_{m}(0)}{a(t)^{3}}=\frac{1}{6 \pi G t^{2}} .
$$

Now consider a uniform sphere with an initial overdensity of

$$
\delta_{i}=\frac{\rho\left(t_{i}\right)-\rho_{m}\left(t_{i}\right)}{\rho_{m}\left(t_{i}\right)},
$$

and a initial radius of $r_{i}$. Here we require that $\delta_{i} \ll 1$ so that at the initial time the overdensity is still in the linear regime. We will first consider the CDM model, i.e. the pressure is negligible. The total mass contained in the sphere

$$
M=\frac{4}{3} \pi r_{i}^{3}\left(1+\delta_{i}\right) \rho_{m}\left(t_{i}\right)
$$

The change of the sphere's radius is described by Newtonian dynamics

$$
\frac{d^{2} r}{d t^{2}}=-\frac{G M}{r^{2}} .
$$

A parametric solution to Eq. 2.53 can be found:

$$
\begin{aligned}
r(\theta) & =A(1-\cos \theta), \\
t(\theta) & =B(\theta-\sin \theta),
\end{aligned}
$$



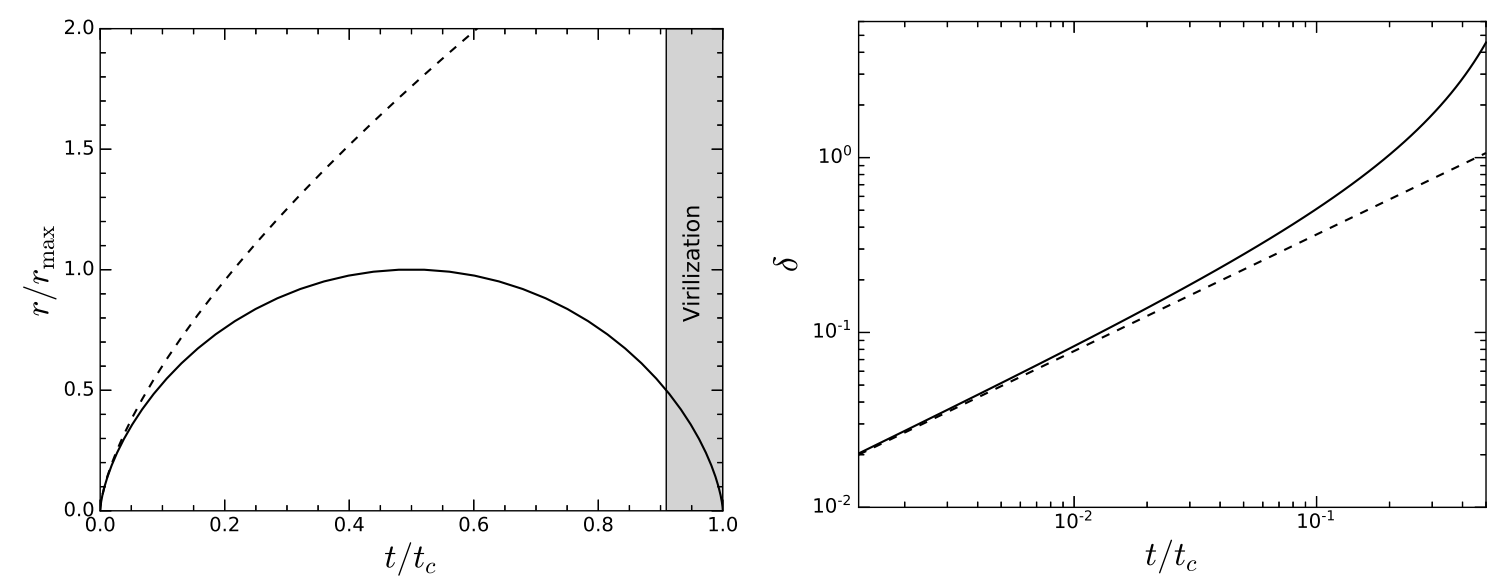

Figure 2.2: Spherical collapse. Left: evolution of the radius of the overdense sphere (solid line) compared to the background expansion (dashed line). In the realistic case, the sphere will not collapse to a singularity but virialize via phase mixing and violent relaxation (indicated by the shaded region). Right: evolution of the overdensity $\delta$ (solid line) compared to the prediction from the linear perturbation theory $\delta_{\text {lin }}$. At the beginning, $\delta$ is small and grows just like $\delta_{\text {lin }}$. As $\delta$ increases, it enters the nonlinear regime and grows faster than $\delta_{\text {lin }}$. At the time $t_{m}$ when the radius of the overdense sphere reaches its maximum, $\delta \approx 4.28 \delta_{\operatorname{lin}}$.

where $\frac{A^{3}}{B^{2}}=G M$. The parameters $A$ and $B$ are fixed by initial conditions. At the initial time, $\theta \ll 1$, so we can expand Eq. 2.54 as $r_{i}=\frac{1}{2} A \theta_{i}^{2}+O\left(\theta_{i}^{4}\right)$. Similarly, substituting Eq. (2.54) and Eq. 2.55) into Eq. 2.52 and expanding to $O\left(\theta^{4}\right)$, we can get $\delta_{i}=\frac{3}{20} \theta_{i}^{2}+O\left(\theta_{i}^{4}\right)$. Thus, the parameters $A$ and $B$ can be expressed as

$$
\begin{aligned}
A & =\frac{3}{10} \frac{r_{i}}{\delta_{i}}=\frac{3}{10}\left(\frac{9}{2} G M\right)^{1 / 3} \frac{t_{i}^{2 / 3}}{\delta_{i}}, \\
B & =\sqrt{\frac{A^{3}}{G M}}=\frac{9}{20} \sqrt{\frac{3}{5}} \frac{t_{i}}{\delta_{i}^{3 / 2}} .
\end{aligned}
$$

Noting that $t \approx \theta^{3}$ when $\theta \ll 1$, we have $\delta \propto t^{2 / 3} \propto a(t)$ at early times, which is exactly the same as the prediction from the linear perturbation theory (see the right panel of Fig. 2.2).

From Eq. (2.54) and Eq. 2.55), we can see that the radius of the spherical overdensity has a maximum of $2 A$ when $\theta=\pi$. The corresponding time is $t_{m}=B \pi$. After $t_{m}$, the sphere will stop expanding and start to collapse (see the left panel of Fig. 2.2). In the ideal case, the sphere will collapse to a singularity at $t=2 t_{m}$. It indicates that the spherical collapse model failed at this time. In the realistic case, the collapsing sphere will relax to a virialized halo. In practice, it is usually assumed that at $t_{c}=2 t_{m}$, i.e. when the sphere collapse to a singularity in the idealized model, 
the halo is virialized. We will call $t_{c}$ the collapse time which can be computed as

$$
t_{c}=2 B \pi=\frac{9}{10} \sqrt{\frac{3}{5}} \pi \frac{t_{i}}{\delta_{i}^{3 / 2}} .
$$

From Eq. 2.58), we can see that the larger the initial overdensity, the earlier it will collapse to a virialized halo. There exists a critical value

$$
\delta_{i}^{c}=\frac{3}{5}\left(\frac{3 \pi}{2}\right)^{2 / 3}\left(\frac{t_{i}}{t_{c}}\right)^{2 / 3} \approx 1.686\left(\frac{t_{i}}{t_{c}}\right)^{2 / 3},
$$

so that if $\delta_{i}>\delta_{i}^{c}$, the halo will collapse before $t_{c}$.

An important implication of Eq. (2.59) is that it tells us we can identify a halo far before nonlinear structure formation begins by investigating the linear overdensity. It enables us to predict the halo abundance from the linear density perturbations, which is the basis of the Press-Schechter theory [89] that will be discussed in the next subsection.

As mentioned before, in the realistic case, the sphere will collapse and finally form a virialized halo. Now let us estimate the average density of a virialized halo. Assume that all shells in the sphere reach the maximum radius and turn around at the same time $t=t_{m}$. At $t_{m}$, the kinetic energy of the system $E_{k}=0$ and the total energy $E=U\left(r_{\max }\right)$. Here $r_{\max }=2 A$ is the maximum radius of the sphere. According to the virial theorem, when the halo is virialized, $E_{k}=-\frac{1}{2} U\left(r_{\text {vir }}\right)$. Thus the total energy $E=\frac{1}{2} U\left(r_{\text {vir }}\right)=U\left(r_{\max }\right)$. Since the potential energy is proportional to $1 / r$, we have $r_{v i r}=\frac{1}{2} r_{\max }=A$. The density contrast at virialization is then given by

$$
\Delta_{\mathrm{vir}}=\frac{M}{\frac{4}{3} \pi A^{3} \rho_{m}\left(t_{c}\right)}=18 \pi^{2} \approx 178 .
$$

Here we have used Eq. 2.50 and the relation $\frac{A^{3}}{B^{2}}=G M$. Eq. 2.60 is usually used to define a virialized halo in cosmological simulations.

For FDM, there are two main differences:

(1) as shown in Section 2.1.2, linear overdensities with a comoving wavenumber $k$ do not grow until the Jeans wavenumber $k_{J} \propto a^{1 / 4}$ becomes larger than $k$. Thus collapsing starts later in FDM model, which is most significant for small halos. In the extreme case, on scales below the current Jeans scale collapsing does not happen at all.

(2) the FDM fluid is not pressureless. The quantum pressure will stop the sphere from further collapsing when it balances the gravity force. Thus we would 
expect a smoother density profile in the central region of a collapsed halo. The virial theorem still holds for virialized halos, i.e. $E_{k}=-\frac{1}{2} U$, but the kinetic energy becomes $E_{k}=E_{k}^{\text {classic }}+E_{Q}$ where $E_{k}^{\text {classic }}$ is the classic kinetic energy and $E_{Q}$ corresponds to the quantum pressure and is usually called "gradient energy" or "quantum kinetic energy".

To show the possible differences between CDM and FDM, we simulate spherical collapse in FDM model with a one-dimensional pseudo-spectral code. The mass of FDM particle is taken to be $10^{-22} \mathrm{eV}$. The techniques will be discussed in detail later. Although such a simulation is limited by the assumption of spherical symmetry compared to a full three-dimensional cosmological simulation, it can be useful for finding the critical overdensity for collapse in dark matter models other than CDM. For example, [90] simulate the spherical collapse of warm dark matter (WDM) halos using a one-dimensional hydrodynamics code. With the critical overdensity for collapse found in [90], [91] compute the halo mass function in the WDM model and show that it is consistent with the result from N-body cosmological simulation.

We assume a matter-dominated universe and the initial overdensity is taken to be a single spherical Fourier mode

$$
\delta\left(x, t_{i}\right)=\delta_{i} \frac{\sin \left(k_{s} x\right)}{k_{s} x} .
$$

Here $x=r / a$ is the comoving distance to the origin and $k_{s}$ characterizes the scale of the overdense region. The initial peculiar velocity field is created by solving the linear perturbed continuity equation

$$
\nabla \cdot \mathbf{v}\left(r, t_{i}\right)=-\dot{\delta}\left(r, t_{i}\right)
$$

We start the simulation at $z=1000$ so that the overdensity is still in the linear regime at the beginning. As shown in Section 2.1.2, for CDM the linear overdensity $\delta$ grows as $a$. For FDM, the growth will depend on the wavenumber. For simplicity we have adopted the same condition $\dot{\delta} \propto \dot{a} \propto t^{-1 / 3}$ to different $k_{s}$. But since we start the simulation at very high redshift, we expect that the artificial growth will be largely damped at later time.

Fig. 2.3 shows the results from two typical simulations with $k_{s}=0.5 k_{J, 1000}$ and $k_{s}=k_{J, 1000}$, respectively. Here $k_{J, 1000}$ is the comoving Jeans wavenumber (see Eq. (2.40) ) at $z=1000$. The initial amplitude of the overdensity $\delta_{i}$ is set to 0.02 . The left panel shows the density profiles at the time when the halos form. Prominent cores can be seen in both cases and their profiles can be well fitted by the solitonic 

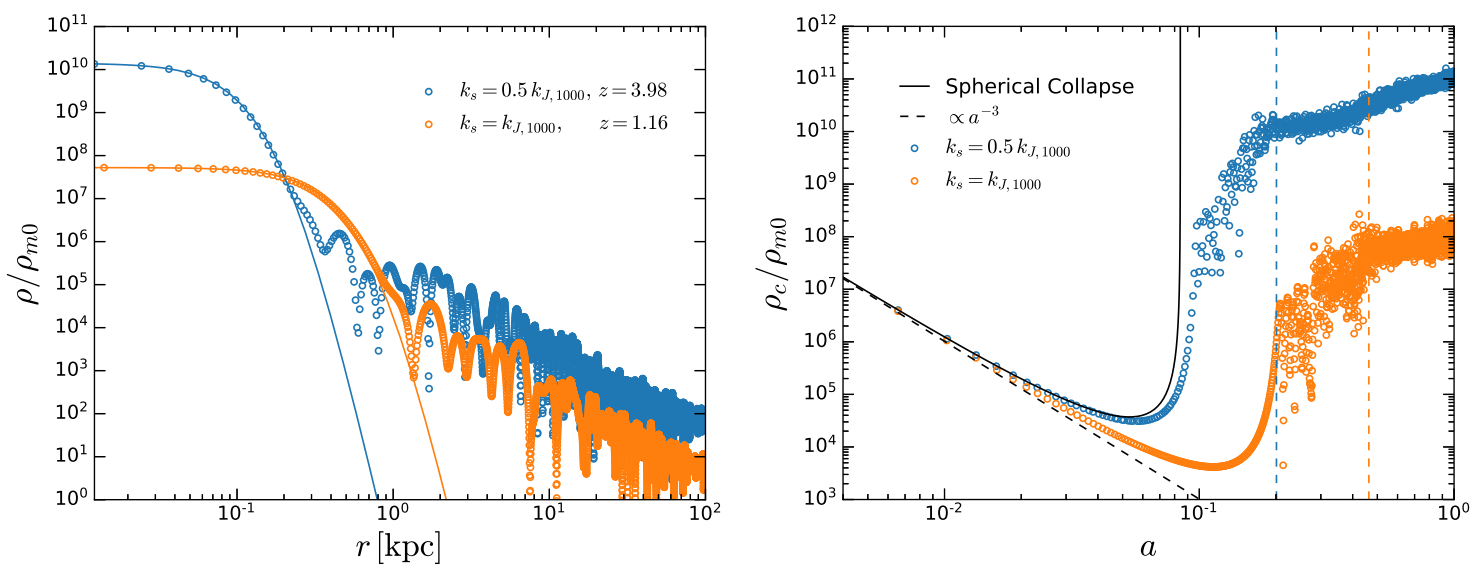

Figure 2.3: Simulations of spherical collapse with FDM. Left: density profile at the collapse time for different initial overdensities. $\rho_{m 0}$ is the background matter density at current time. The initial overdensity is taken to be a single spherical Fourier mode, i.e. $\delta\left(x, t_{i}\right)=\delta_{i} \sin \left(k_{s} x\right) /\left(k_{s} x\right)$. Two simulations have the same $\delta_{i}=0.02$ but different $k_{s}$. Right: evolution of the central density $\rho_{c}$. The collapse time is marked by the vertical lines.

profiles (solid lines) [29, 4, 31] (see also Eq. (2.104)). In the outer region, the density profile drops approximately as $r^{-\alpha}$ with $\alpha \sim 2-35^{5}$. The right panel shows the evolution of the central density $\rho_{c}$. At early times, $\rho_{c}$ decreases as $a^{-3}$ (black dashed line) due to the expansion of the Universe. As the overdensity grows, the expansion of the overdense region slows down compared to the background. Finally, the sphere starts to collapse and $\rho_{c}$ increases rapidly as in the simple spherical collapse model (black solid line). At late times, the overdense sphere virializes and a core forms in the center (indicated by the dashed vertical lines). After the formation of the core, $\rho_{c}$ increases only mildly, which has also been shown in 3-D cosmological simulation 92. We define the collapse time as the time the core appears to have a soliton profile. Under this definition, the initial overdensity with $k_{s}=0.5 k_{J, 1000}$ and $\delta_{i}=0.02$ collapse at $z=3.98$. With a larger initial wavenumber $k_{s}=k_{J, 1000}$, the overdensity collapses later, at $z=1.16$. Although more careful analysis is needed to get the critical overdensity for collapse for different $k$, our simulations have demonstrated the two main differences between FDM and CDM as we discussed before: (1) FDM halos collapse later; (2) FDM halos have smoother density profiles in the central region.

\footnotetext{
${ }^{5}$ We have assumed spherical symmetry, so the outer region does not necessarily have a NFW-like profile as found in 3-D simulations of FDM, e.g. [29, 4, 62, 63, 92.
} 


\subsubsection{Press-Schechter Formalism}

As is shown in the previous subsection, for CDM if the overdensity is larger than $\delta_{i}^{c}$ at $t_{i}$, it will collapse before $t_{c}$. The critical overdensity $\delta_{i}^{c}$ is give by Eq. (2.59). If we extrapolate the overdensity to the current time by the linear growth factor $D(t) \equiv \delta(t) / \delta\left(t_{0}\right)$, even though $\delta$ may have already become much larger than 1 , we can obtain the current value of the overdensity that has a critical value at $t_{i}$ predicted by the linear perturbation theory

$$
\delta_{c}\left(t_{c}\right) \equiv \frac{\delta_{i}^{c}}{D\left(t_{i}\right)}=\frac{1.686}{D\left(t_{c}\right)}
$$

Here we have used the fact that in the matter-dominated universe, the linear overdensity grows as $\delta \propto t^{2 / 3}$. Thus if the linear extrapolated overdensity at the current time is larger than $\delta_{c}\left(t_{c}\right)$, it implies that the corresponding mass should have collapsed before $t_{c}$.

In the Press-Schechter theory [89], to connect the overdense regions to collapsed halos with certain masses, a filter is adopted which smooths out the overdensities on scales smaller than the filter scale. Given a smoothing function, or as it is usually called a window function, $W(\mathbf{x}, R)$, the smoothed overdensity is obtained by performing a convolution:

$$
\delta_{R}(\mathbf{x})=\int \delta\left(\mathbf{x}^{\prime}\right) W\left(\mathbf{x}-\mathbf{x}^{\prime}, R\right) d^{3} \mathbf{x}^{\prime}
$$

The smoothed overdensity field is a Gaussian random filed with a variance of

$$
S(R) \equiv \sigma^{2}(R)=\int \frac{k^{2}}{2 \pi^{2}} P(K) \widetilde{W}(k, R)^{2} d k,
$$

where $P(k) \equiv\left\langle|\delta(k)|^{2}\right\rangle$ is the matter power spectrum and $\widetilde{W}(k, R)$ is Fourier transform of the window function. A commonly used window function is the top-hat filter in the real space. Its Fourier transform is

$$
\widetilde{W}(k, R)=\frac{3}{(k R)^{3}}[\sin (k R)-k R \cos (k R)] .
$$

The mass corresponding to the filter scale $R$ is defined as (see also Eq. (2.52) for a comparison)

$$
M(R)=\frac{4}{3} \pi R^{3} \rho_{m}
$$


where $\rho_{m}$ is the background matter density. Then the fraction of mass contained in halos with mass larger than $M(R)$ at $t_{c}$ can be computed by

$$
\begin{aligned}
F(M>M(R))=P\left(\delta>\delta_{c}\left(t_{c}\right)\right) & =\int_{\delta_{c}\left(t_{c}\right)}^{+\infty} \frac{1}{\sqrt{2 \pi S(M)}} \exp \left[-\frac{\delta^{2}}{2 S(M)}\right] d \delta \\
& =\frac{1}{2} \operatorname{erfc}\left[\frac{\delta_{c}\left(t_{c}\right)}{\sqrt{2 S(M)}}\right] .
\end{aligned}
$$

Here we have used Eq. (2.67) to write $S$ as a function of $M$. For CDM, $S$ becomes infinity when M approaches to 0 . Thus from Eq. (2.68), we will conclude that at most half of the total mass is locked in halos. This can be understood as the underdense regions will not end up in halos. But in fact the underdense regions may be enclosed within a larger overdense region so that they will collapse together. Considering this Press and Schechter add a fudge factor of 2 to Eq. (2.68). By changing the filter scale, we can obtain the fraction of mass that is contained in halos with mass between $M$ and $M+d M$. The number density of halos per logarithmic bin of mass, i.e. the halo mass function, is given by

$$
\frac{d n\left(M, t_{c}\right)}{d \ln M}=-2 \frac{\rho_{m}}{M} \frac{\partial F}{\partial M}=-\frac{1}{\sqrt{2 \pi}} \frac{\rho_{m}}{M} \frac{\delta_{c}\left(t_{c}\right)}{\sqrt{S(M)}} \exp \left[-\frac{\delta_{c}\left(t_{c}\right)^{2}}{2 S(M)}\right] \frac{d \ln S(M)}{d \ln M} .
$$

Note that here we have added the fudge factor 2 into the formula. Despite its relative simplicity, the halo mass function computed from Eq. 2.69) has been found to be in reasonable agreement with $N$-body cosmological simulations of CDM (see [93] for a review).

\subsubsection{Extended Press-Schechter Formalism}

As is been shown previously, with the Press-Schechter formalism we can compute the halo mass function from the linear matter power spectrum. But a fudge factor of 2 needs to added by hand in the formalism based on the argument that it does not account for the underdense regions that may be enclosed within a larger overdense region, i.e. the cloud-in-cloud problem [94, 95, 96, 97, 98, 99]. A consistent derivation of the halo mass function goes back to [99], who found that by solving the so-called excursion set problem the fudge factor 2 is automatically accounted for in the solution. This approach is also known as the extended Press-Schechter (EPS) formalism.

As in Section 2.1.5, we smooth the overdensity field with a filter. However, here we will use a different window function which is easier for later calculations, i.e. the sharp-k filter (a top-hat function in Fourier space):

$$
\widetilde{W}\left(k, k_{s}(R)\right)=\Theta\left(k_{s}(R)-k\right) .
$$


Here $\Theta$ is the Heaviside step function. Unlike the top-hat filter in real space, it is a bit ambiguous to define a mass scale corresponding to $k_{s}$. One "natural" treatment is to compute the integration of $W\left(r, k_{s}(R)\right)^{2}$ in real space and identify it with the volume enclosed within the smoothing scale $R$ [100]:

$$
\int_{0}^{\infty} 4 \pi r^{2} W\left(r, k_{s}(R)\right)^{2} d r=\frac{4}{3} \pi R^{3} .
$$

Here $W\left(r, k_{s}(R)\right)$ is the inverse Fourier transform of $\widetilde{W}\left(k, k_{s}(R)\right)$. The relation between $k_{s}$ and $R$ is then given by

$$
k_{s}=\frac{c}{R},
$$

where $c=(9 \pi / 2)^{1 / 3}$. However, as pointed out by [100, 91], this treatment still lacks strong physical motivations. In practice, $c$ can be chosen as a free parameter that needs to be fitted to simulations. For example, [91] find $c=2.5$ (see also [101, 102, 68]) by comparing the semi-analytic halo mass function for warm dark matter with the results from N-body simulations.

The variance of the smoothed field is

$$
S(R)=\int_{0}^{+\infty} \frac{k^{2}}{2 \pi} P(k) \widetilde{W}\left(k, k_{s}(R)\right)^{2} d k=\int_{0}^{k_{s}} \frac{k^{2}}{2 \pi} P(k) d k,
$$

where $P(k)$ is the matter power spectrum. When the filter scale $R \rightarrow+\infty$, we have $S(R) \rightarrow 0$ and $\delta_{R} \rightarrow 0$. As we decrease the filter scale $R, S(R$ increases and perturbations on smaller and smaller scales are included. The change of the smoothed overdensity filed $\Delta \delta_{R}$ is also a Gaussian random field with a mean value of 0 and a variance of

$$
\begin{aligned}
\left\langle\Delta \delta_{R}^{2}\right\rangle & =\int_{0}^{+\infty} \frac{k^{2}}{2 \pi} P(k)\left[\widetilde{W}\left(k, k_{s}(R)+\Delta k_{s}\right)^{2}-\widetilde{W}\left(k, k_{s}(R)\right)^{2}\right] d k \\
& =\int_{k_{s}}^{k_{s}+\Delta k_{s}} \frac{k^{2}}{2 \pi} P(k) d k \\
& =S(R+\Delta R)-S(R) .
\end{aligned}
$$

One of the advantages of using sharp-k filter is that the distribution of $\Delta \delta_{R}$ is independent of the previous value of $\delta_{R}$. Thus if we treat $S(R)$ as the time variable, the trajectory of $\delta_{R}$ can be analogous to a random walk process, or more precisely a Wiener process.

Fig. 2.4 shows one realization of the trajectory of $\delta_{R}$. As can be seen, $\delta_{R}$ starts at $S=0$ and $\delta_{R}=0$. As $S$ increases, $\delta_{R}$ changes in a random way. At $S=S_{1}, \delta_{R}$ is as large as the critical overdensity for collapse $\delta_{c}$. In the language of the PressSchechter theory, the mass element is considered to be contained in a collapsed halo 


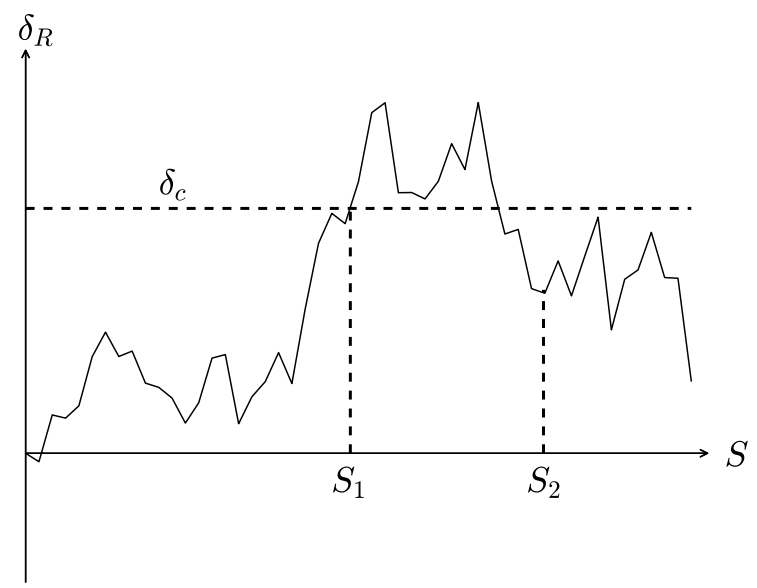

Figure 2.4: A realization of the trajectory of $\delta_{R}$.

with mass $M \geq M_{1}$. Here $M_{1}$ is related to the filter scale by Eq. (2.67). Note that, the change of $\delta_{R}$ is not necessarily positive when $S$ increases. So at $S_{2}>S_{1}, \delta_{R}$ may become smaller than $\delta_{c}$. Then we would conclude that the mass element should not be contained in a collapsed halo with $M \geq M_{2}$, which is in contradiction to the previous conclusion that this element is contained in a halo with $M \geq M_{1}>M_{2}$. Thus the Press-Schechter theory is not self-consistent.

To resolve the inconsistency, the mass element is assigned to a halo when $\delta_{R}$ first exceeding the critical overdensity in EPS formalism. In the following analysis, we will call the probability that $\delta_{R}$ first upcrossing the critical overdensity the first-crossing probability and denote is as $f(S)$. Then with the relation between the halo mass $M$ and $S(R), f(S)$ can be converted to the halo mass function:

$$
\frac{d n(M)}{d \ln M}=-\frac{\rho_{m}}{M} f(S) S \frac{d \ln S}{d \ln M} .
$$

Since the change of $\delta S_{R}$ is independent of the starting value of $\delta_{R}$, the conditional probability that the trajectory first upcrosses $\delta_{c}$ at $S_{1}$ and has a value of $\delta_{R}$ at $S_{2}>S_{1}$ is given by

$$
P_{12}\left(S_{1}, S_{2}\right)=\frac{1}{\sqrt{2 \pi\left(S_{2}-S_{1}\right)}} \exp \left[-\frac{\left(\delta-\delta_{c}\right)^{2}}{2\left(S_{2}-S_{1}\right)}\right] .
$$

Here we have omitted the subscript " $R$ " in $\delta_{R}$. At a specified $S$, the mass element should either contained in a halo with $M \geq M(S)$ or not, so we have

$$
\int_{0}^{S} f\left(S^{\prime}\right) d S^{\prime}+\int_{-\infty}^{\delta_{c}(S)} P(\delta, S) d \delta=1 .
$$

Here the second term on the left-hand side gives the probability that the trajectory has never upcrossed $\delta_{c}$ at smaller $S$. To be more general, a possible dependence in $\delta_{c}$ 
on the halo mass (or equivalently on $S$ ), e.g. in the case of FDM, has been added. From previous discussions, we know that

$$
P(\delta, S)=\frac{1}{\sqrt{2 \pi S}} \exp \left(-\frac{\delta^{2}}{2 S}\right)-\int_{0}^{S} f\left(S^{\prime}\right) P_{12}\left(S^{\prime}, S\right) d S^{\prime} .
$$

Substituting Eq. (2.78) into Eq. (2.77), and integrating over $\delta$, we obtain

$$
\int_{0}^{S} f\left(S^{\prime}\right) \operatorname{erfc}\left[\frac{\delta_{c}(S)-\delta_{c}\left(S^{\prime}\right)}{\sqrt{2\left(S-S^{\prime}\right)}}\right] d S^{\prime}=\operatorname{erfc}\left[\frac{\delta_{c}(S)}{\sqrt{2 S}}\right]
$$

where erfc is the complementary error function.

For CDM, $\delta_{c}$ is independent of $S$, Eq. (2.79) can be solved analytically and the solution is given by

$$
f(S)=\frac{\delta_{c}}{\sqrt{2 \pi S}} \exp \left[-\frac{\delta_{c}^{2}}{2 S}\right] \frac{1}{S} .
$$

Substituting Eq. 2.80) into Eq. (2.75), the exactly same formula for the halo mass function as Eq. 2.69) is obtained. But here we do not need to add any fudge factor, i.e. the cloud-in-cloud problem is solved.

Another important application of the EPS formalism is to calculate the probability of finding the progenitors of a halo at an earlier time. Consider that a smoothed overdensity $\delta_{R}$ first upcrossed the critical value $\delta_{c}\left(t_{0}\right)$ at $S=S_{0}$ and $t=t_{0}$. Within the EPS formalism, it is considered to be contained in a halo with mass $M_{0}$. Now we can ask what is the probability that we find this mass element contained in a halo with mass $M_{1}<M_{0}$ at an earlier time $t_{1}=t_{0}-\Delta t$. As discussed before, the critical overdensity for collapse is larger at earlier time. So the problem to be solved is similar to the excursion set problem solved above except that the trajectory of $\delta_{R}$ now originates from $S=S_{0}$ and $\delta_{R}=\delta_{c}\left(t_{0}\right)$. Define new variables $\bar{S}=S-S_{0}$ and $\bar{\delta}_{R}=\delta_{R}-\delta_{c}\left(t_{0}\right)$. The probability finding the mass element in $M_{1}$ at $t_{1}$, i.e. $f_{0 \rightarrow 1}$, is equal to the probability $\bar{\delta}_{R}$ first upcrossing $\bar{\delta}_{c}=\delta_{c}\left(t_{1}\right)-\delta_{c}\left(t_{0}\right)$ at $\bar{S}=S_{1}-S_{0}$. The function $f_{0 \rightarrow 1}$ satisfies the same integral equation as Eq. 2.79). We just need to replace $\delta$ with $\delta-\delta_{c}\left(t_{0}\right), S$ with $S-S_{0}$ and $\delta_{c}$ with $\delta_{c}\left(t_{1}\right)-\delta_{c}\left(t_{0}\right)$. The mean number of the halo's progenitors with a mass between $M_{1}$ and $M_{1}+d M_{1}$ at an earlier time $t_{1}$ can then be computed as

$$
d N=f_{0 \rightarrow 1}\left(S_{0}, S_{1}\right) \frac{M_{0}}{M_{1}} \frac{d S}{d M_{1}} d M_{1} .
$$

Specially, for CDM the solution of $f_{0 \rightarrow 1}$ takes the form of

$$
f_{0 \rightarrow 1}\left(S_{0}, S_{1}\right)=\frac{\delta_{c}\left(t_{1}\right)-\delta_{c}\left(t_{0}\right)}{\sqrt{2 \pi\left(S_{1}-S_{0}\right)}} \exp \left[-\frac{\left(\delta_{c}\left(t_{1}\right)-\delta_{c}\left(t_{0}\right)\right)^{2}}{2\left(S_{1}-S_{0}\right)}\right] \frac{1}{S_{1}-S_{0}} .
$$




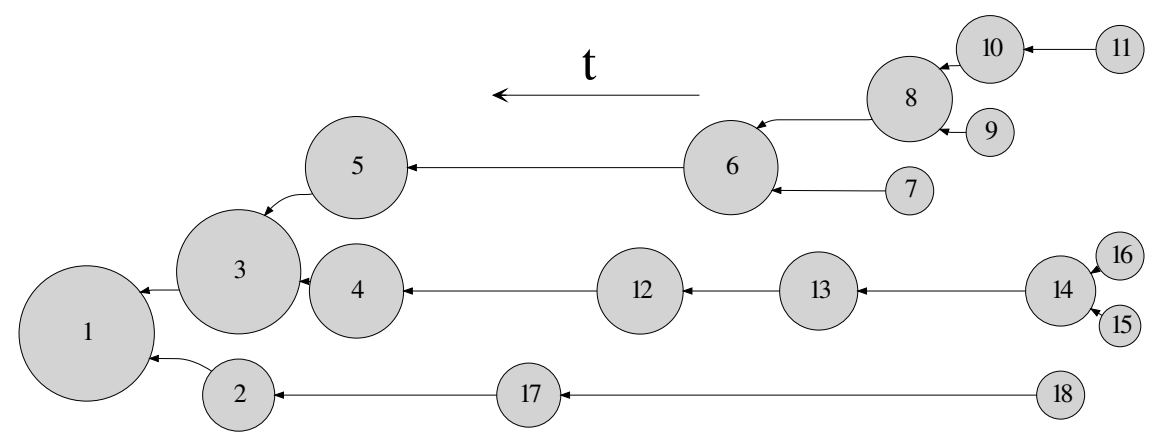

Figure 2.5: A schematic merger tree.

\subsubsection{Merger Tree}

In models of hierarchical structure formation, the cosmic structure forms bottomup, i.e. small halos form first, and then merge into larger and larger halos. The merger history is usually described by the merger tree, which links halos with their progenitors. Fig. 2.5 shows a schematic merger tree. The radius of the circle represents the mass of a halo. For simplicity, we consider only binary mergers. It gives a good description of the merger history as long as we look on sufficient small time scales so that within one time step only binary merger happens.

Merger trees can either be extracted from cosmological simulations, e.g. N-body simulations, or be constructed stochastically based on the mean progenitor distribution discussed in the previous subsection. Merger trees obtained in the first way have the advantage that cosmological simulations more reliably capture the physics of structure formation without simplifications used to derive the EPS formalism. But the cost is that merger trees are limited by the dynamical range resolved by the simulations. Additionally, there are uncertainties in identifying halos and their progenitors. Besides, cosmological simulations usually need very high computational costs. On the contrary, constructing merger trees using Monte-Carlo algorithms is much less expensive so that it is possible to generate a large sample of trees with high mass resolution. It has been shown that Monte-Carlo merger trees can well reproduce the statistics of cosmic structure as seen in direct simulations (see e.g. [103, 104, 105]). Furthermore, as shown in [106], Monte-Carlo algorithms can be used to augment the resolution of merger trees from cosmological simulations. The statistics of augmented merger trees are found to be well consistent with direct higher resolution simulations. In the following discussions, I will focus on constructing Monte-Carlo merger trees.

The EPS formalism gives the probability of finding one halo's progenitor with a specific mass (see Eq. (2.81)), but it does not tell us how many progenitors the halo 
(descendant halo) has and the conditional probability of one progenitor on the others. For instance, if we draw one progenitor $M_{1}$ for the halo $M_{0}$ from the distribution Eq. 2.81), the remained mass $M_{0}-M_{1}$ in the descendant halo will limit the mass that the second progenitor can have. The EPS formalism provides no information about the conditional probability of the second progenitor on the first one, thus additional assumptions are needed [88]. Based on different assumptions, different kinds of algorithms have been developed.

The simplest way is to consider only binary merger, e.g. the algorithm proposed in [100] and the improved version in [105] (hereafter Cole2000). In such kind of algorithm, the second progenitor is assigned a mass of $M_{2}=M_{0}-M_{1}$. To account for the progenitors below the mass resolution, a small fraction of mass is assumed to be smoothly accreted so that $M_{2}=M_{0}-M_{1}-M_{\text {acc }}$. Such algorithms are easy to implement but have the limitations that one halo can have at most two progenitors and the progenitor distribution is by-hand symmetrized, i.e. the mean number of progenitors with mass $M_{1}$ is the same as that of progenitors with mass $M_{0}-M_{1}$ (or $\left.M_{0}-M_{1}-M_{\text {acc }}\right)$.

Without the assumption of binary mergers, [107] proposed anther way to build merger trees. They keep drawing progenitors from the distribution Eq. 2.81. A new progenitor is rejected if the total mass of the progenitors exceeds $M_{0}$. When the mass remained is less than the mass resolution, the process above is stopped and the remained mass is assumed to be smoothly accreted. However, due to the rejection procedure, the progenitors drawn later will not have the same probability distribution as previous ones. Thus the mean distribution of progenitors is not exactly the same as Eq. 2.81). Better algorithms can be achieved if the smooth accretion by each progenitor during one time step is taken into account [107].

In the above algorithms, progenitors are drawn for one halo based on the EPS formalism and some extra assumptions. But since the EPS formalism only gives the average properties of the progenitors, the extra assumptions need to be chosen carefully so that the merger trees constructed are consistent with the EPS formalism on average. A different approach is proposed by [108]. Instead of drawing progenitors one by one for one descendant halo, it generates a bunch of progenitors for hundreds of descendant halos based on the mean progenitor distribution and the mean number of progenitors. Then the progenitors are distributed among the descendant halos assuming mass conservation. Finally, a single realization is randomly drawn from the hundreds of realizations. As shown in [109], compared to the algorithms discussed 
previously this algorithm is the only one that is fully consistent with the EPS formalism. However, in each time step a large table of progenitor distribution needs to be stored.

More algorithms have been developed to solve different problems in the algorithms mentioned above (see e.g. [110, 109, 111]). I will follow the Cole2000 algorithm since it is relatively simple compared to the others and as mentioned before, if we chose a sufficiently small time step size considering only binary mergers can be a good approximation.

Given a halo with mass $M_{0}$ at $t_{0}$, the mean distribution of its progenitors is given by Eq. (2.81). Assume that the minimum mass we would like to resolve in the merger tree, i.e. the mass resolution, is $M_{\text {res }}$. Then the mean number of progenitors with mass $M_{\text {res }}<M_{1}<M_{0} / 2$ is given by

$$
N_{p}=\int_{M_{\mathrm{res}}}^{M_{0} / 2} f_{0 \rightarrow 1}\left(S_{0}, S_{1}\right) \frac{M_{0}}{M_{1}} \frac{d S}{d M_{1}} d M_{1} .
$$

Besides, there is some fraction of mass in $M_{0}$ that is from progenitors below the mass resolution. The fraction can be computed as

$$
F=\int_{0}^{M_{\mathrm{res}}} f_{0 \rightarrow 1}\left(S_{0}, S_{1}\right) \frac{d S}{d M_{1}} d M_{1} .
$$

In the Cole2000 algorithm, a small time step size $\Delta t$ is chosen so that at the earlier time $t_{0}-\Delta t, N_{p}$ is much smaller than 1 . This ensures that during each time step only binary mergers are likely to happen. Then a random number $N_{d}$ is drawn from the uniform distribution over $(0,1)$. If $N_{d}>N_{p}$, no merger above the mass resolution is assumed to happen. The halo mass is reduced to $(1-F) M_{0}$, which accounts for the smooth accretion of halos with mass below $M_{\text {rmres }}$. If $N_{d} \leq N_{p}$, a progenitor with mass $M_{\text {res }}<M_{1}<M_{0} / 2$ is drawn randomly following the distribution function Eq. (2.81). The second progenitor is assigned a mass of $M_{2}=(1-F) M_{0}-M_{1}$. Here the smooth accretion has also been accounted for. Such procedure is repeated for each progenitor backward at successive time steps until the progenitor mass reaches $M_{\text {res }}$ (see Fig. 2.5).

Note that as mentioned before, here we have explicitly assumed that the probability finding a progenitor $M_{1}$ is the same as a progenitor $M_{2}=(1-F) M_{0}-M_{1}$. In general, this symmetry is not present in the EPS formalism Eq. 2.81). However, [105] found that by restricting the first progenitor's mass to be smaller than $M_{0} / 2$ and accounting for the smooth accretion below the mass resolution, the merger trees generated can reproduce progenitor distributions that agrees reasonably well with the EPS formalism on average. 


\subsection{Semi-analytic Model of Galaxy Formation}

In the previous section, I have introduced some of the basic knowledge and methods that are widely used in the study of cosmic structure formation. Most of those discussions have been concentrated on analytic and approximate treatments, i.e. semianalytic models (SAMs). The models are semi-analytic in the sense that they try to describe the physical processes in structure formation with a series of analytic formulas. These analytic formulas may come from analytic solutions to the simplified problems, or from some empirical formulas when the process is too complicated to solve analytically. Usually there is a set of parameters in SAMs that are needed to be calibrated to N-body/hydrodynamical simulations and observations. But compared to direct simulations, SAMs provide us a much less expensive way to explore a lot of different cosmological and astrophysical models.

Applying and extending SAMs to the case of FDM models will be the main task of this dissertation. But before going into the details, it will be helpful to have a general picture of how SAMs work. Although I have only considered dark matter so far, SAMs have also been widely used for investigating galaxy formation which involves complex baryonic physics.

The earlier work on SAMs of galaxy formation dates back to [112], who have used the Press-Schechter theory to describe the hierarchical structure of dark matter halos and combined it with a model of gas cooling to study galaxy formation. This ideal was further developed into a self-consistent model by [113, 114, 115]. In the past three decades, a lot of improvements have been made to SAMs (see e.g. [116, 117, 118] for reviews). Based on different treatments of the baryonic processes such as gas cooling, star burst, feedback from supernovae and Active Galactic Nucleus (AGN), and different ways of calibrations, a lot of models have been developed. Several popular models are from Cole et al. [105], Croton et al. [119, 120] and Somerville et al. [121.

Fig. 2.6 shows the main recipes of SAMs. First, a cosmological model is chosen. Then merger trees are constructed either from N-body simulations or from the EPS formalism. Baryons are assumed to trace the distribution of dark matter. Thus each dark matter halo is adjoined to several baryonic components, e.g. hot gas, cold gas, and stars. Each component, including the dark matter, is evolved according to a submodel. When a dark matter halo encounters another halo in the merger tree, the smaller one will be treated as a subhalo (substructure) and the galaxy associated with it becomes a satellite galaxy. The subhalo and satellite galaxy are then evolved in 


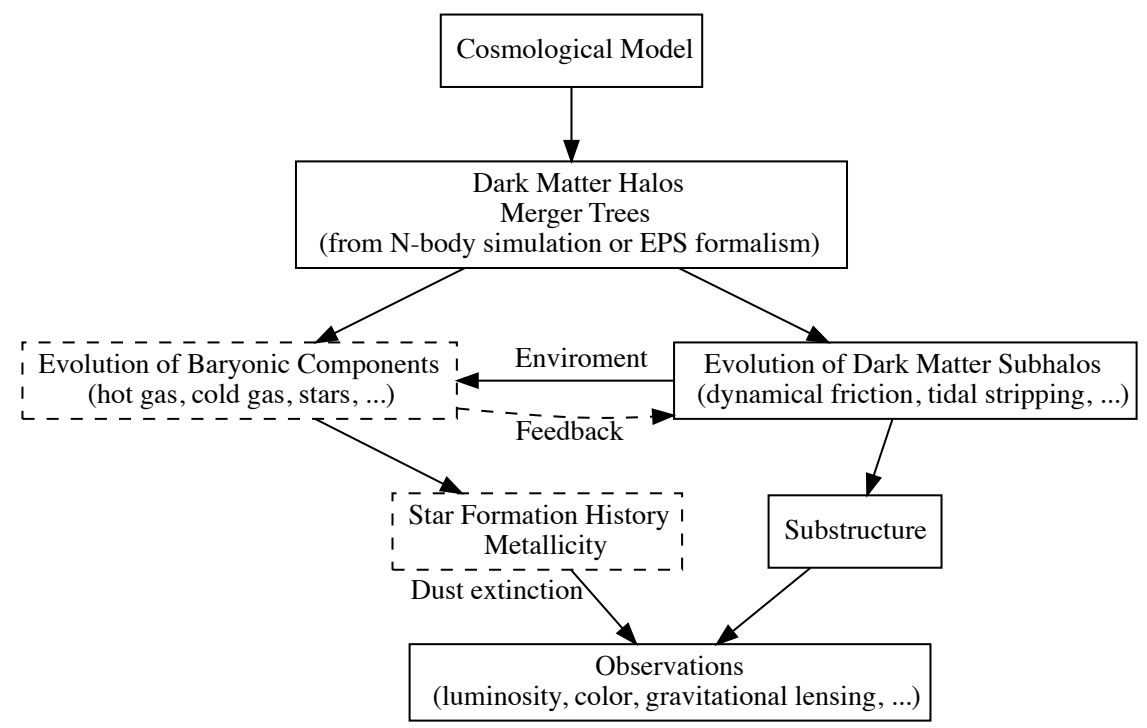

Figure 2.6: A schematic diagram of semi-analytic model of galaxy formation.

the gravitational potential of the host halo until it is considered to be fully merged with the host. The (sub)halos provide the environment for galaxy formation. On the other hand, the feedback in the baryonic components can also affect the distribution of dark matter. Combining all these submodels, one solves a large number of coupled differential equations, representing a lot of submodels for different components. The star formation rate and metallicity can then be obtained and converted using some models for dust extinction into observables such as the luminosity and color. Besides, the population and spatial distribution of the subhalos can be directly compared to observations from gravitational lensing.

In this dissertation, I will concentrate on the evolution of dark matter substructure and possible comparison with observations (marked by solid boxes in Fig. 2.6). The physical processes related to baryons (dashed boxes in Fig. 2.6) will be the next stage of this work.

\subsection{Implementation of FDM in SAMs}

With the knowledge of the linear perturbation theory and the spherical collapse model as described in Section 2.1, it is possible to build a SAM for FDM just as for CDM. In this dissertation, I make use of the publicly available semi-analytic code GALACTICUS 122 and modify it to include several aspects of FDM physics. GALACTICUS is a 
highly modular semi-analytic code for galaxy formation, thus it is capable to be extended to include alternative models. For instance, [91] apply it to the case of warm dark matter (WDM) and investigate the halo mass function and progenitor mass function in this model. Excellent agreement with N-body simulations of WDM has been found. [123] further extend it to include nonlinear evolution of subhalos in the host halo for both CDM and WDM. Our modifications for FDM follow a similar approach, but also include some unique features in FDM models.

\subsubsection{A New Determination of the HMF for FDM}

The most direct way to obtain the HMF is to perform cosmological simulations. In the nonrelativistic limit, the evolution of FDM can be described by a complex wave function $\psi$ which satisfies the Schrödinger-Poisson (SP) equations [51, 25]. Thus one could investigate the nonlinear structure formation in FDM models by solving the SP equations with cosmological initial conditions [27, 29, 4]. However, due to the rapid oscillations of the wave function, extremely high resolution both in space and time is necessary to correctly resolve high-velocity flows [29, 33]. Even the state-of-theart simulations in 29] can only fully resolve a box of $2 \mathrm{Mpc}$ on each side, which is too small for determining the HMF. One alternative way is to do standard N-body simulations but with FDM initial conditions [33, 34, 65]. With such simulations, fitting formulas for the FDM HMF has been obtained in [33, 65]. It is argued that the quantum pressure arising in FDM models does not have a significant impact on the nonlinear formation of moderate and large halos at lower redshifts 6 . However, to resolve accurately the abundance of the smallest halos, simulations solving the full dynamics as in [29, 62, 124] are still essential. Other approaches accounting for the quantum pressure in N-body or hydrodynamic simulations have also been making progress (see e.g. [125, 126, 63, 127, 92]).

On the other hand, we can make use of the EPS formalism as discussed in Section 2.1.6 to compute the HMF for FDM. Compared to CDM, there are two important things we needs to account for:

(1) a suppression in the linear power spectrum (transfer function) on scales smaller than the quantum Jeans length;

(2) the growth of FDM overdensity is not scale-independent, thus leads to a scaledependent critical overdensity for collapse.

\footnotetext{
${ }^{6}$ The quantum pressure still plays an important role in suppressing the linear power spectrum which is used to generate the initial conditions.
} 
Then the HMF for FDM can be obtained by solving the excursion set problem (see Section 2.1.6):

$$
\frac{d n(M)}{d \ln M}=-\frac{\rho_{m}}{M} f(S) S \frac{d \ln S}{d \ln M},
$$

where $f(S)$ is the first-crossing distribution and satisfies

$$
\int_{0}^{S} f\left(S^{\prime}\right) \operatorname{erfc}\left[\frac{\delta_{c}(S)-\delta_{c}\left(S^{\prime}\right)}{\sqrt{2\left(S-S^{\prime}\right)}}\right] d S^{\prime}=\operatorname{erfc}\left[\frac{\delta_{c}(S)}{\sqrt{2 S}}\right]
$$

The FDM density power spectrum can be written as

$$
P_{\mathrm{FDM}}=T_{\mathrm{FDM}}^{2} P_{\mathrm{CDM}},
$$

where $P_{\mathrm{CDM}}$ denotes the power spectrum in the CDM model, and $T_{\mathrm{FDM}}$ characterizes the suppression of FDM power spectrum relative to CDM. A fitting formula for $T_{\mathrm{FDM}}$ is found in [25]:

$$
T_{\mathrm{FDM}}=\frac{\cos x^{3}}{1+x^{8}}
$$

where

$$
x=1.61 m_{22}^{1 / 18} \frac{k}{k_{J, \mathrm{eq}}} .
$$

Here $m_{22} \equiv m_{a} /\left(10^{-22} \mathrm{eV}\right)$ and $k_{J \text {,eq }} \approx 9 m_{22}^{1 / 2} \mathrm{Mpc}^{-1}$ is the quantum Jeans wave number at matter-radiation equality which can be computed from Eq. 2.40). More accurate power spectra for FDM or a mixture of FDM and CDM can be obtained using AxionCAMB 7 [55, 66] which includes FDM as one component in the publicly available Boltzmann code CAMB [128].

Fig. 2.7 shows $T_{\mathrm{FDM}}(k)$ for different FDM masses computed using AxionCAMB. As can be seen, $T(k)$ drops steeply at large $k$. A sharp cutoff is shown at roughly the Jeans wave number at matter-radiation equality (see the vertical dashed lines). It means the small-scale perturbations, as the seeds for nonlinear structure formation, will be largely suppressed below the Jeans scale. As the FDM mass increases, the suppression happens at smaller and smaller scales, thus FDM will behave more like CDM. For comparison, we also consider one model in which FDM and CDM each make up half of the total dark matter (thick dashed curve). We can see that the suppression of matter power spectrum happens roughly at the same scales as in the pure FDM case with the same FDM mass, but the suppression is weaker.

For CDM, in the matter-dominated era different modes of linear overdensity all grow as $a$, independent of the corresponding wave number $k$ (see Section 2.1.2).

\footnotetext{
${ }^{7}$ https://github.com/dgrin1/axionCAMB
} 


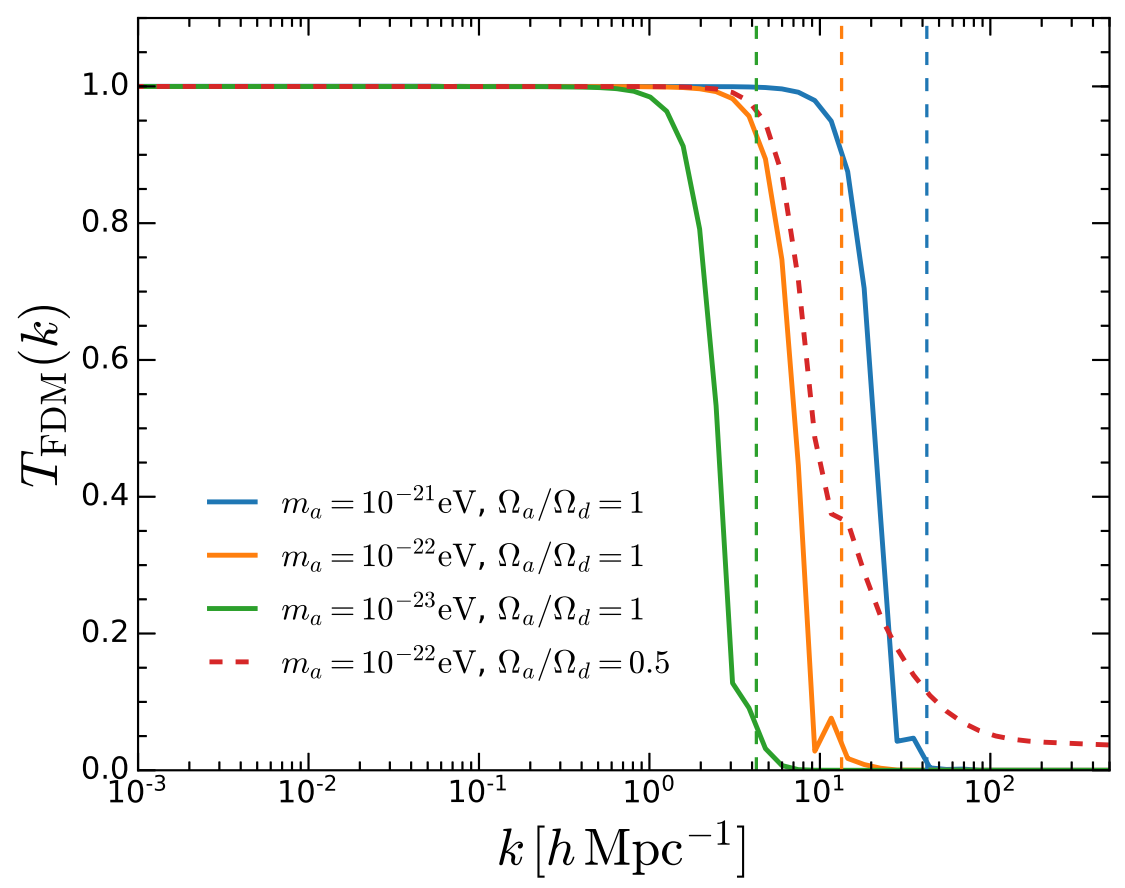

Figure 2.7: Transfer function $T_{\mathrm{FDM}}(k)$, as defined in Eq. 2.88, for FDM with different masses and fractions. The results is derived using the publicly available code AxionCAMB. Here we have also considered a model in which FDM only makes up half of the dark matter, i.e. $\Omega_{a} / \Omega_{d}=0.5$ (thick dashed curve). The vertical dashed lines mark the Jeans wave numbers at matter-radiation equality for different FDM masses. 
Furthermore, if we assume spherical collapse as discussed in Section 2.1.4, overdense regions will collapse to halos once the overdensities reach the critical value $\delta_{c} \approx 1.686$, which does not depend on the size of the overdense region.

However, for FDM as shown in Section 2.1.4, modes with small wave numbers collapse later compared to CDM. Thus a higher critical overdensity is required for an overdense region to collapse at the same redshift as CDM. As mentioned in Section 2.1.4, detailed simulations of spherical collapse are needed to find the dependence of the critical overdensity on $k$. As an alternative way, [55] proposed that we can estimate the critical overdensity for FDM from the scale-dependent growth of linear overdensities, i.e. $D(k, z)$. The basic idea is as follows. Compared to CDM, the growth of FDM linear overdensity is suppressed by a factor of

$$
\mathcal{G}(k, z)=\frac{D_{\mathrm{CDM}}(z)}{D_{\mathrm{FDM}}(k, z)}
$$

Here we have assumed a matter-dominated universe so that $D_{\mathrm{CDM}}$ only depends on $z$. Now suppose that we have an initial overdensity $\delta_{i}^{c}$ at $z_{i}$. In the CDM case, this overdensity reaches the critical value $\delta_{c}$ at $z_{c}$. With the same initial value, in the FDM case, at $z_{c}$ the overdensity will be $\delta_{\mathrm{FDM}}\left(z_{c}\right)=\delta_{c} / \mathcal{G}(k, z)<\delta_{c}$. To reach the critical value $\delta_{c}$ at $z_{c}$, the initial overdensity in the FDM case needs to be $\delta_{i, F D M}^{c}=\mathcal{G}(k, z) \delta_{i}^{c}$. In other words, to collapse at the same redshift, the critical overdensity in FDM model needs to be larger than $\mathrm{CDM}$ by a factor of $\mathcal{G}(k, z)$ :

$$
\delta_{c}^{\mathrm{FDM}}(k, z)=\mathcal{G}(k, z) \delta_{c}^{\mathrm{CDM}}(z) \approx \mathcal{G}(k, z) \frac{1.686}{D_{\mathrm{CDM}}(z)} .
$$

Here we have used the formula of critical overdensity for CDM, Eq. 2.63.

The relative amount of growth between $\mathrm{CDM}$ and FDM, $\mathcal{G}$, can be obtained by solving the evolution of linear density perturbations:

$$
\mathcal{G}(k, z)=\frac{\delta_{\mathrm{CDM}}(k, z) \delta_{\mathrm{CDM}}\left(k_{0}, z_{h}\right)}{\delta_{\mathrm{CDM}}\left(k, z_{h}\right) \delta_{\mathrm{CDM}}\left(k_{0}, z\right)} \frac{\delta_{\mathrm{FDM}}\left(k, z_{h}\right) \delta_{\mathrm{FDM}}\left(k_{0}, z\right)}{\delta_{\mathrm{FDM}}(k, z) \delta_{\mathrm{FDM}}\left(k_{0}, z_{h}\right)},
$$

where $k_{0}=0.002 \mathrm{hMpc}^{-1}$ is a pivot scale, and $z_{h}$ is chosen to be large enough so that at the relevant redshift the shape of CDM power spectrum has frozen in (in the matter-dominated era). We set $z_{h}=300$ as in [55].

Ignoring the possible time dependence in $\mathcal{G}$, a fitting function for $\mathcal{G}$ is given in [2] based on numerical results from AxionCAMB:

$$
\mathcal{G}(M)=h_{\mathrm{F}}(x) \exp \left(a_{3} x^{-a_{4}}\right)+\left[1-h_{\mathrm{F}}(x)\right] \exp \left(a_{5} x^{-a_{6}}\right)
$$


where

$$
\begin{aligned}
x & =M / M_{\mathrm{J}}, \\
h_{\mathrm{F}}(x) & =\frac{1}{2}\left\{1-\tanh \left[M_{\mathrm{J}}\left(x-a_{2}\right)\right]\right\} \\
M_{\mathrm{J}} & =10^{8} a_{1}\left(\frac{m_{\mathrm{a}}}{10^{-22} \mathrm{eV}}\right)^{-3 / 2}\left(\frac{\Omega_{\mathrm{m}} h^{2}}{0.14}\right)^{1 / 4} h^{-1} M_{\odot} .
\end{aligned}
$$

Here the $k$ dependence has been converted to the dependence on the halo mass via $M \equiv \frac{4}{3} \pi(\pi / k)^{3} 8$. The best-fitting parameters are found to be $\left\{a_{1}, a_{2}, a_{3}, a_{4}, a_{5}, a_{6}\right\}=$ $\{3.4,1.0,1.8,0.5,1.7,0.9\}$. Note that ignoring the time dependence has little effect on the HMF for FDM masses $\left(m_{a} \gtrsim 10^{-24} \mathrm{eV}\right)$ and redshifts $(z \lesssim 15)$ that are concerned in this dissertation, because the critical overdensity increases rapidly at small scales and leads to a sharp cutoff of the HMF before the time dependence of $\mathcal{G}$ becomes important (see also [2]).

Figs 2.8 and 2.9 show the critical overdensity for collapse $\delta_{c}$ at $z=0$ for different FDM fractions and different FDM masses, respectively. On large scales (large $M$ ), $\delta_{c}$ stays constant just as CDM. But on small scales (small $M$ ), $\delta_{c}$ for FDM becomes larger than that for CDM, indicating that smaller FDM halos are more difficult to form. For the same FDM mass, the critical overdensity for collapse start to increase at almost the same scale but the increase is larger for larger fraction of FDM. For pure FDM models, the scale at which the $\delta_{c}$ start to deviate from CDM increases with decreasing FDM mass.

Knowing the matter power spectrum and critical overdensity for collapse, the only task remaining is to solve the excursion set problems Eqs. 2.85) and 2.86). But before doing that, let us look at a more accurate model for the HMF that affects both CDM and FDM. In the original Press-Schechter (PS) formalism, the collapse of halos is assumed to be spherically symmetric. But in the real case, the overdense region may be not ideally spherical. It has been found that the original PS formalism does not agree perfectly with $N$-body simulations of CDM. It tends to underestimate the abundance of massive halos and overestimate the abundance of low-mass ones. A improved formula is proposed by Sheth and Tormen (hereafter "ST") [129], which fits accurately to $N$-body simulations of CDM:

$$
f(S)=A \sqrt{\frac{1}{2 \pi}} \sqrt{q} \nu\left[1+(\sqrt{q} \nu)^{-2 p}\right] \exp \left(-\frac{q \nu^{2}}{2}\right) \frac{1}{S}
$$

\footnotetext{
${ }^{8}$ Here we have assumed a top-hat filter when doing the conversion. Another choice is using the sharp-filter so that $M \equiv \frac{4}{3} \pi(a / k)^{3}$ with $a \sim 2.5[91$. But we have not explored the latter choice so far and will leave it to future work.
} 


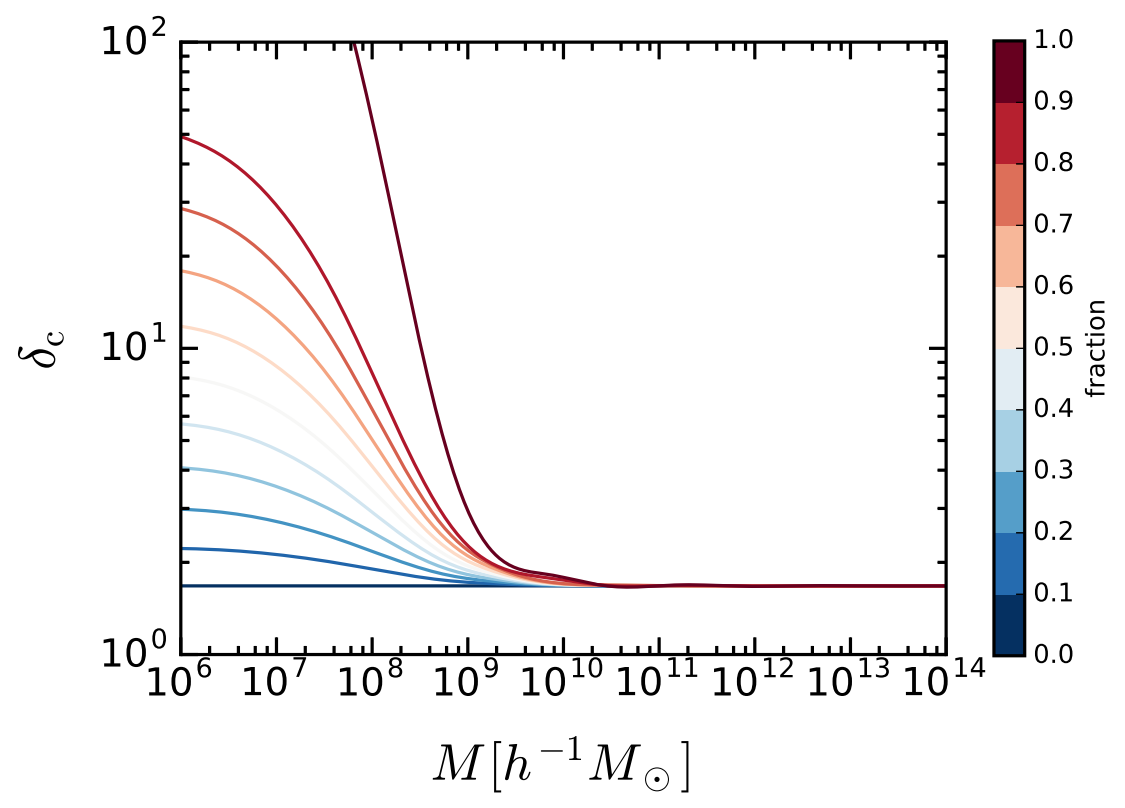

Figure 2.8: Critical overdensity for collapse at $z=0$ with different FDM fractions $\Omega_{a} / \Omega_{d}$. The fractions range from 0 to 1 with a step size of 0.1 . The FDM mass is set to $10^{-22} \mathrm{eV}$. Reproduced from [1], Fig. 1 .

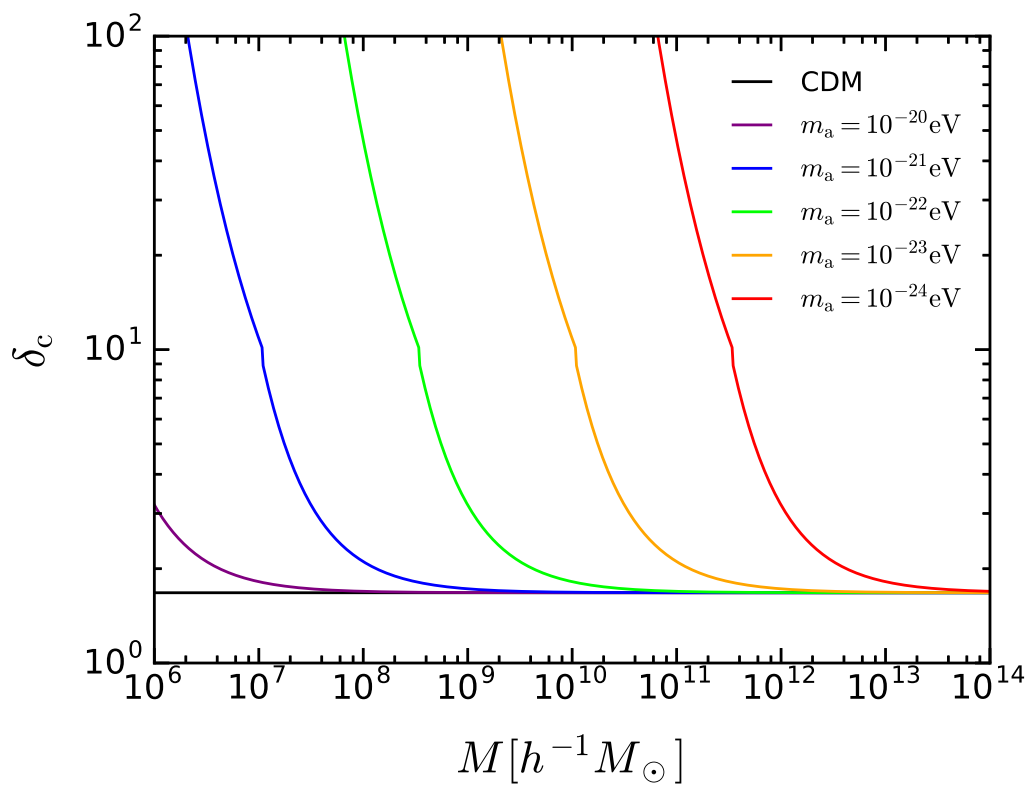

Figure 2.9: Critical overdensity for collapse at $z=0$ with different FDM masses (based on the fitting formula from [2]) compared to standard CDM. Reproduced from [1], Fig. 2. 
where $\nu \equiv \frac{\delta_{c}(S)}{\sqrt{S}}, A=0.3222, p=0.3$, and $q=0.707$. Later, [130] show that Eq. 2.97 can be derived by considering ellipsoidal collapse. According to the Zel'dovich approximation (see [131] for a review), an ellipsoid overdensity collapses most rapidly along the shortest axis. If we define the collapse of a halo as the time when the longest axis collapses, the time of collapse in general will be later than that in the spherical collapse model. The delay of collapse is more significant for less massive halos because they are more influenced by the external tidal forces. In the ellipsoidal collapse model, the critical overdensity for collapse is found to be well approximated by 130

$$
\delta_{c}^{\mathrm{ep}}(S)=\delta_{c}^{\mathrm{sp}} \sqrt{q}\left[1+b\left(\frac{1}{q \nu^{2}}\right)^{c}\right]
$$

where $b=0.5$ and $c=0.6$. This formula remaps the critical overdensity in the spherical collapse model $\delta_{c}^{s p}$ to the one in the ellipsoid collapse model $\delta_{c}^{e p}$. With this remapping, 132 solve the excursion problem and find that the obtained HMF is very close to fitting function Eq. (2.97). Note that the remapping, Eq. (2.98), is derived and calibrated for CDM. For FDM halos with mass $M$ much larger than the Jeans mass at matter-radiation equality $M_{\mathrm{J}, \mathrm{eq}}$, this remapping is expected to work also for FDM since on these scales FDM behaves like CDM. But for FDM halos with $M<M_{\mathrm{J}, \mathrm{eq}}$, FDM behaves very differently from CDM due to quantum pressure before the mass scale exceeds the Jeans mass at the corresponding time. Thus this remapping may be inaccurate and needs to be recalibrated to simulations of FDM. As an approximation, in this dissertation we use the remapping for all FDM halos.

One of the important results of the remapping Eq. 2.98) is that $\delta_{c}$ is not constant any more even for CDM. Thus the integral equation Eq. 2.86 can not be solved analytically.

In previous works [55, 32], the HMF for FDM was computed using directly the ST formula, Eq. (2.97), but with a redefined critical overdensity for collapse as defined in Eq. (2.91). However, the ST formula was obtained for CDM whereas for a different critical overdensity the solution for the first crossing distribution $f(S)$ should be different. Strictly speaking, it is not self-consistent to substitute the critical overdensity for FDM directly into the solution of $f(S)$ for CDM. For this reason, we solve $f(S)$ from the integral equation Eq. 2.86) using a similar method as in [91]. However, instead of a trapezoid rule for the integration, we implemented a mid-point rule that is more stable for our case (see Appendix A] [1]. 


\subsubsection{Modification of the Tree Building Algorithm}

In this dissertation, the merger trees are built using the Cole2000 algorithm [105] implemented in GaLACTICUS. The details can be found in Section 2.1.7. First, a set of root halos $\left\{M_{i}\right\}$ are drawn at redshift $z=0$. Given a root halo mass, the algorithm proceeds to construct a merger history by successively drawing branching events assuming binary mergers. The backwards evolution of a branch of the generated tree is considered complete if the relevant halo has a mass below the mass resolution limit which is a free parameter.

The branching probability is estimated from the conditional mass function computed with the EPS formalism at two redshifts very close to each other assuming spherical collapse (see 2.81). Since the collapse of halos is not perfectly spherical as mentioned before, the branching probability calculated in this way does not accurately match the simulations. Therefore, we use the [133] modifications to the branching probability 9 . Given a halo $M_{0}$ at $t_{0}$. The mean number of its progenitors with mass $M_{\text {res }}<M_{1}<M_{0} / 2$ at an earlier time $t_{2}=t_{0}-\Delta t$ is given by

$$
N_{p}^{\text {mod }}=\int_{M_{\min }}^{M / 2} \frac{M_{0}}{M_{1}} f_{0 \rightarrow 1}\left(S_{0}, S_{1}\right) G\left[\delta_{c}, S\left(M_{0}\right), S\left(M_{1}\right)\right] \frac{d S}{d M_{1}} d M_{1} .
$$

Here, $M_{\min }$ is the lowest mass of resolved halos and $G\left[\delta_{\mathrm{c}}, S\left(M_{0}\right), S\left(M_{1}\right)\right]$ is an empirical modification to Eq. 2.83) obtained by [133]:

$$
G\left[\delta_{\mathrm{c}}, S\left(M_{0}\right), S\left(M_{1}\right)\right]=G_{0}\left(\frac{S\left(M_{1}\right)}{S\left(M_{0}\right)}\right)^{\gamma_{1} / 2}\left(\frac{\delta_{c}^{2}}{S\left(M_{0}\right)}\right)^{\gamma_{2} / 2},
$$

with $G_{0}=0.57, \gamma_{1}=0.38$ and $\gamma_{2}=-0.01$. Note that this modification is calibrated to CDM $N$-body simulations, thus a recalibration may be needed for other dark matter models. Nevertheless, we use the same form and parameters and leave the calibration for FDM to future work when sufficiently large FDM simulations are available. As we show later, the HMF for FDM obtained from the merger trees fits the HMF derived above reasonably well even without a recalibration.

Additionally to binary mergers, the algorithm also accounts for smooth accretion. In the case of CDM, this corresponds to merging events in which the main halo accretes a small halo whose mass is below the mass resolution (see Eq. (2.84)). For FDM, however, there is another source of smoothly accreted material, namely matter

\footnotetext{
${ }^{9} \mathrm{~A}$ remapping of the barrier is used when calculating the HMF. But here as in [91, we use the 133. modifications to the branching probability instead of solving the excursion set problem with a remapped critical overdensity.
} 
that is not locked up in a halo. The smooth accretion rate from this effect can be estimated by 91

$$
\left.\frac{d F}{d t}\right|_{\text {smooth }}=\frac{d f_{n}}{d t} G\left(\delta_{c}, S\left(M_{0}\right), S_{\max }\right) .
$$

Here,

$$
f_{n}=1-\int_{0}^{S_{\max }} f(S) d S .
$$

[91] find that if this effect is not included, the mass of halos at higher redshifts will be overestimated for non-CDM models.

In addition to smooth accretion, we note that for FDM the probability to find any progenitors of a small halo with masses close to the HMF low-mass cutoff is very small. Therefore, we add an additional criterion for the termination of a tree branch: if the mass of a halo is small and the probability of finding any of its progenitors is less than $P_{\min }$, the algorithm will terminate the tree branch even if the node's mass is larger than the mass resolution. Physically, such halos correspond to those forming by collapsing density perturbations at that time as opposed to merging of smaller halos or smooth accretion.

Note that the branching criteria $P_{\text {min }}$ must be chosen carefully. Like the HMF, the branching probability $f_{0 \rightarrow 1}$ should drop off at small masses. Owing to numerical artifacts, the derived branching probability becomes inaccurate when the progenitor mass approaches the cutoff, thus $P_{\min }$ must be chosen sufficiently large to avoid these artefacts. On the other hand, if $P_{\min }$ is too large, the number of progenitors will be underestimated. Based on these two considerations, we choose different $P_{\min }$ for different FDM density fractions and masses.

\subsubsection{FDM Halo Profile}

In a cosmological simulation based on the comoving SP equations, 29] found that FDM halos contain distinct solitonic cores embedded in an NFW-like profile. We can approximate the density profile of FDM halos by

$$
\rho_{\mathrm{FDM}}(r)=\Theta\left(r_{\epsilon}-r\right) \rho_{c}(r)+\Theta\left(r-r_{\epsilon}\right) \rho_{\mathrm{NFW}}(r),
$$

where $\Theta$ is the Heaviside step function, $r_{\epsilon}$ is the transition radius where the density profile changes from cored profile to NFW-like behaviour, and the soliton density can be well described by [29, 4 ]

$$
\rho_{c}(r)=\frac{1.9 a^{-1}\left(m_{a} / 10^{-23} \mathrm{eV}\right)^{-2}\left(a^{-1} r_{c} / \mathrm{kpc}\right)^{-4}}{\left[1+9.1 \times 10^{-2}\left(r / r_{c}\right)^{2}\right]^{8}} M_{\odot} \mathrm{pc}^{-3} .
$$


Defining the core mass $M_{c}$ as the mass enclosed within $r_{c}$, [4] found a relation between the core mass and the halo mass,

$$
M_{c}=\frac{1}{4} a^{-1 / 2}\left[\frac{\Delta_{\mathrm{vir}}(z)}{\Delta_{\mathrm{vir}}(0)}\right]^{1 / 6}\left(\frac{M_{h}}{M_{\min , 0}}\right)^{1 / 3} M_{\min , 0},
$$

where $M_{\text {min }, 0}=375^{-1 / 4} \times 32 \pi \Delta_{\text {vir }}(0)^{1 / 4} \rho_{m 0}\left(H_{0} m_{a} / \hbar\right)^{-3 / 2} \Omega_{m 0}^{-3 / 4}$ is the minimum mass of FDM halos at the current time and $\Delta_{\text {vir }}(z)$ is the density contrast of a virialized halo. It should be noted that by definition, $M_{c}$ is at most $1 / 4$ of the halo mass. For a universe with a non-zero cosmological constant, $\Delta_{\text {vir }}(z)$ is given by [134]

$$
\Delta_{\mathrm{vir}}(z)=\frac{1}{\Omega_{m}(z)}\left[18 \pi^{2}+82\left(\Omega_{m}(z)-1\right)-39\left(\Omega_{m}(z)-1\right)^{2}\right] .
$$

Here $\Omega_{m}(z)$ is the matter fraction at the redshift $z$. For a matter dominated universe, $\Delta_{\text {vir }}=18 \pi^{2}$ just as we have derived before (see Eq. 2.60).

In actual computations, we do not implement the full density profile as in Eq. 2.103), because $r_{\epsilon}$ is still unknown (see the discussion in [31] 10. Instead, we model FDM halos with NFW density profiles with a modified concentration parameter and account for the presence of the core as part of the satellite model that will be described in Section Section 2.3.4. For models with mixed FDM and CDM, the halo density profiles are treated in the same way as for CDM and no cores are assumed.

The shape of the NFW profile is determined by the concentration parameter. halos collapsing earlier when the average density of the Universe was larger are more concentrated. As shown in Section Section 2.3.1, FDM halos collapse at a higher critical overdensity than their CDM counterparts. They form later than CDM halos with the same mass, thus they are less concentrated. This is analogous to a similar effect seen in WDM; to account for it, we estimate the concentration parameter for FDM using a fitting formula derived for WDM [135]:

$$
\frac{c_{\mathrm{FDM}}}{c_{\mathrm{CDM}}}=\left(1+\gamma_{1} \frac{M_{1 / 2}}{M}\right)^{-\gamma_{2}},
$$

where $\gamma_{1}=15, \gamma_{2}=0.3$, and $M_{1 / 2}$ is the half-mode mass defined according to the wavenumber at which the transfer function of FDM falls to one half of the transfer function for pure CDM. The concentration parameter for CDM in Eq. 2.107) is calculated according to [136]. In the absence of large FDM simulations for calibrating the parameters $\gamma_{1}$ and $\gamma_{2}$, we set them to the same value as for WDM [135]. It is, however, a plausible assumption that FDM behaves more like collisional matter than

\footnotetext{
${ }^{10}$ In a recent paper, [124] simulated multiple mergers of solitons and found $r_{\epsilon} \approx 3.5 r_{c}$.
} 
CDM as a consequence of quantum pressure, so that stripping may be more effective. In order to account for these uncertainties, we increased (decreased) the value of $\gamma_{2}$ by a factor of $2(1 / 2)$ and adjusted $\gamma_{1}$ to match the concentration parameter at the half-mode mass. This variation gives rise to only an insignificantly small difference in the subhalo mass function (SHMF). We also experimented with an alternative model for the concentration parameter based on [137, 138, again finding only a small effect. The apparent insensitivity of the SHMF to changes of the concentration parameter justifies our use of Eq. (2.107) with WDM parameters until more detailed FDM simulations become available.

\subsubsection{Satellite Model}

When constructing merger trees, we actually look backward in time to determine the merger history of the halos at lower redshifts. These merger trees yield information about hierarchical structure formation due to mergers, but does not contain any detailed information about the merger process. The next task of SAMs is to look at how galaxies populate and evolve with dark matter halos. The evolution of different components in the halos, baryons and dark matter, are traced forward in time, yielding the properties of galaxies that we can compare with observations. During this process, additional models are needed. When two halos encounter in the merger tree, they are not simply assumed to be merged immediately. Instead, the larger one is treated as the host and the smaller one will be its subhalo. Subhalos together with the satellite galaxies in their center are then evolved in the gravitational potential of the host until they dissolve. In this dissertation, I will concentrate on the evolution of dark matter (sub)halos. Hereafter, "satellite", unless specified otherwise, will refer to the subhalo rather than the satellite galaxy although they are closely related. At the end, we get additional information about a dark matter halo, i.e. its substructure. Searching for deviations in the halo substructure from the predictions of the CDM model provides a promising way to constrain dark matter models.

GALACTICUS contains several different implementations of models for the evolution of satellites. The "simple" implementation only assumes that the satellite orbits the host and its mass stays constant for some time $t_{\text {merger }}$. After that the satellite is considered to be fully dissolved. The merger time scale needs to be modeled and calibrated to match simulations. By default, GALACTICUS uses the fitting formula from [139] which is calibrated to CDM $N$-body simulations:

$$
t_{\text {merger }}=\frac{0.94 \epsilon^{0.60}+0.60}{0.86} \frac{M_{\text {host }}}{M_{\text {sat }}} \frac{1}{\ln \left(1+M_{\text {host }} / M_{\text {sat }}\right)} \frac{r_{\text {vir }}}{V_{\text {vir }}},
$$


where $\epsilon$ is a parameter that characterize the eccentricity of the orbit. $M_{\text {host }}$ and $M_{\text {sat }}$ are the masses of the host and satellite, respectively. $r_{\text {vir }}$ is the virial radius of the host and $V_{\text {vir }}$ is the circular velocity at $r_{\text {vir }}$. However, GALACTICUS also allows us to use several other formulas for the calculation of the merger time scale, e.g. the ones from [100, 105, 140, 141, 142].

The "orbiting" implementation presented in [123] is a more sophisticated model. It follows the mass loss of the satellites due to tidal stripping and tidal heating, and integrates the satellites' orbits including a submodel for dynamical friction to derive the actual merger time. Here, we briefly summarize the model ingredients (see [123] for more details).

If an object becomes a satellite, it is assigned an orbit, drawing the orbital parameters from PDFs that have been derived from $N$-body simulations. We use GALACTICUS' default choice for these PDFs (see [143, 144]). The dynamics of the satellite in its host's gravitational potential are then evaluated, adding an additional acceleration $\boldsymbol{a}_{\mathrm{df}}$ from the dynamical friction [145]:

$$
\boldsymbol{a}_{\mathrm{df}}=-4 \pi G^{2} \ln \Lambda M_{\mathrm{sat}} \rho_{\mathrm{host}}\left(r_{\mathrm{sat}}\right) \frac{\boldsymbol{V}_{\mathrm{sat}}}{V_{\mathrm{sat}}^{3}}\left[\operatorname{erf}(X)-\frac{2 X}{\sqrt{\pi}} \exp \left(-X^{2}\right)\right],
$$

where $M_{\text {sat }}$ is the mass of the satellite, $\rho_{\text {host }}\left(r_{\text {sat }}\right)$ is the density of the host halo at the distance of the satellite, $\boldsymbol{V}_{\text {sat }}$ is the velocity of the satellite, $X=V_{\text {sat }} / \sqrt{2} \sigma_{\mathrm{v}}$ with the velocity dispersion of the host $\sigma_{\mathrm{v}}$, and $\ln \Lambda$ is the Coulomb logarithm.

To account for tidal stripping, a tidal radius is defined so that all the mass outside the tidal radius is stripped from the satellite in one orbital period [146]:

$$
x_{\mathrm{t}}^{3}=\frac{G M_{\mathrm{sat}}\left(<x_{\mathrm{t}}\right)}{\omega^{2}-\mathrm{d}^{2} \Phi / \mathrm{d} r^{2}},
$$

with $M_{\text {sat }}\left(<x_{\mathrm{t}}\right)$ being the enclosed mass within the tidal radius, $\omega$ the angular velocity, and $\Phi$ the gravitational potential from the host. The mass loss rate due to tidal stripping is given by

$$
\dot{M}=\alpha \frac{M_{\text {sat }}\left(<x_{\mathrm{t}}\right)}{T_{\text {orbit }}},
$$

where $\alpha$ is a free parameter needed to be calibrate to simulations and $T_{\text {orbit }}$ is the orbital time of the satellite. In practice, the angular period and the radial period of the satellite are computed and $T_{\text {orbit }}$ is taken to be the larger one, i.e.

$$
T_{\text {orbit }}=2 \pi r_{\text {sat }} \max \left\{\frac{r_{\text {sat }}}{\boldsymbol{r}_{\text {sat }} \times \boldsymbol{V}}, \frac{r_{\text {sat }}}{\boldsymbol{r}_{\text {sat }} \cdot \boldsymbol{V}}\right\}
$$


Finally, when the satellite has a close encounter with the host, the tidal force changes on time scales much less than the characteristic dynamical time of the satellite, leading to a shock on the satellite. The satellite obtains extra dynamic energy after the encounter (shock), i.e. is heated up. This effect is called tidal heating. It expands the satellite and makes it more susceptible to tidal stripping. In GALACTICUS, this effect is modelled using the results by [147, 148].

In summary, as the satellite orbits the host, its orbital radius shrinks over time due to dynamical friction and its bound mass decreases gradually due to tidal stripping. Eventually it is fully dissolved in the host. Several criteria are used for terminating the evolution:

(1) the distance to the host is lower than the sum of the half-mass radius of both satellite and host;

(2) the distance to the host becomes less than 1 percent of the host's virial radius;

(3) the bound mass of the satellite is less than some fraction (1 percent by default) of its initial mass when becoming a satellite.

If either one of the above criteria is satisfied, the satellite will be assumed to be fully merged with the host.

\subsubsection{Modifications for FDM}

The existence of a core in the halo is a generic property of FDM models, changing the dark matter halo profile. While we expect these changes not to be significant at large radii, the close interaction of satellites and hosts with each other may potentially depend on the profile. In order to estimate the range of potentially observable effects, we use the satellite model to test several model assumptions, motivated in part by [62] who ran detailed simulations of merging solitonic cores.

As a first assumption, we set the tidal stripping rate to zero once the bound mass of the satellite approaches $4 M_{\mathrm{c}}$ (the total mass of an FDM halo when it is dominated by the solitonic core), representing the idea that the compact core of the satellite is stable against tidal disruption. We will revisit this assumption in Chapter 3. Secondly, we change the merging criteria. Host and satellite merge if the distance between them is smaller than the sum of their core radii, or if the satellite has less than 1 percent of its initial bound mass left. The criterion for the core radius is motivated by the observation that merging events happen rapidly once the cores touch [62]. Physically, this is expected only to be relevant for small halos that have relatively dominant cores 
while in more massive objects, one would expect the dynamics to be governed by the extended halo rather than the core. However, for these massive halos the bound mass criterion will apply before the cores interact, yielding only mild changes in the SHMF at high masses (see Section 2.4.3).

Additionally, the core mass is assigned along the merger history. First, we assign a core mass to all the halos in a tree that have no progenitors according to the core mass-halo mass relation found in [4], i.e. Eq. (2.105). Then the core mass may be changed by merger events. Minor mergers, defined as mergers with core mass ratios higher than $7 / 3$, do not affect the core mass of a halo. This is again motivated by [62] who showed that at high core mass ratios, the core of the smaller halo is simply disrupted and does not add mass to the solitonic core. For major mergers (mergers with mass ratios smaller than $7 / 3$ ), we set the core mass of the descendant halo $M_{c}$ given the core masses of the progenitors, $M_{c, i}$, to

$$
M_{c}=\beta \sum_{i} M_{c, i}
$$

where $\beta$ parametrizes the mass loss of the cores in a merging event. A value of $\beta=1$ corresponds to the case where the core masses are simply summed up. We use a value of $\beta=0.7$ as found by [62].

As a last modification, we disable the tidal heating in the model. It is unclear how tidal heating would affect an FDM profile that has a core. In particular, the core itself should not expand at all. Therefore, we simply ignore tidal heating.

We note that we do not modify other parts of the satellite model. For instance, we use distributions of the orbital parameters of satellites calibrated from CDM simulations. Obtaining these distributions for FDM will require large cosmological simulations that are not available to date. In this work, we broadly explore the possible effects of FDM cores on these scales. A complete, self-consistent model is beyond the scope of this thesis.

\subsection{Results for (Sub)halo Mass Function from SAMs}

The GALACTICUS code v0.9.4 (revision c49f04858120) is used with the modifications explained above (Section 2.3). We compare the results for different FDM density fractions $\Omega_{a} / \Omega_{d}$ and different FDM masses $m_{\mathrm{a}}$ with the standard CDM model. The cosmological parameters are taken from Planck 2015 [77]: $\Omega_{\mathrm{m}}=0.306, \Omega_{\Lambda}=0.694$, $h=0.6781, n_{\mathrm{s}}=0.9677$, and $\sigma_{8}=0.8149$. 

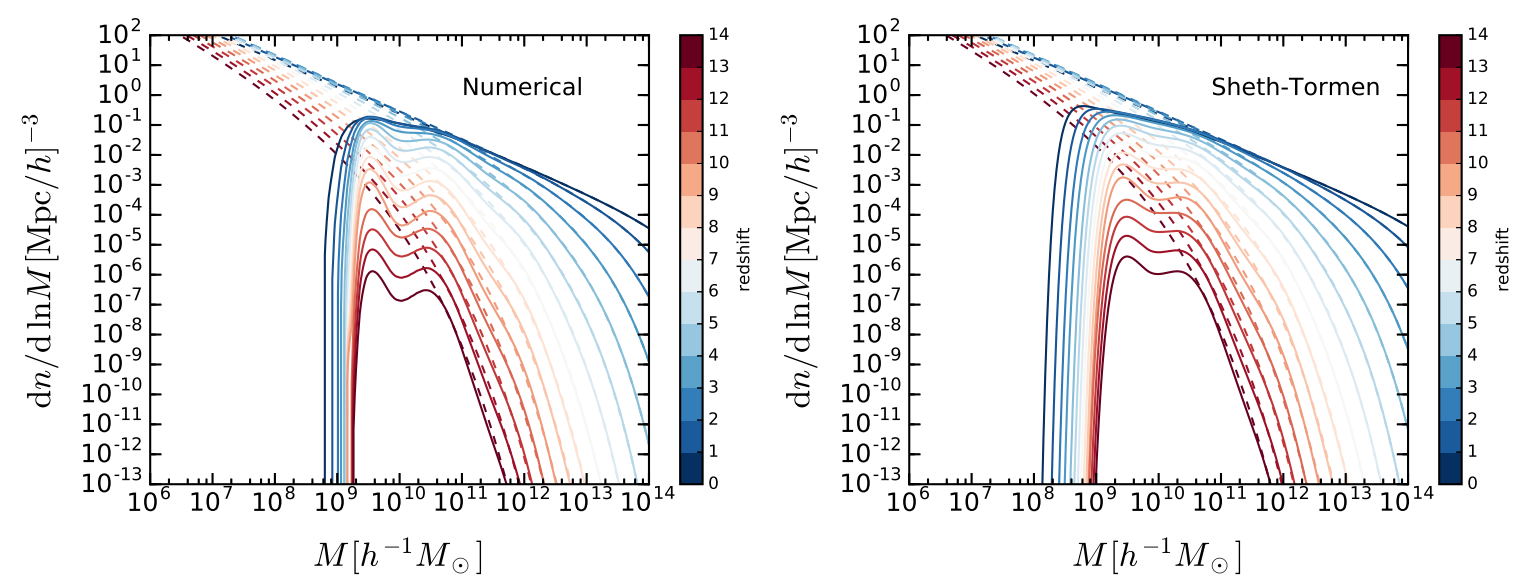

Figure 2.10: HMF for FDM fraction $f=1.0$ (solid lines) compared to standard CDM (dashed lines). Redshifts range from $z=14$ to 0 , obtained using our new calculation detailed above (left panel) and the Sheth-Tormen formalism (right panel). The FDM mass is set to $m_{\mathrm{a}}=10^{-22} \mathrm{eV}$. Reproduced from [1], Fig. 3.

\subsubsection{Halo Mass Function}

As discussed in Section 2.3.1, we solve the excursion set problems numerically for FDM with a redefined critical overdensity for collapse for FDM. The HMF we obtain for pure FDM with $m_{\mathrm{a}}=10^{-22} \mathrm{eV}$ is shown in Fig. 2.10 (left panel), with each line showing the HMF at a different redshift (see legend). We also show in the right panel the FDM HMF as derived in previous investigations [32] by simply replacing the critical overdensity in Eq. 2.97) with the one for FDM. Here the critical overdensity for collapse before remapping is computed using numerical results from AxionCAMB.

As we can see from Fig. 2.10, left panel, the HMF of FDM we obtain numerically shows the characteristic cutoff below a redshift-dependent minimum mass owing to quantum pressure. Compared with the ST formalism (right panel), we obtain a higher cutoff. At $z=0$, the cutoff mass is about $6 \times 10^{8} h^{-1} M_{\odot}$, roughly four times the value obtained from the ST formalism. Additionally, the cutoff mass of the HMF changes less strongly with redshift. At $z=14$, the cutoff mass is $2 \times 10^{9} h^{-1} M_{\odot}$, only two times of the value obtained from the ST formalism.

Next, we consider the case that DM is composed of a mixture of CDM and FDM (where the CDM component may simply be another ULA species with higher mass). We fix the total matter density of DM and change the fraction of FDM, $\Omega_{a} / \Omega_{d}$, from 0 (pure CDM) to 1 (pure FDM). The HMFs at $z=0$ for different fractions is shown in Fig. 2.11. We find that on large scales, the HMFs for MDM models are consistent with CDM as expected. However, on small scales the HMF is suppressed. 


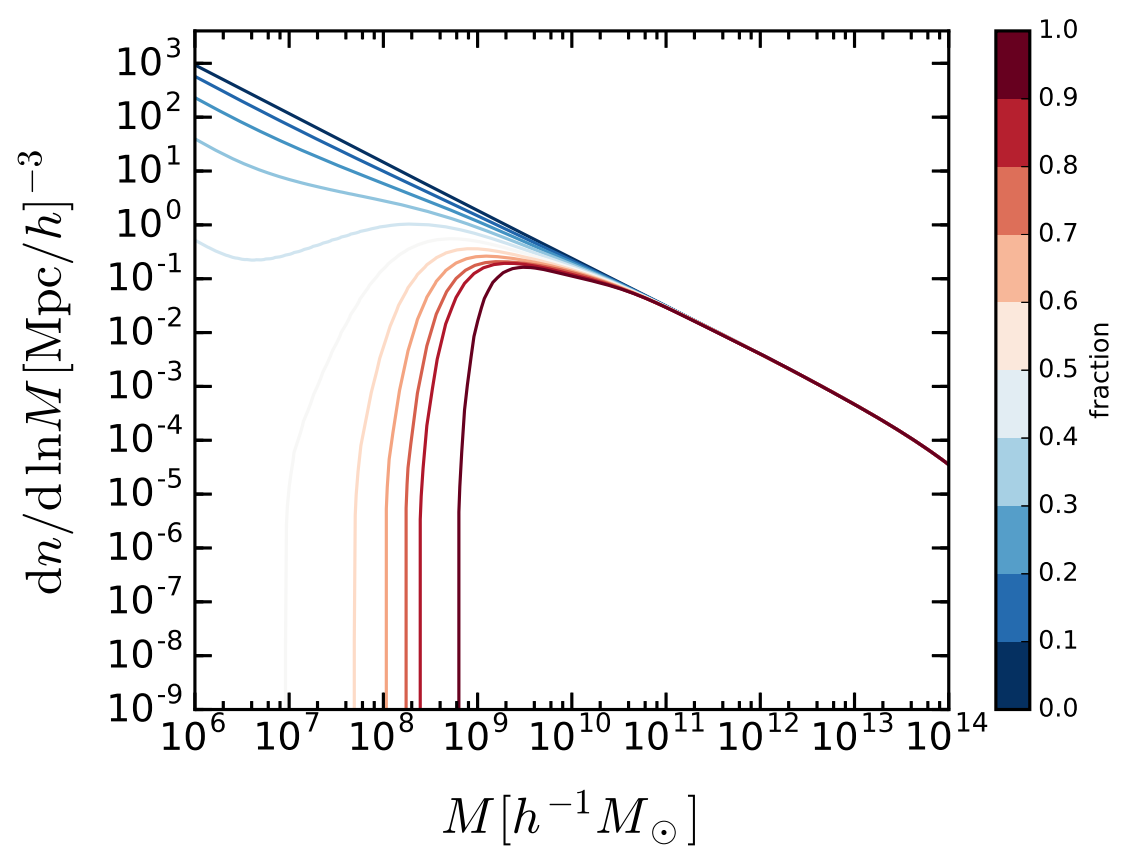

Figure 2.11: HMF at $z=0$ with different FDM fractions $f$. The fractions range from 0 to 1 with a step size of 0.1 . The FDM mass is set to $m_{\mathrm{a}}=10^{-22} \mathrm{eV}$. Reproduced from [1], Fig. 4.

With increasing $\Omega_{a} / \Omega_{d}$, the suppression becomes more and more significant. Around equipartition between $\mathrm{CDM}$ and $\operatorname{FDM}\left(\Omega_{a} / \Omega_{d} \sim 0.5\right)$, we recover a sharp cutoff as in the pure FDM case, albeit at lower cutoff masses $\left(\sim 10^{7} h^{-1} M_{\odot}\right)$. For higher $\Omega_{a} / \Omega_{d}$, the cutoff mass increases. All of these results are, mostly by construction, qualitatively consistent with [55].

Finally, we also consider different particle masses for FDM, using fitting functions for the transfer function, Eq. (2.88) in [25], and $\mathcal{G}$, Eq. (2.93) [2]. The results are shown in Fig. 2.12. We find the cutoff in the HMF changing with FDM mass as expected. The smaller the FDM mass, the larger the cutoff; e.g. for $m_{a}=10^{-24} \mathrm{eV}$, the cutoff mass is about $10^{12} M_{\odot}$, a possibility clearly ruled out by the existence of Milky Way-sized halos.

Note that [99] use a sharp- $k$ filter to derive the EPS HMF. In this case, there is no clear definition of mass corresponding to the filter scale (see the discussion in Section 2.1.6. Thus, as is usually done in the CDM case, we solve the excursion set problem assuming the sharp- $k$ filter ${ }^{11}$, but use the top-hap filter when converting the

\footnotetext{
${ }^{11}$ If a different filter, e.g. a top-hap filter in the space or a Gaussian filter is used, when we change the filter scale, unlike in the case of a sharp-k filter [see Eq. (2.74)], the change of the smoothed overdensity will not be a Markov processes. Thus the equation Eq. (2.86) will be very different from what we have used (see e.g.[149]).
} 


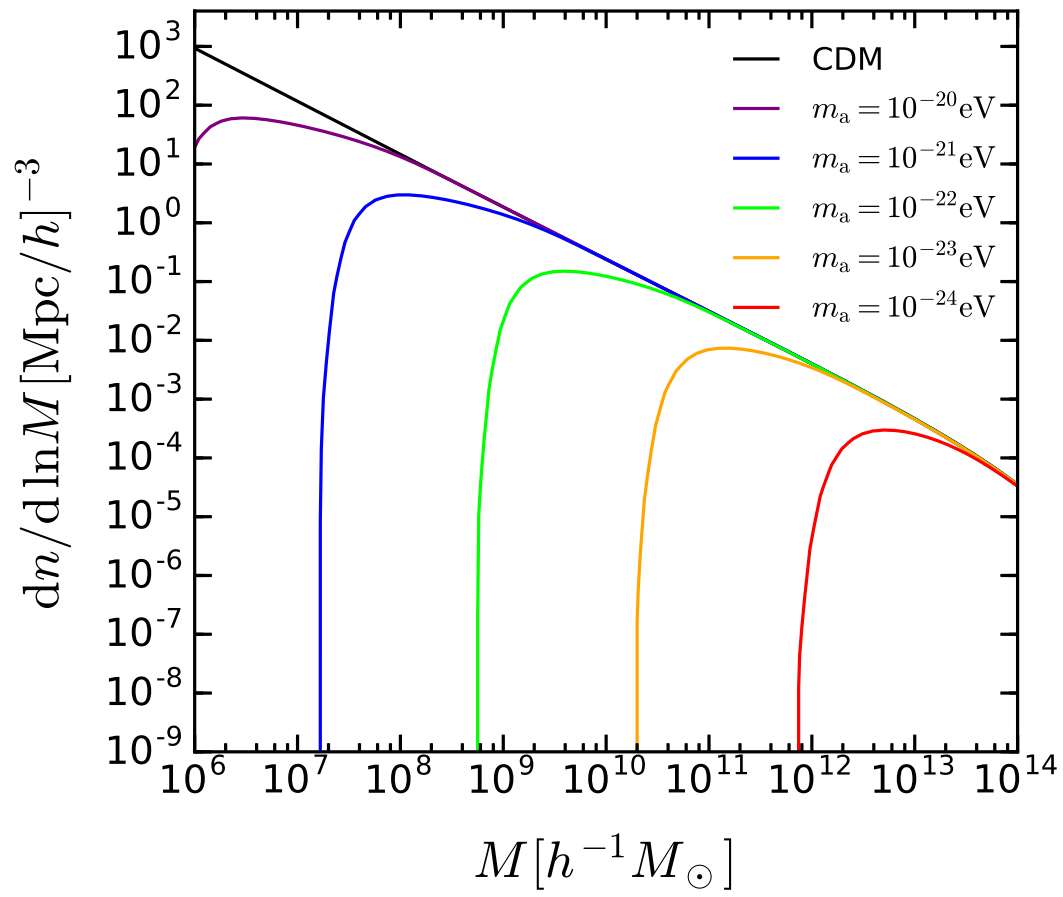

Figure 2.12: HMF at $z=0$ with different FDM masses compared to standard CDM. Reproduced from [1], Fig. 5.

variable $S$ (variance of the smoothed oversensity field) to halo mass (see Eq. (2.67)). Further discussions on different choices of window function for DM models with a suppressed power spectrum on small scales can be found in [101] and [102.

To test how accurate the HMF we derive is, we compare our result to the fitting formula found by [33]:

$$
\left.\frac{d n}{d \ln M}\right|_{\mathrm{FDM}}=\left.\frac{d n}{d \ln M}\right|_{\mathrm{CDM}}\left[1+\left(\frac{M_{h}}{M_{0}}\right)^{-1.1}\right]^{-2.2}
$$

where $M_{0}=1.6 \times 10^{10} m_{22}^{-4 / 3} M_{\odot}$. In order to get this formula, 33] perform Nbody simulations with FDM initial conditions, i.e. the quantum pressure during the nonlinear collapse is neglected.

Fig. 2.13 shows the HMF at $z=0$ for pure FDM with $m_{a}=10^{-22} \mathrm{eV}$ we obtained (solid black line) by solving the excursion set problems numerically compared to the prediction of the fitting function Eq. (2.114) (thick dashed line). For comparison, we also show the ST HMF for both CDM (thin dashed line) and FDM (dotted line). The orange line presents the HMF for FDM computed from another model proposed in [102, i.e. using a sharp-k filter in the ST formula Eq. (2.97) with the parameter $q=1$ 


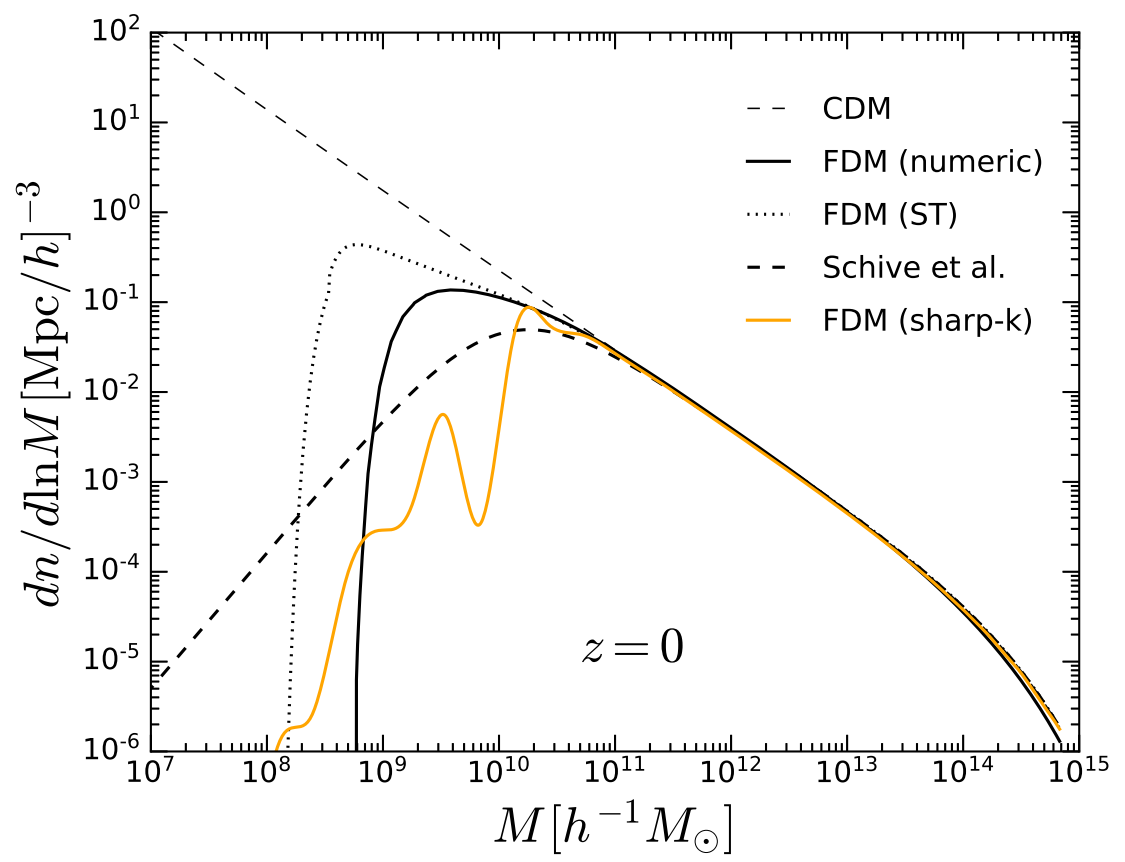

Figure 2.13: Halo mass function for FDM computed from different approaches compared to CDM.

(the critical overdensity for collapse is taken to be the same as in the CDM model). As can be seen, the ST HMF for FDM predicts too many small halos compared to the fitting formula. The sharp-k model can roughly capture the suppression scale of the HMF but underestimates the number of small halos. The HMF we obtained is close to the ST HMF at large and medium masses but drops off at a higher mass, thus is slightly more consistent with the fitting formula ${ }^{12}$. None of the models can reproduce perfectly the behavior of HMF seen in the simulations by [33. However, we should note that in their simulations, the quantum pressure during the nonlinear collapse is not accounted thus the abundance of the smallest halos which are most affected by this effect is not accurate.

In [3], the fitting function Eq. (2.114) is used to compute the cumulative HMF for FDM and to compare with the observed abundance of ultra-faint lensed galaxies in the Hubble Frontier Fields. Since the HMF for FDM drops off at lower masses, the cumulative number density of halos above $M_{\text {min }}$

$$
n\left(M>M_{\min }\right)=\int_{M_{\min }}^{\infty} M \frac{d n}{d \ln M} d M
$$

\footnotetext{
${ }^{12}$ This may change at very high redshifts when the HMF we obtain is always below the prediction of the fitting formula Eq. 2.114.
} 


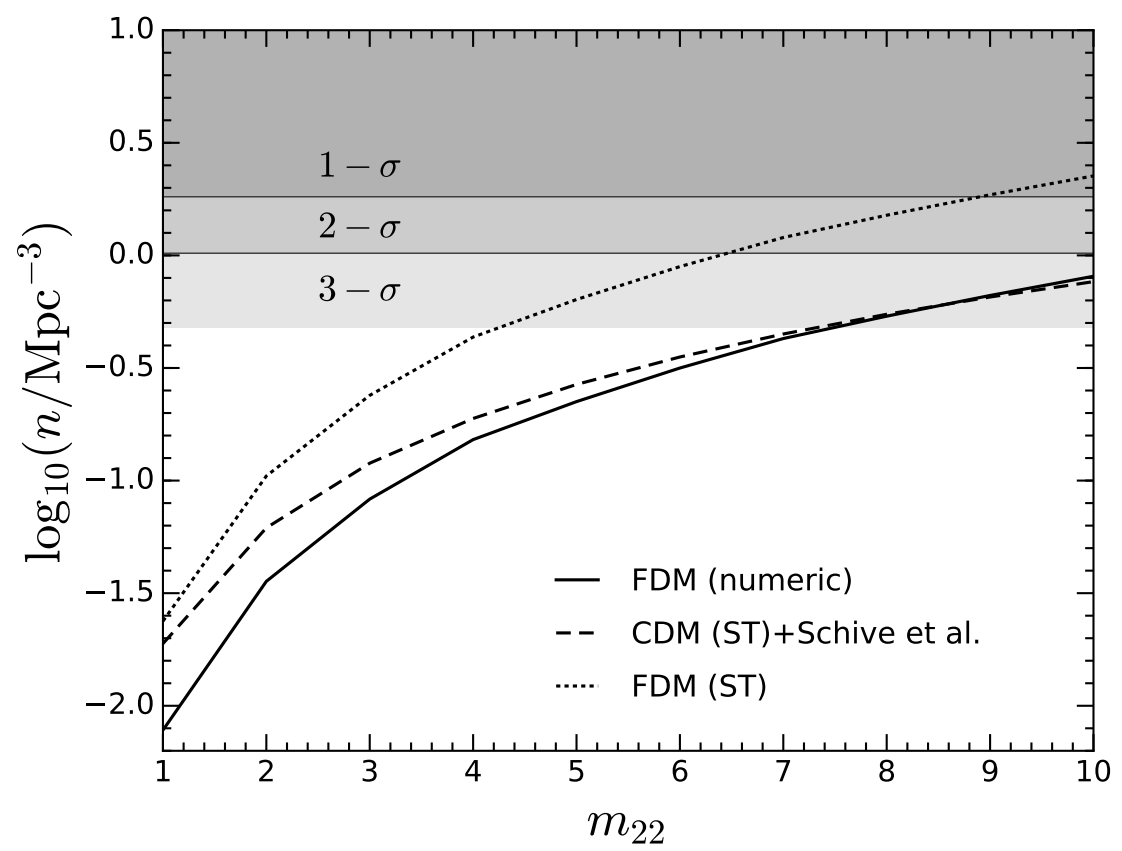

Figure 2.14: Cumulative number density of halos with $M>10^{6} M_{\odot}$ at $z=6$ calculated from different HMFs compared to observation [3]. $m_{22} \equiv m_{a} /\left(10^{-22} \mathrm{eV}\right)$.

saturates at a maximum value when $M_{\min }$ approaches 0 . Thus the number of galaxies observed puts a lower limit on $n\left(M>M_{\min }\right)$ with $M_{\min } \rightarrow 0$. In Fig. 2.14, we show the cumulative number density of halos with $M>10^{6} M_{\odot}$ at $z=6$ for different FDM masses computed from the HMF we obtained numerically (solid curve) compared to the $1-\sigma, 2-\sigma$ and $3-\sigma$ regions of the observed cumulative number density from [3]. We also show the cumulative number density computed from the fitting HMF by [33] and the ST HMF for FDM. Similar to previous conclusions, the ST HMF overestimates the number of halos thus gives a less strong constraint on the FDM mass. For smaller FDM masses, the cumulative number density we obtain has a larger deviation from the one obtained by considering the fitting HMF Eq. (2.114). But for FDM with $m_{a}>5 \times 10^{-22} \mathrm{eV}$, our results are very close to the one computed from Eq. 2.114). Thus we get a similar lower constraint of $7 \sim 8 \times 10^{-22} \mathrm{eV}$ on the FDM mass as in [3].

\subsubsection{Validating Merger Trees}

To check the consistency of the merger tree algorithm, we run merger trees with 1000 trees per decade in mass for halos with masses in the range $\left[4 \times 10^{8}, 4 \times 10^{13}\right] M_{\odot}$ at $z=0$. The mass resolution is set to $2 \times 10^{8} M_{\odot}$. 
We compare the HMF obtained by counting the halos in our merger trees with the one derived from solving the excursion set problems at different redshifts. The left panel of Fig. 2.15 shows the HMF at $z=0$ for pure FDM, $\Omega_{a} / \Omega_{d}=0.5$, and pure $\mathrm{CDM}$, with the symbols showing the data while the lines show the expected HMF from directly solving the excursion set problem as discussed in the previous sections. In order to show the points and error bars more clearly and avoid overlapping, the centers of bins are chosen slightly differently when counting the halos in merger trees. We can see that the HMFs obtained from merger trees can reproduce the excursion set problem solutions. The large errors at higher masses are due to relatively few massive halos.

Similarly, the right panel of Fig. 2.15 shows the HMF obtained from the merger trees compared to the direct solution for pure CDM and two different FDM masses. The agreement with the expectations is good.

The left (right) panel of Fig. 2.16 again shows the HMF from the merger trees for different FDM fractions $\Omega_{a} / \Omega_{d}$ ( FDM masses), but for a different redshift of $z=3$. We find an acceptable agreement, despite small deviations at intermediate halo masses. At even larger redshifts, the HMFs derived from the merger trees deviate more from the excursion set solutions. This is caused by small deviations in the branching modelling that accumulate with redshift, since the tree generation algorithm works "top to bottom", i.e. it starts at $z=0$ and generates progenitors. This deviation suggests that we may need to recalibrate the [133] modifications to the merger rate for FDM to get more accurate merger histories. Since there exist no sufficiently large cosmological simulations for FDM at present, we leave this to future work. We will show that this deviation at higher redshifts does not have a significant effect on the substructures of halos at lower redshifts.

\subsubsection{Subhalo Mass Function}

In Section 2.4.1, we have shown HMFs for FDM with different density fractions and masses from our new approach. In this section, we will use the satellite models described in Section 2.3.4 and the modifications presented in Section 2.3.5 to explore the subhalo mass function (SHMF).

We ran merger trees with 1000 trees per decade in mass for Milky Way-sized parent halos, $1 \times 10^{12}<M_{\text {host }}<3 \times 10^{12} M_{\odot}$. We chose a mass resolution of $M_{\text {res }}=$ $5 \times 10^{7} M_{\odot}$ and compared the results from two different satellite models, the "simple" implementation and the "orbiting" implementation. For the pure FDM case, we use the orbiting model with the modifications outlined above (see Section 2.3.5); for 

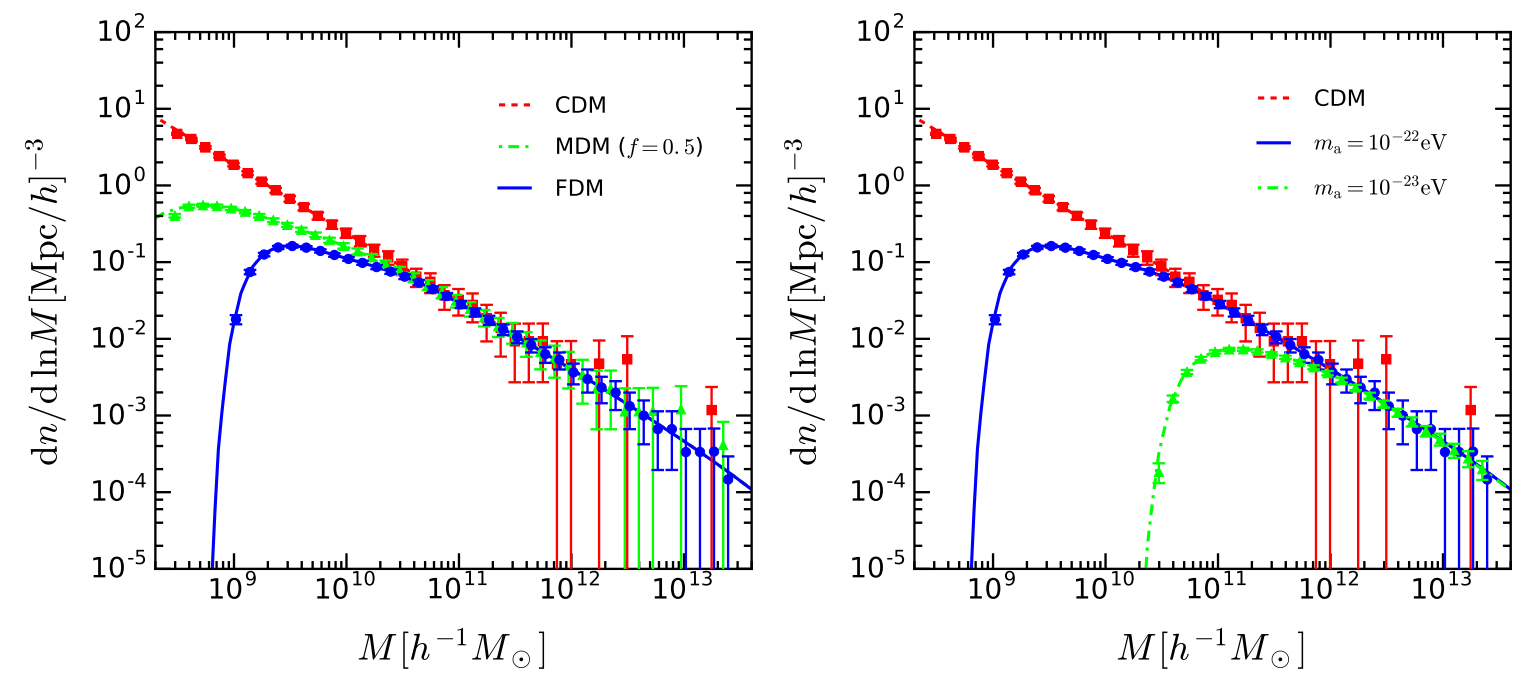

Figure 2.15: HMF from merger trees at $z=0$ for different models. Symbols indicate the data from the merger trees, the lines indicate the directly calculated HMF. Left: HMF from merger trees for pure FDM and MDM compared to standard CDM. The FDM mass is set to $m_{a}=10^{-22} \mathrm{eV}$. Right: HMF from merger trees for pure FDM with different masses compared to standard CDM. Reproduced from [1], Figs. 6 and 7 .
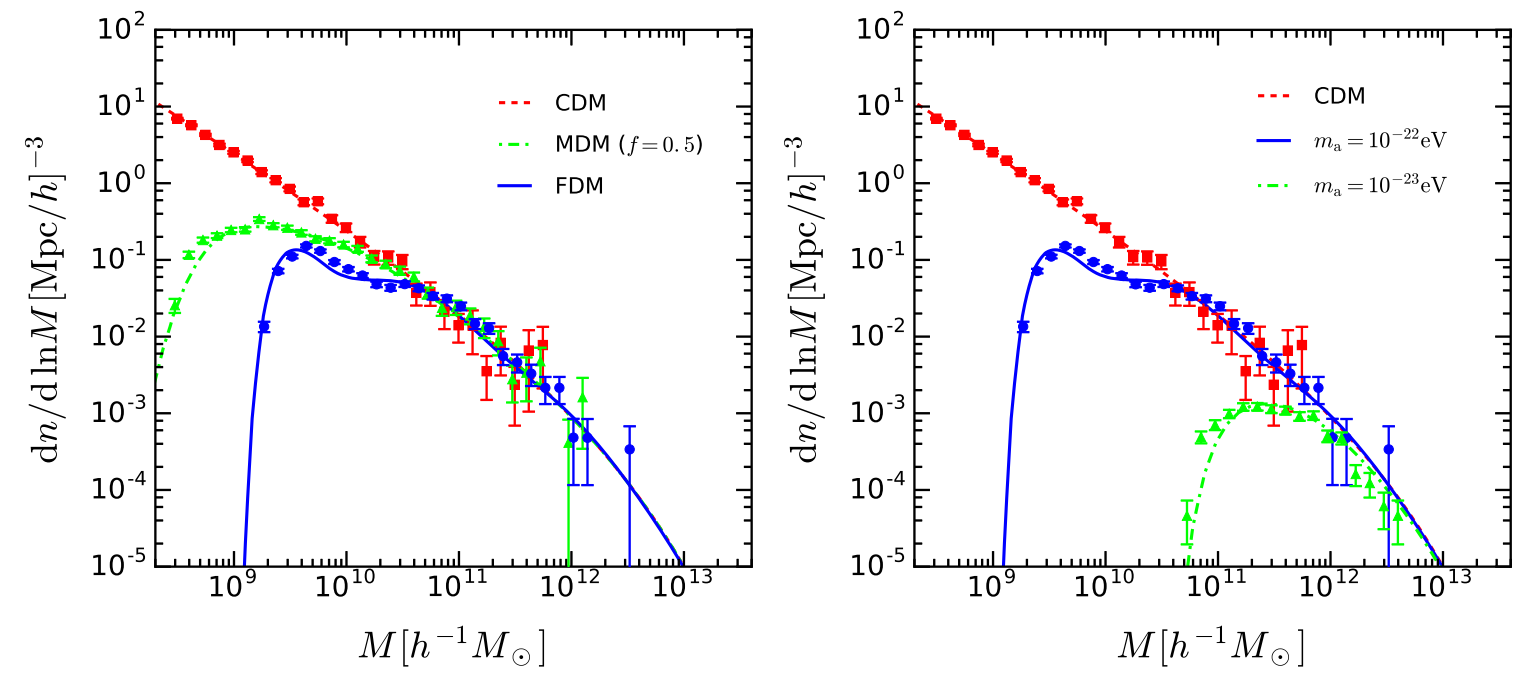

Figure 2.16: HMF from merger trees at $z=3$ for different models. Left: HMF from merger trees for pure FDM and MDM compared to standard CDM. The FDM mass is set to $m_{\mathrm{a}}=10^{-22} \mathrm{eV}$. Right: HMF from merger trees for pure FDM with different masses compared to standard CDM. Reproduced from [1], Figs. 8 and 9. 


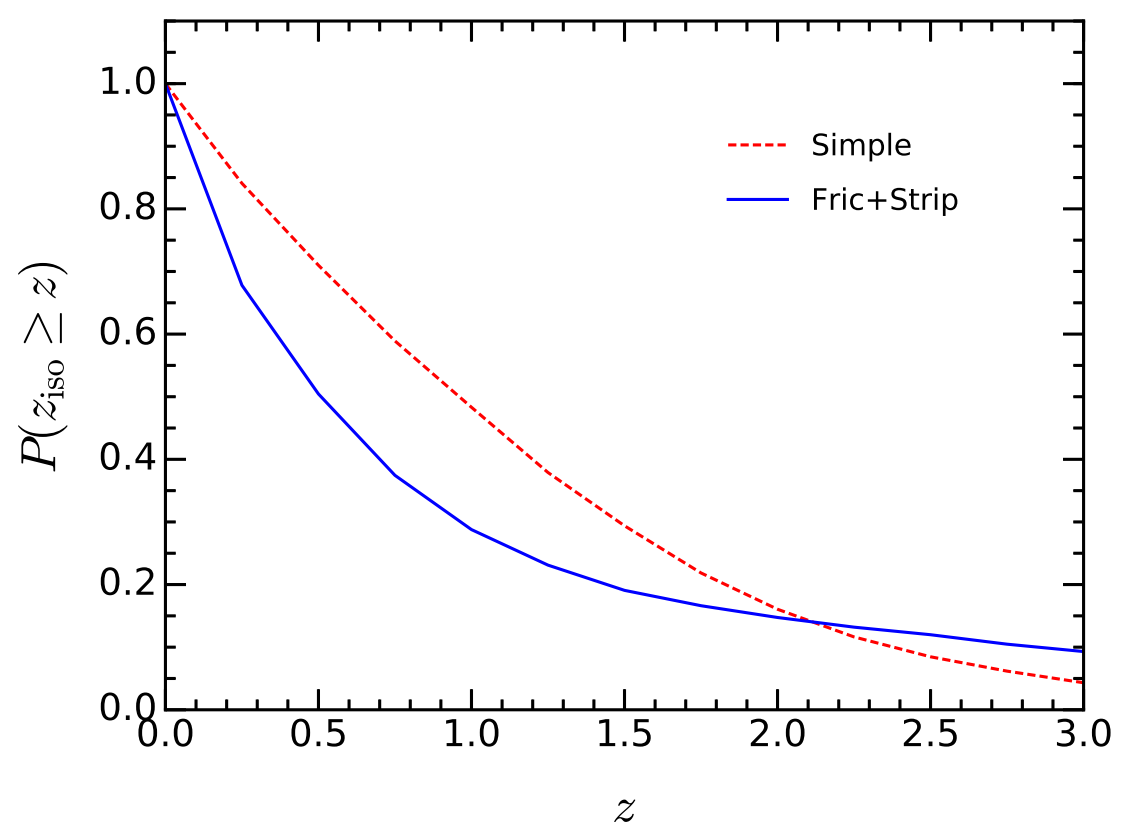

Figure 2.17: Cumulative distribution of the redshifts at which $z=0$ subhalos became subhalos for pure FDM $(f=1)$ with $m_{\mathrm{a}}=10^{-22} \mathrm{eV}$. Reproduced from [1], Fig. 10.

$\mathrm{CDM}$ and MDM, we use the unmodified version. In the orbiting implementation, we use the same parameters as in [123]: the Coulomb logarithm $\ln \Lambda=2.0$ and the tidal stripping mass loss rate parameter $\alpha=2.5$. The model for tidal heating in the orbiting implementation is switched off since it is physically unclear how this is to be treated given that FDM halos are expected to have compact cores.

As discussed in last section, the HMF obtained from merger trees may deviate from the one obtained by solving the excursion set problems at redshift $z>3$. This indicates that the merger tree structure at high redshifts suffers from the uncertainty caused by an inaccurate calculation of merging rate. In order to check how significantly this affects the SHMF, we use the redshift at which subhalos were last isolated as a diagnostic. As long as most of the subhalos with $z_{\text {iso }}>3$ have been completely merged in their host at $z=0$ (and therefore are not present in the substructure of their hosts), the high-redshift HMF does not affect the SHMF at low redshift. In Fig. 2.17, we show the cumulative fraction of subhalos with last isolated redshift $z_{\text {iso }} \geq z$ for the case with $f=1$ and $m_{\mathrm{a}}=10^{-22} \mathrm{eV}$. As can be seen, less than 10 per cent of subhalos originate from isolated halos at redshifts larger than 3. Thus, the uncertainty at higher redshifts can only have a very small effect on the SHMF at $z=0$.

To show the effects of dynamical friction and tidal stripping in the orbiting model 


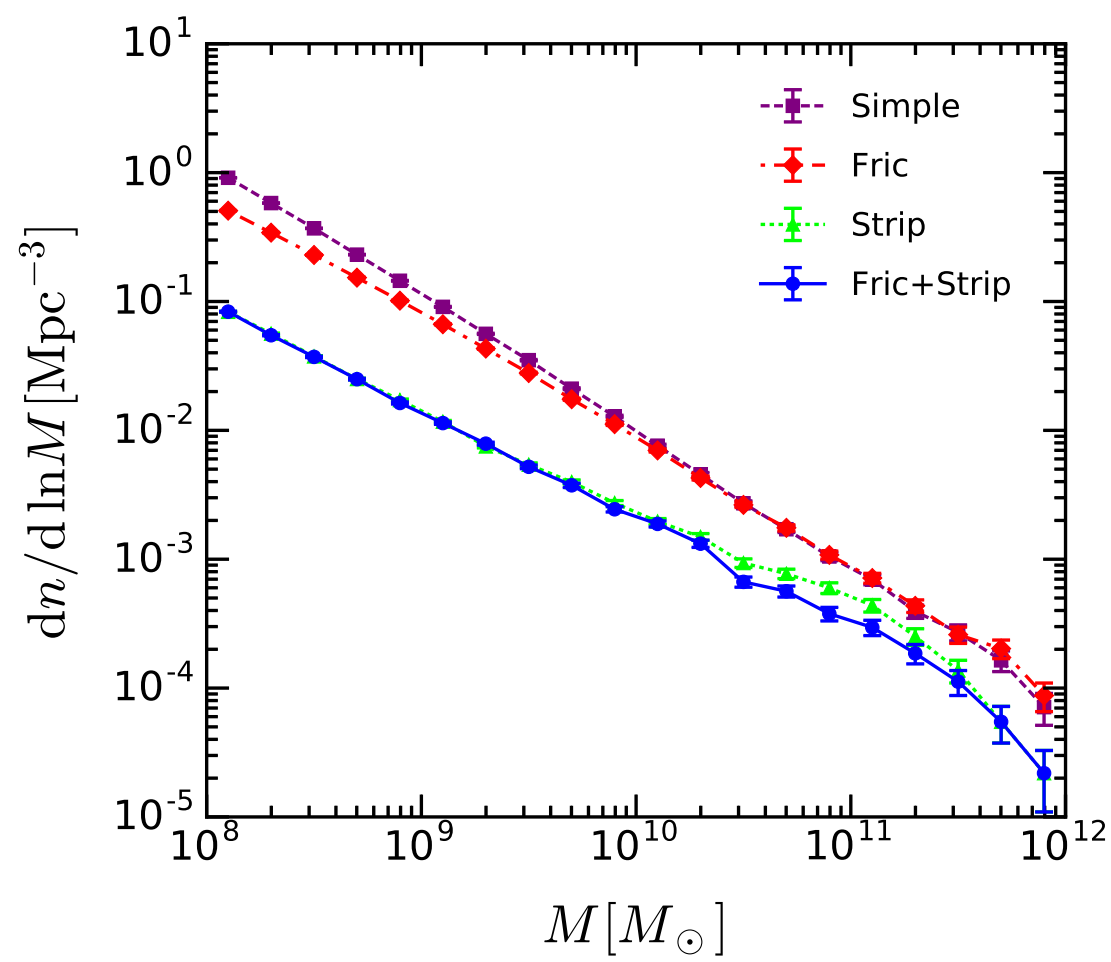

Figure 2.18: SHMF from merger trees for standard CDM. 'Fric' refers to dynamic friction and 'Strip' refers to tidal stripping. Reproduced from [1], Fig. 11.

separately, we show the SMHF with each effect switched on and compare them with the simple model. For the simple model, we use the merger time computed from [139], i.e. Eq. 2.108). We note that in the following, we use the concentration parameter as defined for CDM (see Section 2.3.3). We will analyze the influence of the concentration parameter on the stripping efficiency and therefore on the SMHF later. Fig. 2.18 shows the results for CDM, similar to Figs 2 and 3 in [123]. Due to changes between version v0.9.3 and v0.9.4 in GALACTICUS, there exist some small differences but the results are still comparable. We can see that if only dynamical friction is active, the SHMF is broadly consistent with the simple model. The tidal stripping effect reduces the amplitude of the SHMF. This is because the mass bound to the subhalos is gradually stripped by tidal forces, shifting the SHMF to the left which in turn makes the subhalos more vulnerable to further stripping. This is consistent with the results of [123].

Fig. 2.19 shows the SHMF for MDM with $\Omega_{a} / \Omega_{d}=0.5$ (left panel) and pure FDM (right panel). The FDM mass is taken to be $10^{-22} \mathrm{eV}$. For the MDM case, we use the original orbiting implementation, i.e. we do not include the effects arising from solitonic cores. As can be seen, if some fraction of dark matter is FDM, the SHMF 

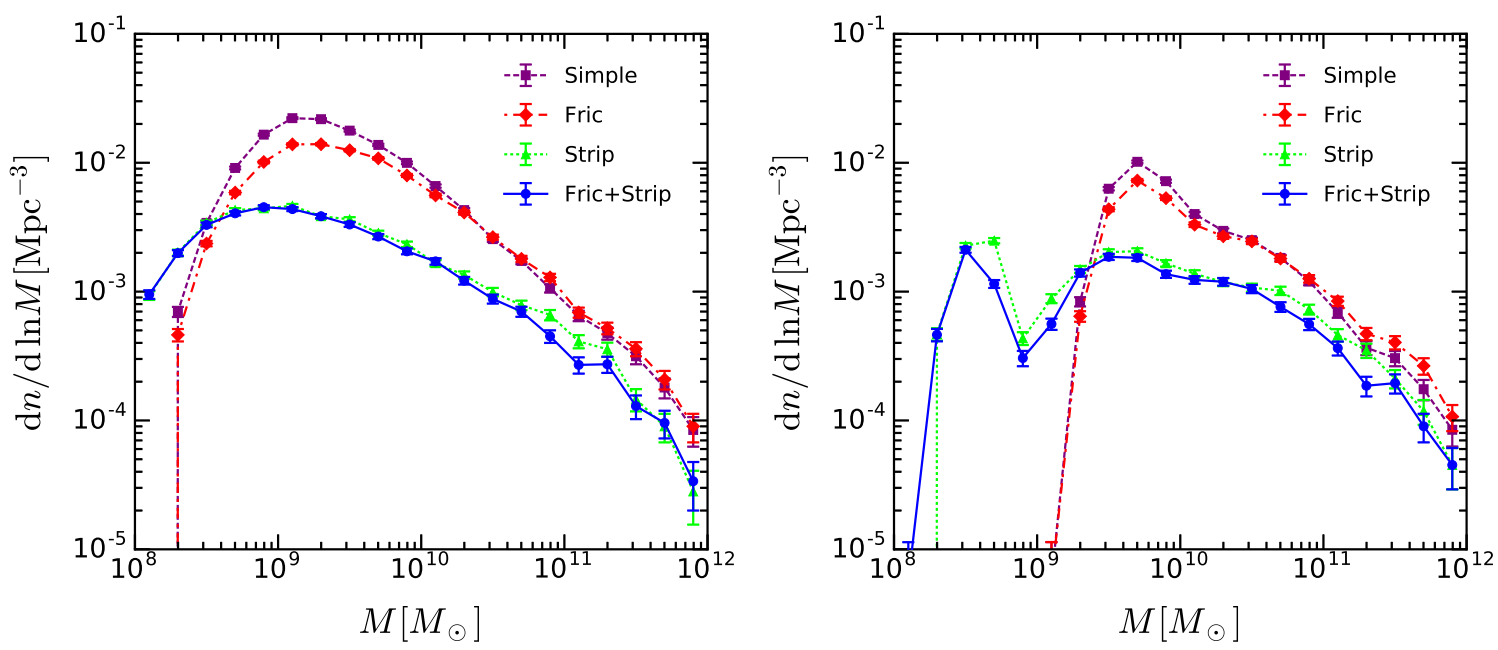

Figure 2.19: SHMF from merger trees for MDM with $\Omega_{a} / \Omega_{d}=0.5$ (left) and pure FDM. The FDM mass is set to $m_{\mathrm{a}}=10^{-22} \mathrm{eV}$. Modified from [1], Figs. 12 and 13.

is suppressed at lower masses.

In the simple satellite model and the orbiting model with only dynamical friction, subhalos do not lose mass until they dissolve within the host halo. In these models, we therefore observe a sharp cutoff in the SHMF at the HMF mass cutoff. In the more realistic orbiting model including tidal stripping, the subhalos will gradually lose their mass due to tidal stripping. This effect can be identified in Fig. 2.19; the SMHF obtained from the orbiting model including tidal stripping is shifted to the lower mass end. In particular, for the pure FDM case, we can see a bump that peaks at $3-4 \times 10^{8} M_{\odot}$ if we include tidal stripping in the orbiting model. This is expected as a consequence of our modification to stop tidal stripping at the solitonic core (cf. Section 2.3.4). These naked cores orbit their hosts until they satisfy one of the merging criteria, yielding a bump in the SMHF around $4 M_{\mathrm{c}}$ (the factor 4 follows from the definition of the core mass). At present, this feature is a direct consequence of our model assumptions. Large simulations resolving subhalo structure and dynamics are required to determine whether the feature in the SMHF is physical. In Chapter 3 , we will further discuss the evolution of solitonic cores in the host by doing idealized simulations.

The left panel of Fig. 2.20 shows a comparison of the SHMFs for FDM, MDM $\left(\Omega_{a} / \Omega_{d}=0.5\right)$, and CDM using the orbiting implementation with dynamical fraction and tidal stripping switched on. It can be seen that the SHMFs for FDM and MDM are suppressed at smaller masses compared to CDM, while at larger masses the three models are coincident. 

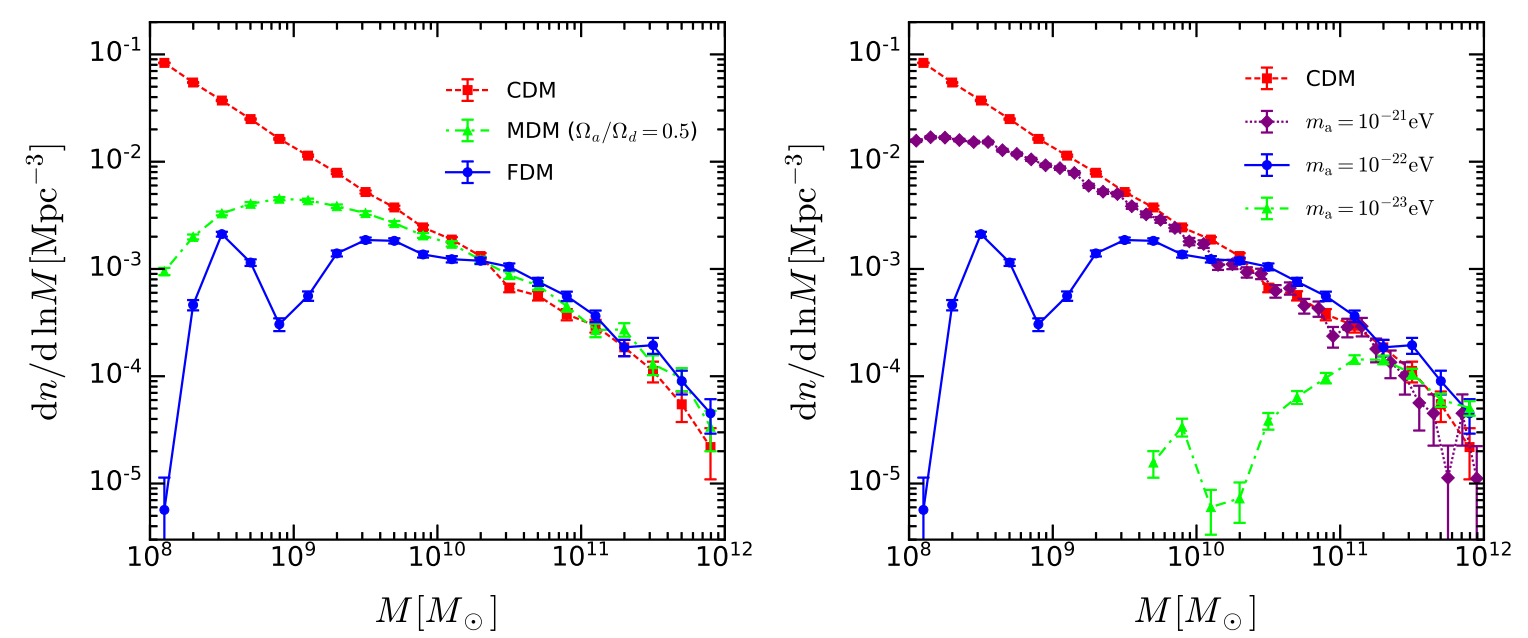

Figure 2.20: SHMF for FDM and MDM $(f=0.5)$ using the "orbiting" implementation with dynamical friction and tidal stripping compared with the standard CDM. The FDM mass is set to $m_{\mathrm{a}}=10^{-22} \mathrm{eV}$. Modified from [1], Figs. 14 and 15 .

We also compare the SHMF for FDM with different masses in the right panel of Fig. 2.20. For the case of $m_{\mathrm{a}}=10^{-23} \mathrm{eV}$, we ran the merger trees with 4000 trees per decade in mass in order to reduce the statistical errors close to the cutoff. With decreasing FDM mass, there are fewer lower mass subhalos as expected from the increased mass cutoff in the HMF for lower axion masses.

In Fig. 2.21, we show the results for SMHF for two different choices of the concentration parameter as described in Section 2.3.3. The differences are marginal. However, for the modified concentration parameter, the lower concentration parameter make halos more susceptible to tidal stripping. Consequently, the SMHF in the mass range $2 \times 10^{9} \sim 10^{11} M_{\odot}$ is slightly lower. 


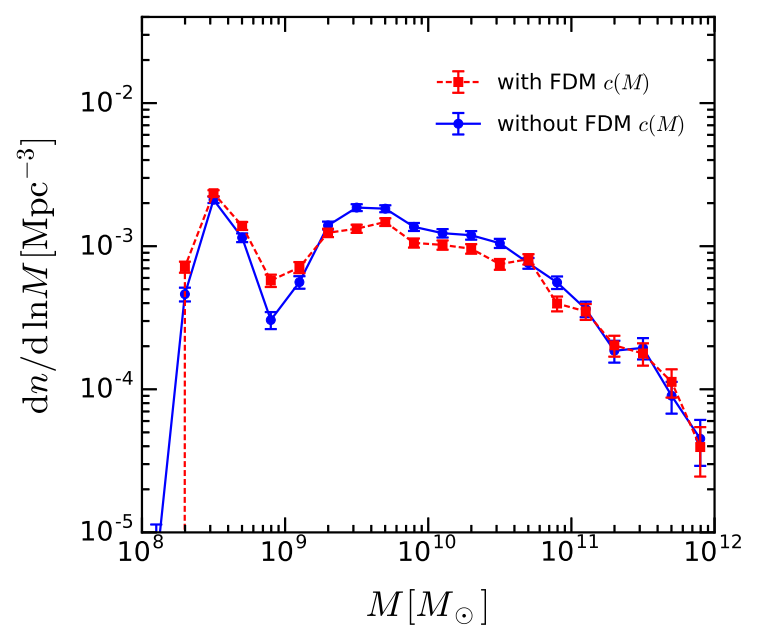

Figure 2.21: SHMF for FDM with and without modification to concentration parameter. The FDM mass is set to $m_{\mathrm{a}}=10^{-22} \mathrm{eV}$. Reproduced from [1], Fig. 16 . 


\subsection{Core Mass-Halo Mass Relation}

As mentioned before, simulations of cosmic structure formation [29] and merging solitonic solutions [4, 62, 124] based on the Schrödinger-Poisson (SP) equations indicate that FDM halos contain distinct cores surrounded by NFW-like profiles. [4] find that the mass of these cores, $M_{c}$, is related to the halo mass, $M_{h}$, by a power law relation, $M_{c} \propto M_{h}^{1 / 3}$ (see Eq. 2.105). They propose an explanation based on the relation

$$
M_{c}=\alpha(|E| / M)^{1 / 2},
$$

where $E$ is the total energy, $M$ is the total mass, and $\alpha$ is a constant of order unity, which they motivate heuristically with nonlocal consequences of the Heisenberg uncertainty relation. More specifically, the left-hand side $M_{c}$ is inverse to the core radius $r_{c}$, which can be easily checked by integrating the density profile of the solitonic core Eq. (2.104). The right-hand side represents the halo velocity dispersion $\sigma_{v}$. Thus Eq. (2.116) just indicates that $r_{c} \sigma_{v} \sim 1$, where we have ignored possible constants such as $\hbar$ and $m_{a}$. This looks very similar to the uncertainty relation. Identifying $M$

and $E$ with the virial mass $M_{h}$ and its energy $E_{h} \propto M_{h}^{2} / r_{\text {vir }} \propto M_{h}^{5 / 3}$ in Eq. 2.116, the numerically measured core-halo mass relation, $M_{c} \propto M_{h}^{1 / 3}$, can be recovered.

Note that the SP equations remain unchanged under the transformation $(r, M, E) \rightarrow$ $\left(r / \lambda, \lambda M, \lambda^{2} E\right)$ [150]. While Eq. 2.116) is consistent with this intrinsic scaling property, it is not unique (i.e., it can be multiplied by any scale invariant combination of $|E|$ and $M$, e.g. $\left.\left(|E| / M^{2}\right)^{\gamma}\right)$. Removing any residual effects of the scaling symmetry by constructing and analyzing scale invariant quantities, 62] were unable to reproduce this relation in simulations of solitonic core mergers (see also [124]). Furthermore, the model of [4] does not account for the combined evolution of $M_{c}$ and $M_{h}$ by halo mergers after the initial collapse of density perturbations which is known to be an important ingredient in hierarchical structure formation.

Comparing the initial and final masses of merging cores, [62] find a universal behavior of the core mass loss in mergers that depends nearly entirely on the mass ratio. As described in Section 2.3.5, we have implemented this feature in GALACTICUS, which enable us to track the core mass along the merger history.

In this section, we will further present a model for the core mass as a function of halo mass which is entirely based on the fractional core mass loss during major mergers. No further assumptions about the quantum nature of FDM halos and cores are necessary [5]. In particular, our model is independent of the dynamics of halo formation by gravitational collapse and hence insensitive to the initial core-halo mass relation of newly formed halos. 


\subsubsection{Simplified Model}

Given the mass loss fraction of cores during each merger, we can calculate the evolution of core mass along the merger history. Since cores merge and relax to their final state on a dynamical time scale once they begin to overlap [62], we only need to consider isolated binary mergers.

To calculate the evolution of core masses, we first need to know the initial core masses for halos without progenitors, i.e., those that form from direct collapse. Their mass is determined by the cutoff mass in the halo mass function (HMF), because the halos form hierarchically. As shown in [1], the cutoff mass depends only mildly on redshift, so the directly collapsed halos have approximately equal masses, $M_{\mathrm{h}, \min }$, independent of their collapse redshift. The initial core masses are therefore also expected to have roughly equal values, $M_{\mathrm{c}, \min }$.

Halo mergers change both core and halo masses. [62] find that only mergers with mass ratio $\mu<7 / 3$ yield an increased core mass $M_{c}=\beta\left(M_{c 1}+M_{c 2}\right)$. Here, $M_{c 1}$ and $M_{c 2}$ are the masses of the initial cores and $(1-\beta)$ is the core mass loss fraction, where $\beta \sim 0.7$ independent of the initial core masses. We refer to mergers with $\mu<7 / 3$ as major mergers. Larger mass ratios (minor mergers) leave the core mass of the more massive halo unchanged and result in the total disruption of the smaller halo. Smooth accretion corresponds to accretion with very high mass ratios and is treated in the same way.

In summary, in our model three types of physical interactions can change the core and halo masses: smooth accretion (see [1] for more details), minor mergers, and major mergers. The first two increase the mass of the halo but leave the core mass unchanged. Major mergers increase both halo and core masses.

Our model is based on a simplified description of the merging process. Suppose that $N$ halos with halo mass $M_{\mathrm{h} \text {,min }}$ and core mass $M_{\text {c,min }}$ merge to form one halo with mass $M_{h}$ whose contribution from mergers is $N M_{\mathrm{h}, \min }$. Assuming that the mass contributed by smooth accretion is also proportional to $N M_{\mathrm{h} \text {,min }}$, i.e. more massive halos accrete more mass from their surroundings, we have

$$
M_{h}=\alpha N M_{\mathrm{h}, \min }
$$

If the final halo encounters $N_{\text {major }}$ major mergers and $N_{\text {minor }}$ minor mergers, then

$$
N=N_{\text {major }}+N_{\text {minor }}+1
$$


The more major mergers the final halo encounters, the more minor mergers it also tends to have. Hence, we can assume

$$
N_{\text {minor }}=b(\beta) N_{\text {major }} \text {. }
$$

Here we have added the dependence on $\beta$ to the parameter $b$ since the definition of major (minor) merger is closely related to $\beta$. We will show below that the assumptions Eqs. 2.117) and (2.119) are reasonable. Given the mass of the final halo, Eqs. 2.117), 2.118) and (2.119) allow us to estimate the number of major mergers it has encountered:

$$
\begin{aligned}
N_{\text {major }} & =\frac{1}{1+b(\beta)}\left(\frac{M_{h}}{\alpha M_{\mathrm{h}, \text { min }}}-1\right) \\
& \approx \frac{1}{1+b(\beta)} \frac{M_{h}}{\alpha M_{\mathrm{h}, \text { min }}} .
\end{aligned}
$$

Since minor mergers do not change the core mass, we only need to consider major mergers when estimating the final core mass $M_{c}$. Suppose that during every major merger, both progenitors have the same core mass, i.e. the $\left(N_{\text {major }}+1\right)$ first-formed halos with core mass $M_{c, \text { min }}$ merge pairwise and form $\frac{N_{\text {major }}+1}{2}$ halos with core mass $2 \beta M_{\mathrm{c}, \min }$. This process continues until the formation of the final halo with mass $M_{h}$. The other $\left(N-N_{\text {major }}-1\right)$ first-formed halos are assumed to be accreted by minor mergers, thus they do not affect the core mass.

Fig. 2.22 shows a schematic diagram of the merger history of cores with the solid (dashed) line representing major (minor) merger and the dotted line representing smooth accretion.

As explained above, the first-formed halos have nearly identical core masses, hence the assumption that all major mergers have core mass ratio $\mu=1$ is reasonable for the first generation of merging events. As halos continue to merge, we overestimate the core mass because there will be major mergers with $\mu>1$ and correspondingly smaller core mass growth.

Finally, after $\log _{2}\left(N_{\text {major }}+1\right)$ generations of major merger events and $N_{\text {minor }}$ minor merger events, the final halo has a core mass of

$$
\begin{aligned}
M_{c} & =(2 \beta)^{\log _{2}\left(N_{\text {major }}+1\right)} M_{\mathrm{c}, \text { min }} \\
& =\left(N_{\text {major }}+1\right)^{\log _{2}(2 \beta)} M_{\mathrm{c}, \text { min }} \\
& \approx\left(N_{\text {major }}\right)^{\log _{2}(2 \beta)} M_{\mathrm{c}, \text { min }} .
\end{aligned}
$$




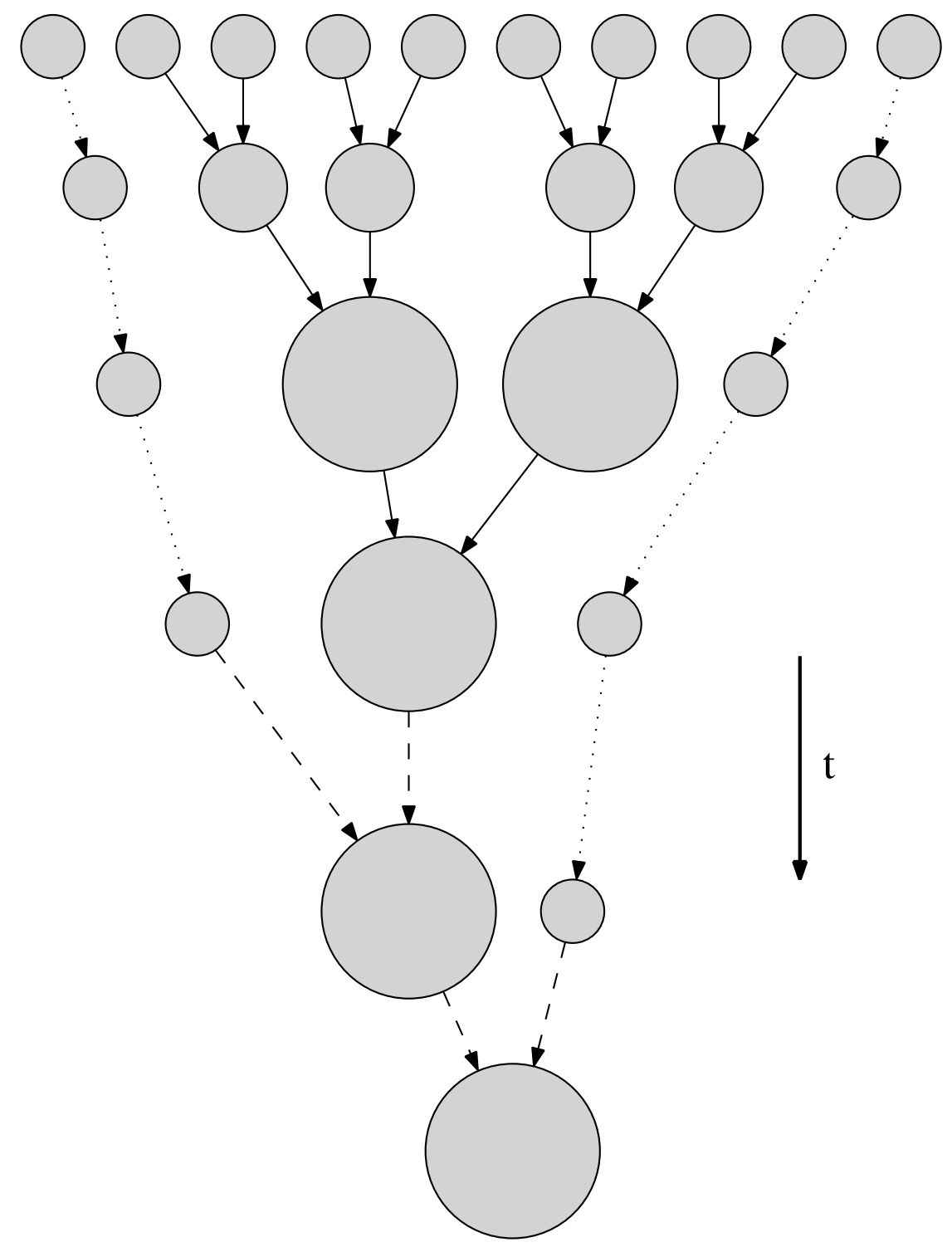

Figure 2.22: An example of a merger tree with 7 major mergers (solid lines) and 2 minor mergers (dashed lines). The dotted lines represent the smooth accretion. Note that the size of the circle represents the mass of the core (not necessarily in a consistent scale). 
Substituting Eq. 2.120 into Eq. 2.121), we have

$$
M_{c}=\left[\frac{1}{1+b(\beta)} \frac{M_{h}}{\alpha M_{\mathrm{h}, \min }}\right]^{\log _{2}(2 \beta)} M_{\mathrm{c}, \min } \equiv A M_{h}^{\log _{2}(2 \beta)} .
$$

Note that although the relation Eq. 2.122) does not explicitly depend on redshift, the prefactor $A$ does since $\alpha$ and $b$ may change with redshift. On the contrary, the exponent of $M_{h}$ only depends on the core mass loss fraction. In a binary merger, the core mass of the descendant will not be larger than the sum of core masses of its two progenitors, so $\beta \leq 1$ (e.g. 62] found $\beta \sim 0.7$ ). Treating $(2 \beta-1)$ as a small number, Eq. 2.122) yields

$$
M_{c} \propto M_{h}^{\log _{2}(2 \beta)} \approx M_{h}^{(2 \beta-1) / \ln 2} \approx M_{h}^{1.44(2 \beta-1)}
$$

to leading order. As discussed above, this relation overestimates the core mass when binary mergers with $\mu>1$ are involved. We will account for this effect below when comparing this prediction to our results from SAMs.

\subsubsection{Testing with Monte-Carlo Merger Trees}

In order to test the core-halo mass relation given in Eq. (2.122), we use the modifications to the SAM code GALACTICUS for FDM described in Section 2.3 and build 2000 merger trees for root halos with $4 \times 10^{11}<M_{h}<4 \times 10^{13} M_{\odot}$. The mass resolution is set to $2 \times 10^{8} M_{\odot}$. Without loss of generality, we set $m_{a}=10^{-22} \mathrm{eV}$. The parameter $\beta$ is set to 0.7 as found by [62] unless specified otherwise.

Fig. 2.23 shows an example of 15 trajectories of the core-halo mass relation chosen from the merger trees, i.e. each line shows the evolution of the core mass and halo mass along the main branch of one merger tree. The effects of smooth accretion, minor mergers, and major mergers can clearly be seen in this figure. Smooth accretion and minor mergers increase the halo mass while the core mass remains constant. Major mergers increase both the core mass and the halo mass.

Eq. 2.122 predicts that while the proportionality factor $A$ may depend on the initial core mass $M_{c, \text { min }}$, the exponent is independent of it.

To test the dependence of the core-halo mass relation on initial conditions, we implemented a power-law initial relation $M_{\mathrm{c}, \text { ini }} \propto M_{\mathrm{h}, \text { ini }}^{n}$ for halos that have no progenitors in the modified GALACTICUS code. Figure 2.24 shows the results for $n=1 / 3$, $n=1$, and $n=2$. Clearly, the core-halo mass relation at $z=0$ depends only very weakly on the initial mass distribution. 


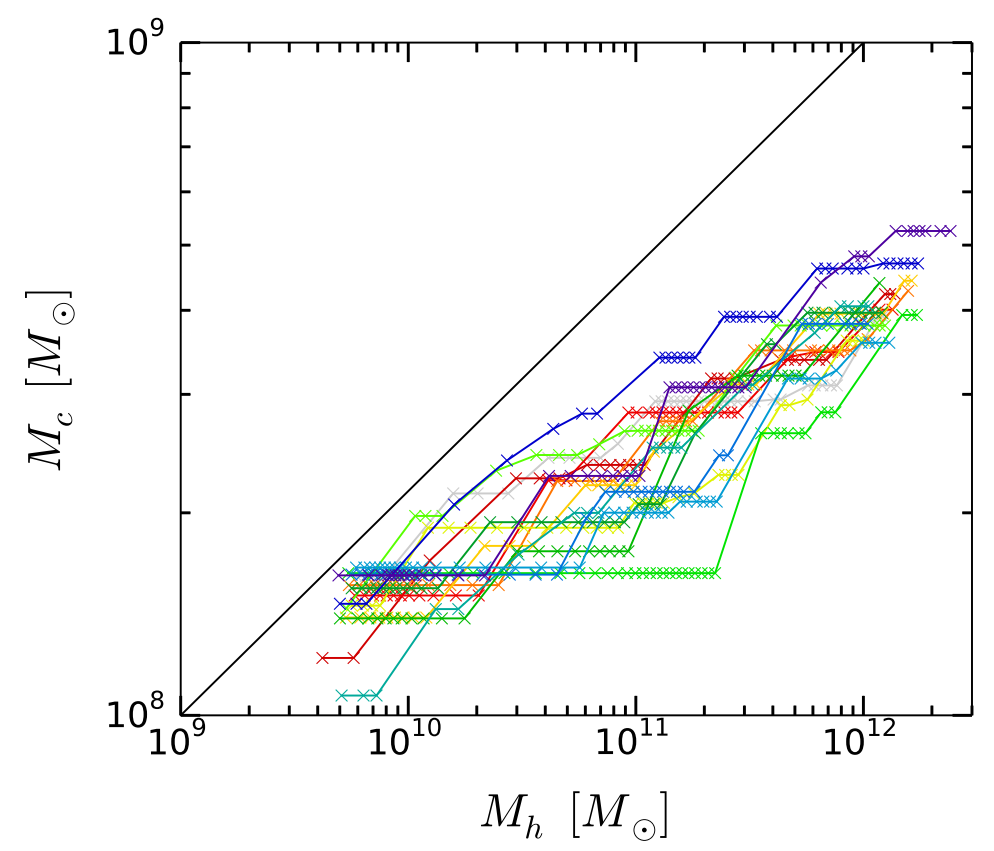

Figure 2.23: Evolution of core mass and halo mass for several merger trees. Only the main branch is shown. The straight line up-left shows the Schive et al. core-halo mass relation with arbitrary normalization as a reference.

In 4, the $1 / 3$ power-law relation between the core and halo mass is explained via the uncertainty principle. Although this approach may not be valid for halos which have encountered many mergers, it is applicable for halos that have just collapsed. Since there is no other preferred choice, we will use $n=1 / 3$ to set the initial core mass below. As is shown above, this specific choice does not have a significant effect on our results.

Next, we verify the two assumptions made in deriving Eq. 2.117) and Eq. 2.119). The left panel of Fig. 2.25 shows the halo mass $M_{h}$ with respect to the number of first-formed halos $N$ obtained from merger trees. Despite large scatter at small $N$ representing halos that have only encountered few mergers and are thus more strongly affected by the uncertainty of individual events, the assumed linear dependence Eq. (2.117) fits well.

The center panel of Fig. 2.25 shows the number of minor mergers $N_{\text {minor }}$ with respect to the number of major mergers $N_{\text {major }}$. Again, at small $N_{\text {major the data }}$ points have large scatter, but in general the assumption Eq. (2.119) gives a reasonable fit. Finally, the right panel of Fig. 2.25 shows the halo mass $M_{h}$ with respect to the number of major mergers $N_{\text {major }}$. This plot is a combination of the first two and is just meant to give a more relevant comparison between Eq. (2.120) inferred from the 

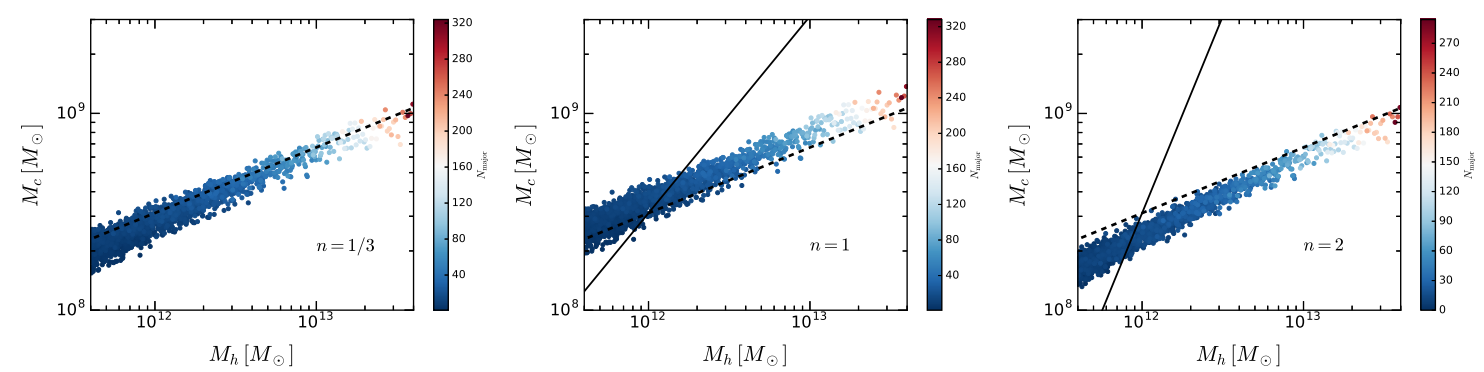

Figure 2.24: The core mass with respect to the halo mass at $z=0$ for different initial core-halo mass relation: $n=1 / 3$ (left), $n=1$ (center), and $n=2$ (right). The dashed line shows the core-halo mass relation from [4], $n=1 / 3$, at $z=0$. The solid lines show the linear and square relations for comparison. Reproduced from [5], Fig. 1.
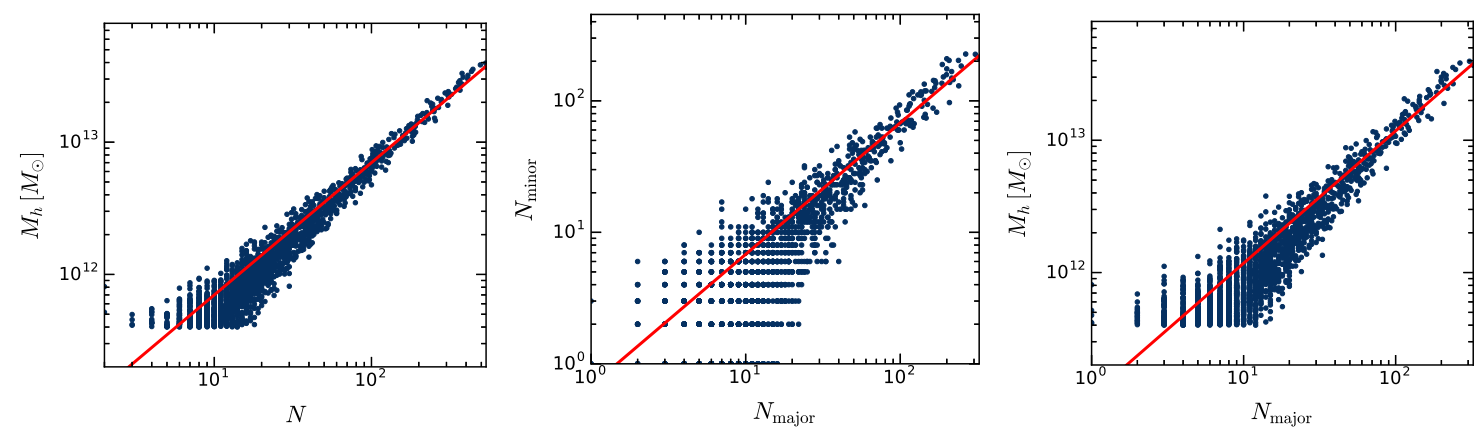

Figure 2.25: Left: the halo mass with respect to the number of first-formed halos. The solid line corresponds to Eq. 2.117). Center: the number of minor mergers with respect to the number of major mergers [solid line given by Eq. (2.119)]. Right: the halo mass with respect to the number of major mergers [solid line given by Eq. (2.120)]. Reproduced from [5], Fig. 2.

two assumptions and the results from merger trees.

To study the impact of the core mass loss fraction, we varied the value of parameter $\beta$ between 0.5 and 1. Correspondingly, we must also modify the definitions of minor and major merger: if the core mass ratio is larger (smaller) than $\beta /(1-\beta)$, the merger is defined as minor (major) merger. For $\beta=0.7$, we obtain the former definition.

Before showing the results from merger trees, we consider two extreme cases. In the case of $\beta=0.5$, the core mass does not change during any of the three possible interactions. The final core mass is solely determined by the initial core mass and independent of the final halo mass. On the contrary, for $\beta=1$, all mergers will be major mergers and the final core mass is given by $M_{c}=N M_{\mathrm{c}, \min }$. Since the halo mass is also proportional to $N$ [Eq. (2.117)], in this case the core-halo mass relation is linear. Expressed in the form $M_{c} \propto M_{h}^{\gamma(\beta)}$, we thus have $\gamma(0.5)=0$ and $\gamma(1)=1$. A simple linear parametrization for $\gamma(\beta)$ is $2 \beta-1$ which yields the core-halo mass 

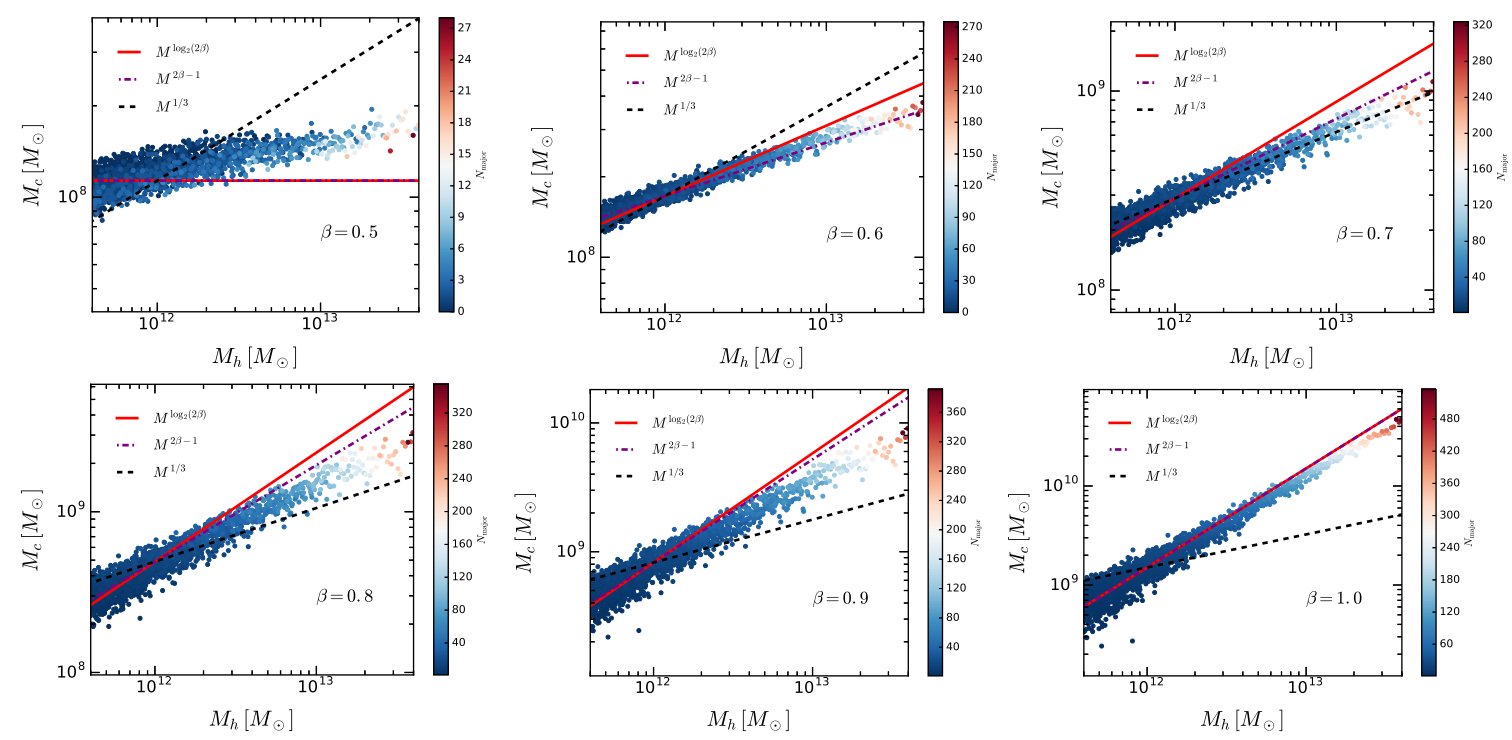

Figure 2.26: The core-halo mass relation at $z=0$ for different $\beta$ compared with predictions of different models. The three lines are matched at $M_{h}=10^{12} M_{\odot}$. Reproduced from [5], Fig. 3.

relation

$$
M_{c} \propto M_{h}^{2 \beta-1} .
$$

Note that it is very similar to Eq. 2.123) obtained from the merger history.

Figure 2.26 shows the core-halo mass relation at present time for different $\beta$ and compares them with the predictions from [4], Eq. (2.122), and the linear parameterization Eq. 2.124). Despite the simplifications in deriving Eq. 2.122), we find reasonable agreement for the core-halo mass relation for different core mass loss fractions $(1-\beta)$. At larger halo masses (implying more major mergers), the prediction of our model Eq. 2.122 tends to overestimate the core masses. Equation 2.124 gives a slightly better fit, implying that we can use it as a correction to Eq. (2.122). For $\beta=0.7$ [62], Eq. 2.124 yields $M_{c} \propto M_{h}^{0.4}$. It is close to the relation $M_{c} \propto M_{h}^{1 / 3}$ and fits the cosmological simulations [29] equally well.

In order to compare the core mass predicted for FDM halos with observations, the prefactor $A$ in Eq. 2.122 is also important. According to our results, we can replace $\log _{2}(2 \beta)$ in Eq. 2.122 with $2 \beta-1$ to give a better estimate of the core-halo mass relation. If we further assume that at the beginning, i.e. prior to any mergers, there were only pure solitons (instead of virialized halos produced by mergers of solitons), the initial core mass is $M_{\mathrm{c}, \min }=\frac{1}{4} M_{\mathrm{h} \text {,min }}$ by definition [29, 4]. Then we have

$$
M_{c}=\frac{1}{4} B\left(\frac{M_{h}}{M_{\mathrm{h}, \min }}\right)^{2 \beta-1} M_{\mathrm{h}, \min },
$$


where $B \equiv 1 /\{\alpha[1+b(\beta)]\}^{2 \beta-1}$. The redshift dependence is implicitly contained in the function $B$. If $\beta=2 / 3$, Eq. 2.125 coincides exactly with the core-halo mass relation in [4], i.e. Eq. 2.105).

\subsection{Conclusions and Discussions}

In this chapter, I introduced some basic knowledge and tools that are widely used in cosmology. The linear perturbation theory within the standard cold dark matter (CDM) model and the fuzzy dark matter (FDM) model is compared in detail. Unlike in the CDM model, where linear overdensities grow on all scales due to the gravitational instability, in FDM model the coherently oscillating scalar fields give rise to a scale-dependent sound speed that suppresses the growth of overdensity below the Jeans length. Thus the linear matter power spectrum of FDM is largely suppressed on small scales.

I then discussed the spherical collapse model. For CDM, analytic solutions can be obtained. Overdense regions will collapse to a halo once they exceed the critical value $\delta_{c} \approx 1.686$ which is independent of the size of the region. For FDM, due to the non-vanishing scale-dependent sound speed, or equivalently non-vanishing pressure (the so-called "quantum pressure"), the collapse happens later for the same initial overdensity. If the scale of the initial overdensity is smaller than the Jeans length, the gravity will be balanced by the quantum pressure, thus no collapse happens. Therefore, the critical overdensity for collapse is scale-dependent. With one-dimensional simulations, I confirm several features of FDM that have been discussed a lot in previous literature: (1) FDM halos collapse later; (2) FDM halos have flat cores. With more detailed analysis, the simulation can be extended to obtain the scale-dependent critical overdensity for collapse, which so far has only been estimated from the linear perturbation theory.

The results from the linear perturbation theory and the simplified spherical collapse model can be used as an input for the Press-Schechter (PS) formalism [89] to compute the abundance of collapsed objects. I introduced the original PS formalism and a more self-consistent deducing method, i.e. the extended Press-Schechter (EPS) formalism [99]. In the EPS formalism, the collapse of a halo is analogous to a random walk first upcrossing the critical density. The halos mass function can then be obtained by solving the excursion set problems. This concept can be extended to computing the conditional mass function, i.e. the probability finding one halo's progenitor in a certain mass interval (see Section 2.1.6). Having this conditional mass 
function, one can successively draw branching events backward to find the merger history of one halo, i.e. constructing a merger tree (Section 2.1.7).

I introduce some basic concepts of the semi-analytic model for galaxy formation. In this dissertation, I only concentrate on the dark matter part and leave the part related to baryonic physic to future work. I show how we can implement FDM into the publicly available semi-analytic code GALACTICUS. Corresponding work has been described in our published paper [1].

Using the FDM transfer function with a sharp small-scale cutoff caused by quantum pressure and a modified barrier function accounting for the mass dependent growth of FDM [55], we apply the EPS formalism to calculate the HMF for FDM.

Our HMFs show minor differences compared to those derived from the ShethTormen (ST) formalism with a redefined critical overdensity for collapse [55] by having a larger cutoff mass that changes less strongly with redshift (cf. Fig. 2.10). We also compare our HMFs to the fitting HMFs from simulations by [33]. Our HMFs are slightly more consistent with the one by [33] at $z=0$ compared to the ST HMF for FDM. Comparing the cumulative density of halos with the observed abundance of ultra-faint lensed galaxies in the Hubble Frontier Fields [3], we found a similar lower constraint on FDM mass as in [3] (see Fig. 2.14).

Using the Cole2000 algorithm [105] implemented in GALACTICUS, we build synthetic merger trees for FDM and validate them by comparing the HMF with the solution of the excursion set approach at different redshifts. We find that the HMFs match reasonably well at redshift $z<3$ (see Fig. 2.15), while deviations exist at higher redshifts. We demonstrate, however, that the vast majority of subhalos accreted by the host at redshift $z>3$ is already completely merged with the host at $z=0$ and thus does not have a significant effect on the SHMF at the present time.

To study the nonlinear evolution of FDM subhalos, we modify an existing model for satellite halos (the 'orbiting' implementation) in GALACTICUS [123] which includes the nonlinear effects of dynamical friction and tidal stripping (we ignore tidal heating in the absence of calibrations for FDM simulations). In order to account for possible effects from compact solitonic cores in FDM halos, we disable the tidal stripping when a satellite has only its core left and change the merging criteria of subhalos. We find that if FDM composes a significant fraction of the total DM density, the SHMF is suppressed on small scales. The larger the fraction or the smaller the mass, the stronger is the suppression (see Fig. 2.20). For pure FDM, the SHMF exhibits a maximum around $4 M_{c}$ (see the right panel of Fig. 2.20), where $M_{c}$ is the mass of the solitonic core for subhalos close to the mass cutoff, reflecting the fact that pure 
cores are assumed to be stable against tidal stripping. We also consider the impact of modifying the concentration parameter for FDM halos on to the SMHF and find only weak differences.

The parameters for dynamical friction and tidal stripping used in our work were obtained from CDM $N$-body simulations. A recalibration of these parameters will be possible as soon as sufficiently large FDM simulations become available. In addition, we only considered gravitational interactions between the subhalos and their host. Simulations of FDM [29, 4] show that FDM halos have a granular structure caused by wave interference that might make the dynamics of FDM subhalos behave differently from the collisionless case. In particular, stripping may be more efficient than expected from purely gravitational tidal effects.

Besides, by considering the merger history of dark matter halos in scenarios with FDM, we offer an alternative explanation for the core-halo mass relation observed in cosmological simulations [29, 4]. This model is described in our published paper [5]. As above, we provide evidence for our model using stochastic merger trees and show that the core-halo mass relation depends only on the mass loss fraction of cores during binary mergers, $M_{c} \propto M_{h}^{2 \beta-1}$. We find that for $\beta=0.7$ [62], this relation fits numerical data from cosmological simulations very well [4].

In addition to providing a simple explanation for the core-halo mass relation, we have shown that the core mass is determined by the individual accretion history. It can be modeled more realistically using individual mass accretion histories that recover not only the mean core-halo mass relation but also its scatter. 


\section{Chapter 3}

\section{Tidal Disruption of FDM Cores}

In the last chapter, using the semi-analytic code GALACTICUS modified for FDM, we have obtained the subhalo mass function (SHMF) for Milky Way-like FDM halos. One of the modification is to set the mass loss caused by tidal stripping to 0 once the halo has only its core left. It is motivated by the simple assumption that the compact solitonic core is stable against tidal perturbing. This assumption has led to a pronounced peak in the SHMF (see the right panel of Fig. 2.20) which may be a unique signature of FDM. However, the assumption itself has not been tested. This motivates us to do a detailed study of FDM cores under tidal stress.

In [6], tidal mass loss of a solitonic core orbiting inside a host halo is computed in a "tunneling approximation" by adding a spherical tidal potential to the timeindependent Schrödinger-Poisson (SP) equations. The mass loss rate is obtained from the imaginary part of the (complex) energy eigenvalue $E$. Since both tidal and gravitational potentials are taken to be time independent, so is the tidal radius. Consequently, the mass loss is fully characterized by the decreasing amplitude of the wave function.

As shown below, the actual dynamics are more complicated and can only be studied with numerical simulations. Once mass outside the tidal radius is removed, the core relaxes to a new ground state with smaller mass and accordingly larger core radius. In the process, mass is transferred through the tidal radius and subsequently stripped away resulting in a continuous loss.

This process has a classical analog. After the outer parts of the satellite are stripped away, the remnant is no longer in virial equilibrium and needs to convert kinetic to potential energy in order to re-equilibrate [151, 152]. The resulting configuration has a larger characteristic radius and decreased density, achieved by an outwards directed mass transfer through the tidal radius which, in turn, shrinks as 
a result of the lowered enclosed mass. However, for CDM, as shown in [152], this process is usually not sufficient to disrupt the subhalo.

\subsection{Classical and Tunneling Tidal Radius}

A satellite halo orbiting the host halo loses its mass due to the tidal force of the host halo, i.e. the tidal stripping effect (see also Section 2.3.4). Considering a satellite orbiting its host with synchronous rotation, i.e. the angular velocity of self-rotation equals the orbital angular velocity, the tidal radius can be calculated from classical Newtonian dynamics [146]:

$$
r_{t}=\left(\frac{G M_{\mathrm{sat}}\left(<r_{t}\right)}{\omega^{2}-d^{2} \Phi / d x^{2}}\right)^{1 / 3}
$$

where $M_{\text {sat }}$ is the satellite mass enclosed within the tidal radius, $\omega$ is the angular velocity of the satellite, $\Phi$ is the gravitational potential of the host halo, and $x$ is the distance to the host's center. Assuming a circular orbit of the satellite and most of the host mass to be within the orbital radius, we have

$$
\frac{d^{2} \Phi}{d x^{2}}=-\frac{2 G M_{\text {host }}\left(<x_{\mathrm{sat}}\right)}{x_{\mathrm{sat}}^{3}}=-2 \omega^{2} .
$$

Then the tidal radius can be written as

$$
r_{t}=\left(\frac{G M_{\mathrm{sat}}\left(<r_{t}\right)}{3 \omega^{2}}\right)^{1 / 3} .
$$

In [6], tidal stripping of FDM halos is treated quantum-mechanically by adding a spherical tidal potential to the Schrödinger equation. The authors propose that mass inside the tidal radius can be stripped in sufficiently long time due to tunneling.

Following this approach, we first consider a simple system in which the solitonic core is subject to a spherically symmetric tidal potential $\Phi_{t}=-\gamma \omega^{2} r^{2}$ (here, $r$ is the distance to the center of the satellite). Note that [6] use $\gamma=\frac{3}{2}$ which includes the effect of the centrifugal force owing to synchronous rotation of the satellite, assuming it to be a rigid body. However, a solitonic core forms an irrotational Riemann-S ellipsoid when subject to the tidal force as discussed in Section 3.4. Therefore, for a solitonic core, $\gamma$ in the tidal potential should be between 1 (without self-rotation) and $\frac{3}{2}$ (with uniform self-rotation that equals the orbital angular velocity). To be comparable with [6] we fix $\gamma$ to $\frac{3}{2}$ unless specified otherwise. 
Working in a coordinate system centered on the satellite, the SP equations become

$$
\begin{aligned}
i \hbar \frac{\partial \psi}{\partial t} & =-\frac{\hbar^{2}}{2 m_{a}} \nabla^{2} \psi+m_{a}\left(\Phi-\Phi_{t}\right) \psi \\
\nabla^{2} \Phi & =4 \pi G m_{a}|\psi|^{2}
\end{aligned}
$$

where $\Phi_{t}$, as defined previously, is an external potential accounting for the tidal force from the host. Assuming a spherical tidal force, $\Phi_{t}=-\frac{3}{2} \omega^{2} r^{2}$.

In [6], the authors decompose the wave function $\psi(r, t)=\phi(r) \exp (-i E t)$ to get the time-independent SP equations. Assuming the energy eigenvalue $E$ to be complex, they obtain the mass loss rate from the imaginary part of $E$,

$$
\frac{\dot{M}}{M}=\frac{\dot{\rho}}{\rho}=2 \operatorname{Im}(E),
$$

which only depends on the density ratio between the central density of the soliton $\rho_{c}$ and the average density of the host within the orbital radius $\bar{\rho}_{\text {host }}$, i.e. $\mu \equiv \rho_{c} / \bar{\rho}_{\text {host }}$. By solving the eigenvalue problem as in [6], we find a fitting formula for the imaginary part of $E$ :

$$
\operatorname{Im}(E)=-T_{\text {orbit }}^{-1} \exp \left[a\left(\frac{3}{2 \gamma} \mu\right)^{2}+b\left(\frac{3}{2 \gamma} \mu\right)+c\right],
$$

with the best-fitting parameters $\{a, b, c\}=\left\{5.89794 \times 10^{-5},-8.72733 \times 10^{-2}, 1.6774\right\}$. Here, $T_{\text {orbit }} \equiv 2 \pi / \omega$ is the orbital period. Fig. 3.1 shows out fitting formula Eq. (3.7) compared to the one obtained by solving eigenvalue problem as in [6].

However, as the gravitational potential depends on the amplitude of $\psi(r, t)$, in principle, we cannot separate $\psi(r, t)$ into two parts which purely depend on time and radius, respectively. Therefore, the treatment in [6] can only be seen as an approximation for small enough time scales on which the gravitational potential can be treated as time independent. As the solitonic core loses some of its mass and becomes less dense, it is increasingly vulnerable to tidal forces.

To test these arguments, we set up two special simulations using a pseudospectral solver (see the next section for details). First, we assume that the gravitational potential $\Phi$ in Eqs. (3.4) and (3.5) does not change with time. By solving the eigenvalue problem, we obtain the ground eigenstate with the parameter $\mu=50$ and use it as the initial condition. Then we solve the time-dependent Schrödinger equation [Eq. (3.4)] assuming that $\Phi$ does not change. Finally, for comparison we allow $\Phi$ to be time dependent and solve the full SP equations with the same initial condition. The results are shown in Fig. 3.2. As can be seen, if $\Phi$ does not change with time, the evolution of the core's central density exactly matches the prediction in [6]. But if we consider 


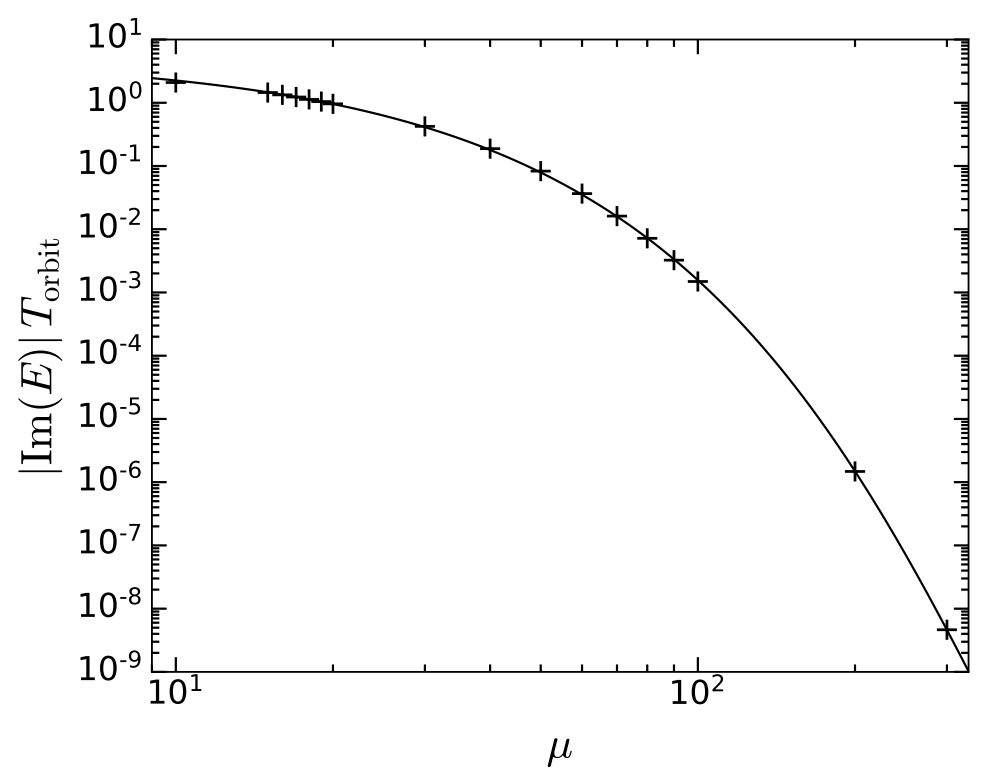

Figure 3.1: The imaginary part of the energy eigenvalue $E$. The solid line show the fitting formula Eq. (3.7). The crosses show the results from solving the eigenvalue problem as in [6].

the full nonlinear problem, the evolution of the central density is consistent with the prediction only at the very beginning, afterwards the central density decreases more quickly.

The density profile of solitonic cores can be approximated by

$$
\rho_{s}(r)=\frac{\rho_{c}}{\left[1+\left(\alpha r / r_{c}\right)^{2}\right]^{8}}
$$

where $\rho_{c}$ is the central density and $r_{c}$ is the radius where the density drops to half of the central density [29, 4, 31]. We will call $r_{c}$ the core radius and set $\alpha=0.302$ as in [29, 4] hereafter. As a result of the scaling relation $(r, \psi, \Phi, E, \omega) \rightarrow$ $\left(r / \lambda, \lambda^{2} \psi, \lambda^{2} \Phi, \lambda^{2} E, \lambda^{2} \omega\right)$, it follows that $\rho_{c} \sim r_{c}^{-4}$. Calculating the gravitational potential of a solitonic core from Eq. (3.8), the tidal radius can be obtained via Eq. (3.3). It is easy to check with the help of the scaling relation that the tidal radius in units of the core radius, $r_{t} / r_{c}$, only depends on the density ratio $\mu$.

Figure 3.3 shows regions inside (blank region) and outside (shaded region) the tidal radius with respect to the density ratio. For $\mu \gtrsim 30.4$, more than $95 \%$ of the total soliton mass is within the tidal radius. 


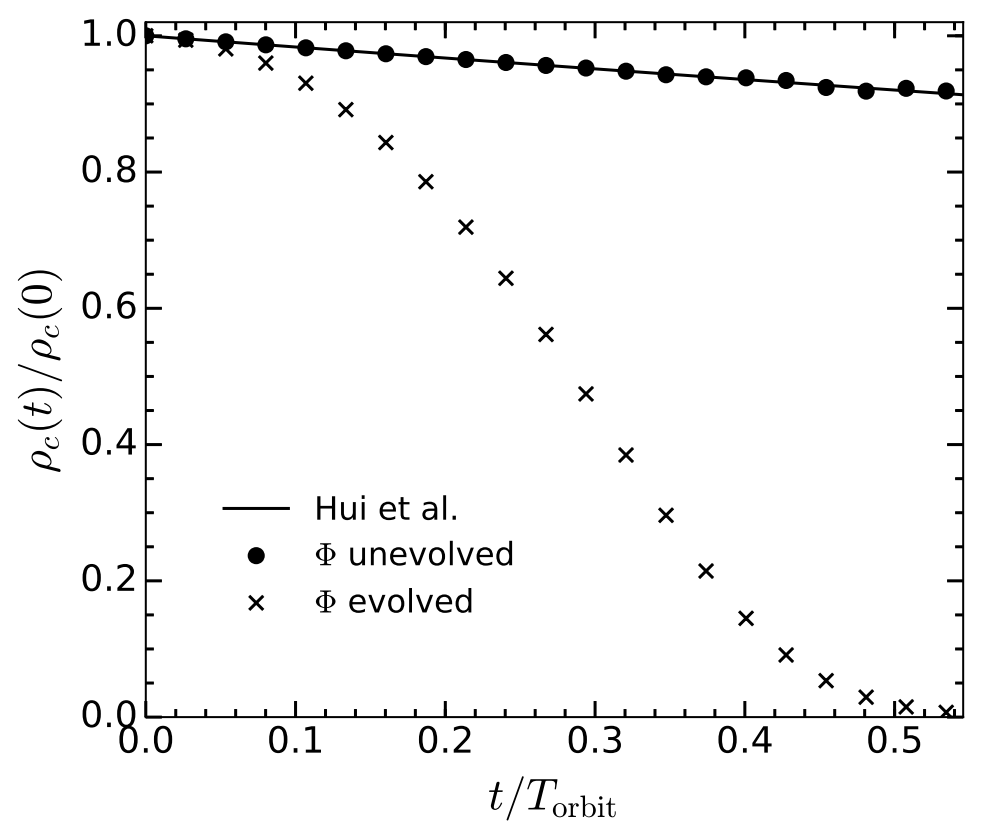

Figure 3.2: Evolution of the core's central density with unevolved (dots) and evolved (crosses) gravitational potential compared to the prediction in [6]. Reproduced from [7], Fig. 1.

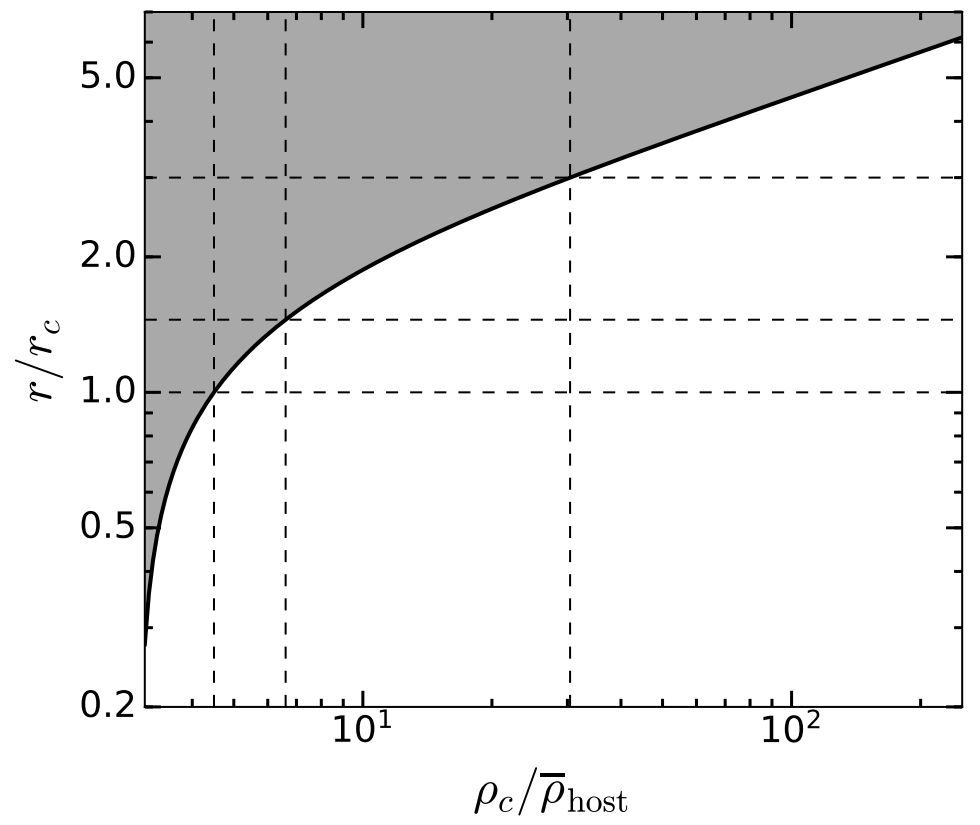

Figure 3.3: Regions inside (blank region) and outside (shaded region) the tidal radius with respect to the density ratio $\mu \equiv \rho_{c} / \bar{\rho}_{\text {host }}$. The solid line shows the tidal radius computed from Eq. (3.3). The horizontal lines mark the radii enclosing 95\% (top), $50 \%$ (middle) and 25\% (bottom) of the total soliton mass, respectively. When $\mu<4.5$, the tidal radius is smaller than the core radius. Thus, the solitonic core becomes unstable and is quickly disrupted. Reproduced from [7], Fig. 2. 


\subsection{Numerical Method}

\subsubsection{Pseudospectral Method}

To investigate fully time-dependent tidal stripping of solitonic subhalo cores with increasingly relaxed symmetry assumptions, we conducted numerical simulations of the spherically symmetric [Eqs. (3.4) and (3.5)] and the full three-dimensional problem.

In the spherically symmetric case, we use the ground state of Eqs. (3.4) and (3.5) as initial conditions and work with dimensionless quantities as in [150]. All the results presented here can be rescaled to restore the physical units. The boundary is set at $r_{\max }=280$ (as a reference, the initial core radius is at about $r_{c}=1.308$ ). We implement an absorbing boundary condition by adding a "sponge" (imaginary potential) in the outer regions of the grid,

$$
V(r)=-\frac{i}{2} V_{0}\left\{2+\tanh \left[\left(r-r_{s}\right) / \delta\right]-\tanh \left(r_{s} / \delta\right)\right\} \Theta\left(r-r_{p}\right),
$$

where $\Theta$ is the Heaviside function [150]. We set $r_{p}=2 / 7 r_{\max }, r_{s}=\left(r_{\max }+r_{p}\right) / 2$, $\delta=\left(r_{\max }-r_{p}\right)$, and $V_{0}=2$.

In the full three-dimensional case, we work in coordinates centered on the host. Instead of adding a tidal potential $\Phi_{t}$ to the Schrödinger-Poisson (SP) equations, we add a static host gravitational potential and let the soliton orbit around the host. The host is treated as a uniform sphere with mass $M_{\text {host }}$ and a radius of roughly 10 times the cell size. Contrary to the spherically symmetric case, no eigenstate is available. We therefore use soliton solutions as initial conditions and assume periodic boundary conditions. The soliton is placed initially at a distance of $D=25$ to the host and is given an initial velocity $v_{0}=\left(G M_{\text {host }} / D\right)^{1 / 2}$. The simulated box has a length of 160 on each side and totally $480^{3}$ cells so that the core radius is covered by at least 4 cells. We have verified that the artificial sponge is not necessary in this case over the entire simulation time.

To solve the SP equations, we have developed a fourth-order pseudospectral solver. It provides fourth-order convergence in time and spectral convergence in space. Compared to previous second-order pseudospectral methods, e.g. [27, 124, our code is about 6 times faster in getting comparable accuracy.

The wave function is advanced in time by a unitary transformation,

$$
\psi(t+\Delta t)=\exp (-i H \Delta t) \psi(t)
$$

where $H$ is the Hamiltonian of the system which can be split into the kinetic part $K$ and the potential part $W$, i.e. $H=K+W$. In general, the operator $\exp (-i H \Delta t)$ 
can be expanded as

$$
\exp (-i H \Delta t)=\prod_{i} \exp \left(-i t_{i} K \Delta t\right) \exp \left(-i v_{i} W \Delta t\right)
$$

where $t_{i}$ and $v_{i}$ are parameters to be determined by the requirements of the chosen order. For example, to second order we obtain the well-known leapfrog method,

$$
\exp (-i H \Delta t)=e^{-\frac{i}{2} W \Delta t} e^{-i K \Delta t} e^{-\frac{i}{2} W \Delta t}+O\left(\Delta t^{3}\right)
$$

which is also referred to as the "kick-drift-kick" formulation. If we exchange the operators $K$ and $W$ in Eq. (3.12), i.e. update the position first, we arrive at the "drift-kick-drift" formulation which also has second-order accuracy.

In our simulations, we implement the fourth-order algorithm proposed by McLachlan [153],

$$
\begin{aligned}
e^{-i H \Delta t} \approx \quad & e^{-i v_{2} W \Delta t} e^{-i t_{2} K \Delta t} e^{-i v_{1} W \Delta t} e^{-i t_{1} K \Delta t} e^{-i v_{0} W \Delta t} \\
& e^{-i t_{1} K \Delta t} e^{-i v_{1} W \Delta t} e^{-i t_{2} K \Delta t} e^{-i v_{2} W \Delta t}
\end{aligned}
$$

where

$$
\begin{aligned}
& v_{1}=\frac{121}{3924}(12-\sqrt{471}), \quad w=\sqrt{3-12 v_{1}+9 v_{1}^{2}}, \\
& t_{2}=\frac{1}{4}\left(1-\sqrt{\frac{9 v_{1}-4+2 w}{3 v_{1}}}\right), \quad t_{1}=\frac{1}{2}-t_{2}, \\
& v_{2}=\frac{1}{6}-4 v_{1} t_{1}^{2}, \quad v_{0}=1-2\left(v_{1}+v_{2}\right) .
\end{aligned}
$$

Compared to the leapfrog method, it is much more accurate. Note that the kinetic operator is performed in Fourier space, while the potential operator is performed in real space. In Fourier space the kinetic operator can be computed in a very simple way: $e^{-i K} \hat{\psi}=e^{-i \hbar^{2} k^{2} / 2 / m_{a}} \hat{\psi}$. The potential $W$ is obtained by solving the Poisson equation via a spectral method [27, 124].

\subsubsection{Convergence Tests}

To test the convergence of our code in time, we simulate mergers of multiple solitons, which have been studied in detail in previous papers, e.g. [4, 62, 124]. The simulated box has a length of 40 on each side and a resolution of $240^{3}$ cells. At the initial time, 20 solitons with the same core radius $r_{c}=1.308$ are randomly put in the box. We check the conservation of the total energy and compare it with the well-known second-order algorithm, kick-drift-kick leapfrog method. 


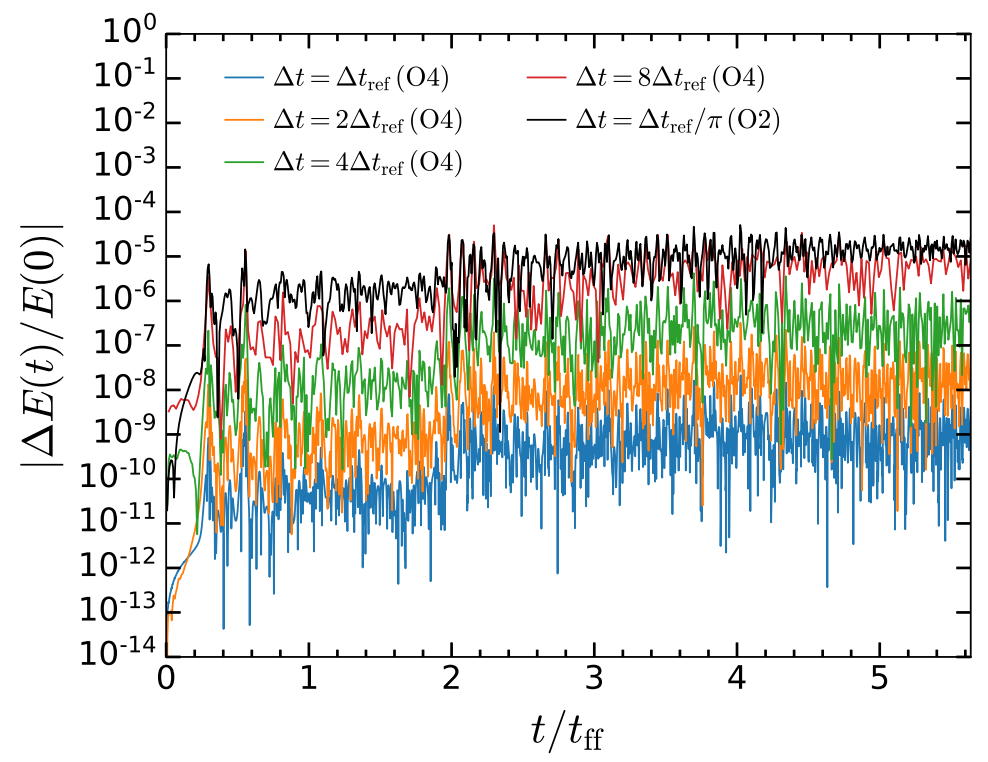

Figure 3.4: Numerical error of the total energy with respect to time for different time step sizes and different algorithms. The time is in units of the free-fall time scale $t_{\mathrm{ff}}=\sqrt{3 \pi /(32 G \rho)}$ with $\rho$ equal to the average density over the whole simulated box. Here "O4" refers to the fourth-order algorithm we used in our simulations (Eq. 3.13). "O2" refers to the second-order kick-drift-kick formulation Eq. (3.12), which is widely used in previous simulations. Reproduced from [7], Fig. 11.

Figure 3.4 shows the relative error of the total energy with respect to time for the fourth-order algorithm (O4, colored lines) and the second-order algorithm (O2, black line). Results from simulations with different time step sizes are shown. As can be seen, the fourth-order algorithm has comparable accuracy to the second-order algorithm even if the time step size is $8 \pi$ times larger.

Figure 3.5 shows the average relative error of the total energy with respect to the time step size. As expected the algorithm we implemented (circles) has fourth-order convergence. Compared to the second-order algorithm (squares), it converges faster and the error is several orders of magnitude smaller if the same time step size is used.

\subsection{Core Mass Loss Rate from Simulation}

\subsubsection{Spherically Symmetric Approximation}

We simulate the evolution of cores with different initial density ratios $\mu$. The mass within the tidal radius $M_{t}$ is computed at different times. The mass loss rate is then 


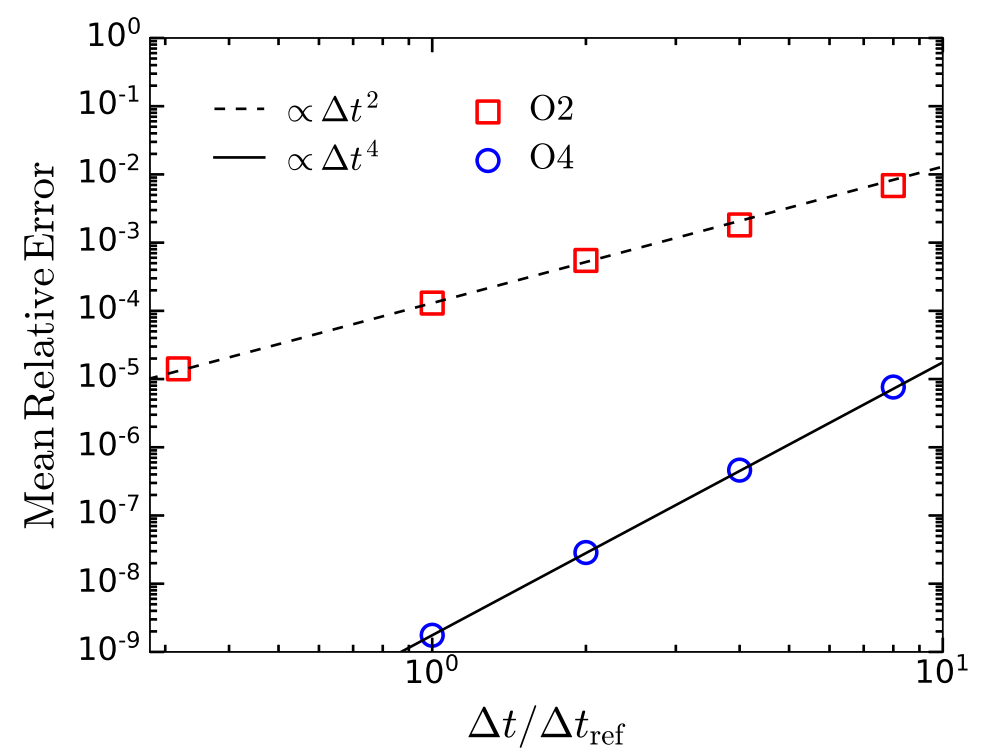

Figure 3.5: Average numerical error of the total energy with respect to the time step size. Only data with $t>3 t_{\mathrm{ff}}$ when the numerical error oscillates around roughly a constant value is included in the analysis. Reproduced from [7, Fig. 12.

calculated by

$$
\begin{aligned}
\frac{\dot{M}_{t}}{M_{t}} & =\frac{1}{M_{t}} \frac{d}{d t} \int_{0}^{r_{t}(t)} 4 \pi r^{2} \rho(r, t) d r \\
& =\frac{1}{M_{t}} \int_{0}^{r_{t}(t)} 4 \pi r^{2} \partial_{t} \rho(r, t) d r+\frac{1}{M_{t}} 4 \pi r_{t}^{2} \rho\left(r_{t}, t\right) \dot{r}_{t},
\end{aligned}
$$

where the first term can be interpreted as mass transfer through the tidal radius and the second term corresponds to effects of a decreasing tidal radius. Using Gauss' theorem we can rewrite the first term as

$$
\frac{1}{M_{t}} \int_{0}^{r_{t}(t)} 4 \pi r^{2} \partial_{t} \rho(r, t) d r=-\frac{1}{M_{t}} \oiint_{r=r_{t}} \rho(r, t) \mathbf{v}(r, t) \cdot d \mathbf{S},
$$

where $\mathbf{v}$ is the velocity field.

Figure 3.6 shows both contributions to the mass loss rate. For larger density ratios $\mu$, the first term in Eq. 3.15) (dashed colored lines) dominates. With decreasing $\mu$, the second term (shaded region) becomes more important. The mass loss rate due to mass transfer through the tidal radius is close to the prediction in [6] which they attribute to tunneling effects, as long as we take the decreasing of the core's central density into account. It is only about $30 \%$ larger than the prediction. I

\footnotetext{
${ }^{1}$ The results were confirmed by solving the Poisson equation using a Numerov Algorithm, while the wave function was evolved with a fully implicit Crank-Nicholson scheme. The same imaginary potential Eq. (3.9) was used.
} 


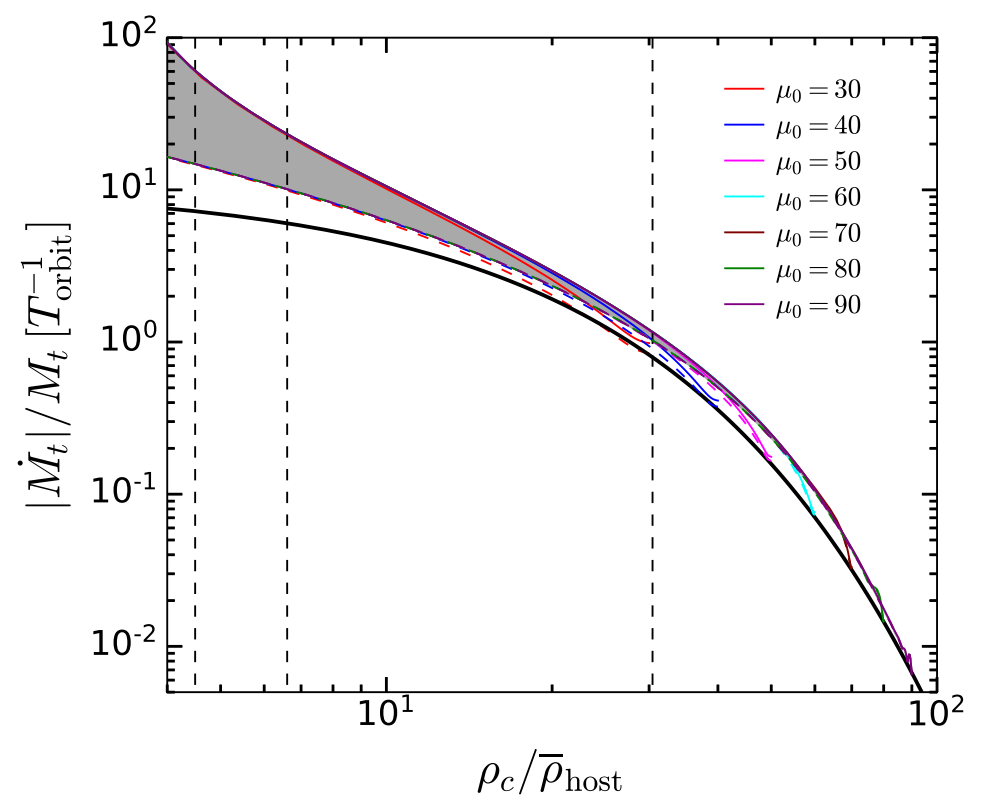

Figure 3.6: Mass loss rate for different initial density ratios. A spherical tidal field is assumed. The solid black line shows the prediction in [6] using the fitting formula Eq. (3.7). The vertical dashed lines are the same as in Fig. 3.3. The solid lines with different colors give the total mass loss rate. The dashed colored lines show the mass loss rate by mass transfer through the tidal radius, while the shaded region corresponds to the mass loss rate from the decreasing tidal radius. Reproduced from [7], Fig. 3. 


\subsubsection{Three-dimensional Simulation}

We now consider the three-dimensional case (full model). There are two major differences between the simplified model and the full model: (1) in the former, the subhalo is assumed to be in a state of synchronous rotation, i.e. the subhalo rotates like a rigid body $(\nabla \times \mathbf{v} \neq 0)$, which cannot be true for FDM cores whose velocity field is curl-free; (2) the simplified model assumes a spherically symmetric tidal force, so the solitonic core spins at a constant rate. On the contrary, in the full model, the solitonic core can spin up due to tidal torque (see Sec. 3.4 for detail).

In the three-dimensional case, it is difficult to find a well-defined tidal radius. Therefore, instead of analyzing the mass within the tidal radius, we will look at the evolution of the core mass $M_{c}$, defined as the enclosed mass within the core radius $r_{c}$ (about $1 / 4$ of the total soliton mass). In our simulations, we find that after the core loses some of its mass, it quickly relaxes to a new soliton profile with a smaller central density (see Fig. 3.7). From the density profile of solitons, Eq. (3.8), we can see that the core mass $M_{c} \propto \rho_{c}^{1 / 4}$. Therefore we need to adjust Eq. (3.6) accordingly:

$$
\frac{\dot{M}_{c}}{M_{c}}=\frac{1}{4} \frac{\dot{\rho}_{c}}{\rho_{c}}=\frac{1}{2} \operatorname{Im}(E) .
$$

Figure 3.8 presents slices through the density field at different times for $\mu=50$. The thick and thin contour lines mark where the density drops to $50 \%$ (core radius) and $1 \%$ of the maximum density, respectively. For comparison, we also show the tidal radius computed from the spherically symmetric approximation (dashed circles). As can be seen, the core loses mass gradually but since the gravitational time scale is smaller than the mass loss time scale, it quickly relaxes to a new soliton state with a lower central density (upper-right plot, see also Fig. 3.7). At $t=4.36 T_{\text {orbit }}$ (lower-left plot), the tidal radius is comparable to the core radius. Afterwards, in less than one orbit, the core is totally disrupted and leaves only a long tail behind (lower-right plot).

Figure 3.9 illustrates the core mass loss rate from simulations with different initial conditions compared to the prediction from Eqs. (3.7) and (3.17). In general, the results are close to the predictions. At very early times, the core mass decreases more slowly than the prediction. This can be attributed to the initial conditions. We initially assume a soliton without self-rotation, i.e. the proportionality coefficient in the tidal potential should be $\gamma=1$ at the beginning. The core subsequently acquires angular momentum and starts to spin up due to tidal torque, so $\gamma$ approaches $3 / 2$. For comparison, the dotted line shows the prediction with $\gamma=1$. As can be seen, at 


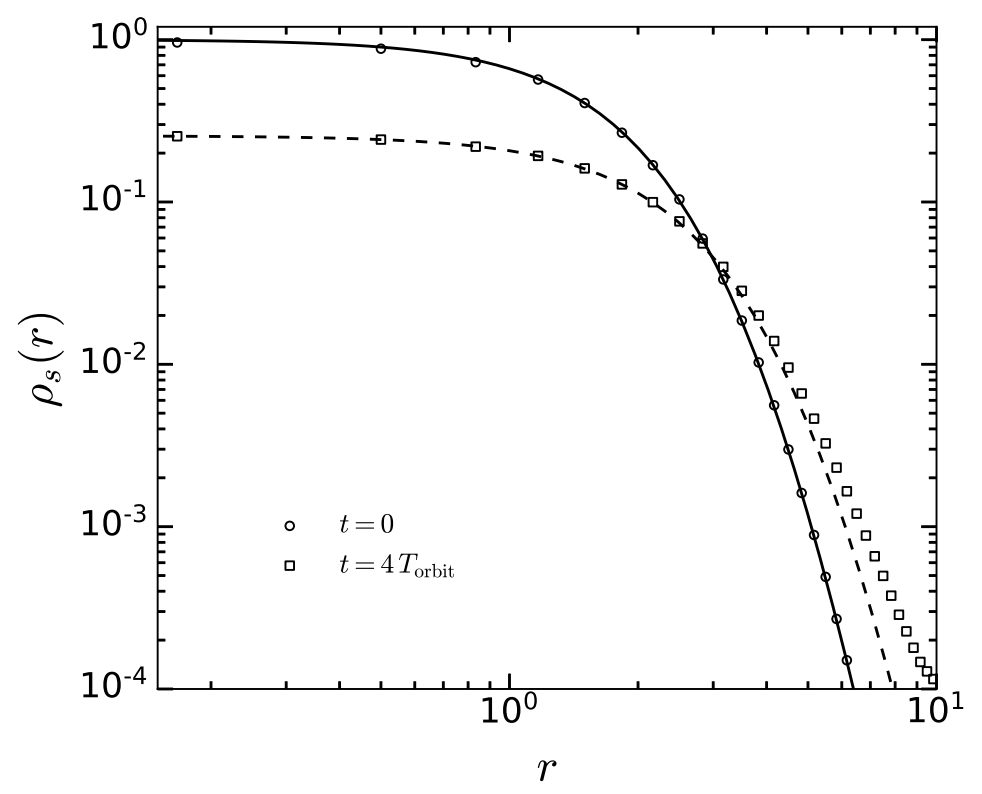

Figure 3.7: The density profile of the core at the initial time and at $t=4 T_{\text {orbit }}$ for a density ratio $\mu=50$. The circles and squares show the average radial density profile obtained from the simulation. The lines display fitted profiles defined in Eq. (3.8). It can be seen that the cores are well described by soliton profiles even after losing substantial amounts of mass. Reproduced from [7], Fig. 4.

early times the mass loss rate computed from simulations roughly falls between the solid and dotted curves.

\subsection{Tidal Locking}

One important difference between FDM subhalo cores and rigid-body satellites is that the solitonic core does not sustain uniform self-rotation. In our simulations, we find that an initially spherical solitonic core without self-rotation gradually spins up and forms an irrotational ellipsoid in the tidal field of the host. The velocity field in a typical simulation with $\mu=50$ can be seen in Fig. 3.10. Inside the tidal radius (dashed circle, computed from the spherically symmetric approximation), the velocity field is characteristic for an irrotational Riemann-S ellipsoid [8]. The core is elongated towards the host's center (the hollow arrow in Fig. 3.10), indicating that the core is tidally locked. However, unlike a rigid body, the core does not rotate uniformly.

As the central density of the core decreases over time, the shape of the ellipsoid also changes. Denote the semiaxes of the core as $a_{1}, a_{2}$, and $a_{3}\left(a_{1} \geq a_{2} \geq a_{3}\right)$. Then the change can be characterized by the eccentricities of the ellipsoid. In the middle panel of Fig. 3.11, we show the evolution of the eccentricity of the ellipsoid in the 


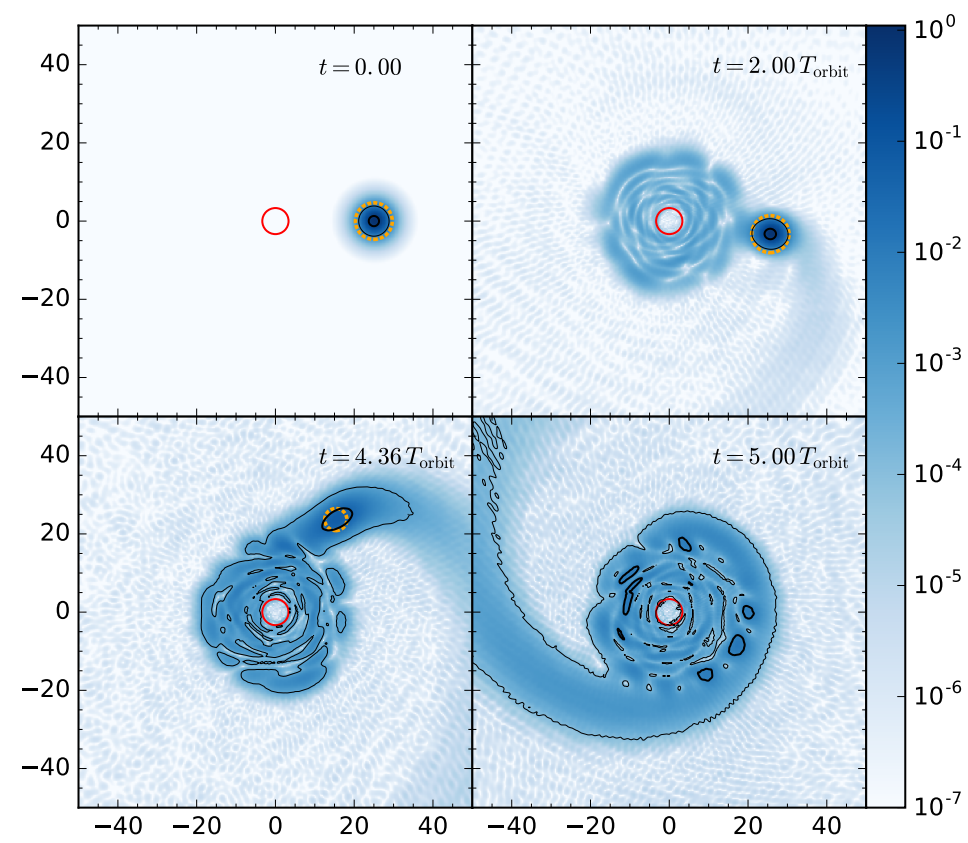

Figure 3.8: Slices through the density field at different times for an initial density ratio $\mu=50$. The circle in the center of each plot indicates the size of the host (for simplicity the host is treated as a small sphere with uniform density). The thick and thin contour lines mark where the density drops to $50 \%$ (core radius) and $1 \%$ of the maximum density, respectively. The dashed circles show the tidal radii computed from the spherically symmetric approximation. Reproduced from [7], Fig. 5.

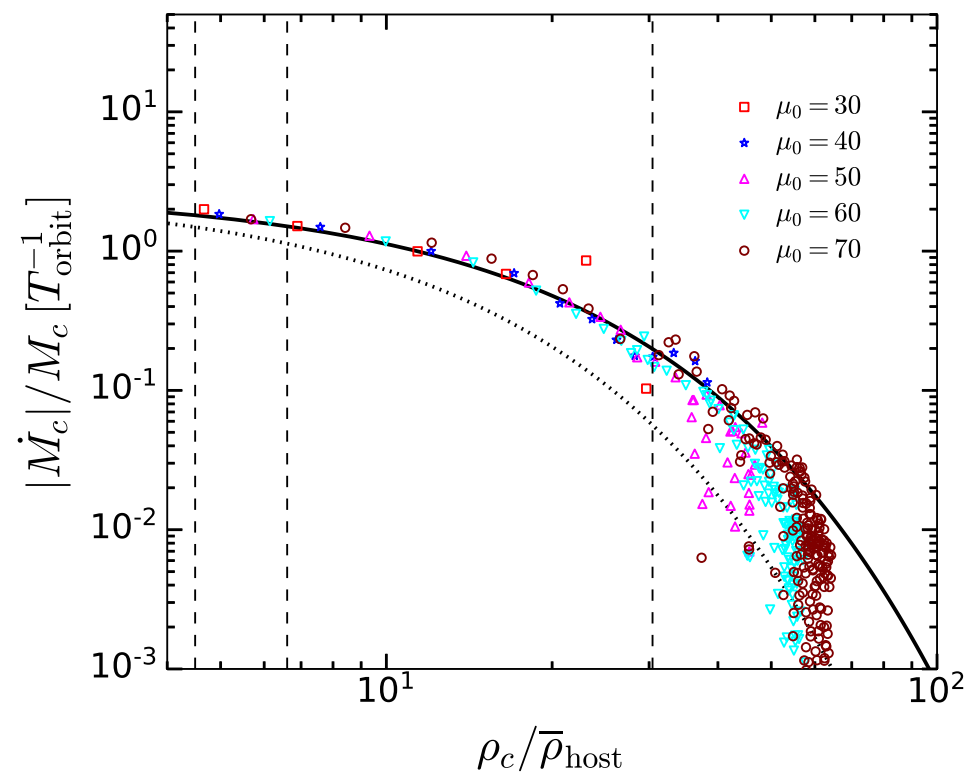

Figure 3.9: Core mass loss rate for different initial density ratios. The lines show the prediction from Eq. (3.7) and Eq. (3.17) with $\gamma=3 / 2$ (solid line) and $\gamma=1$ (dashed line). The vertical dashed lines are the same as in Fig. 3.3. Reproduced from [7], Fig. 6 . 


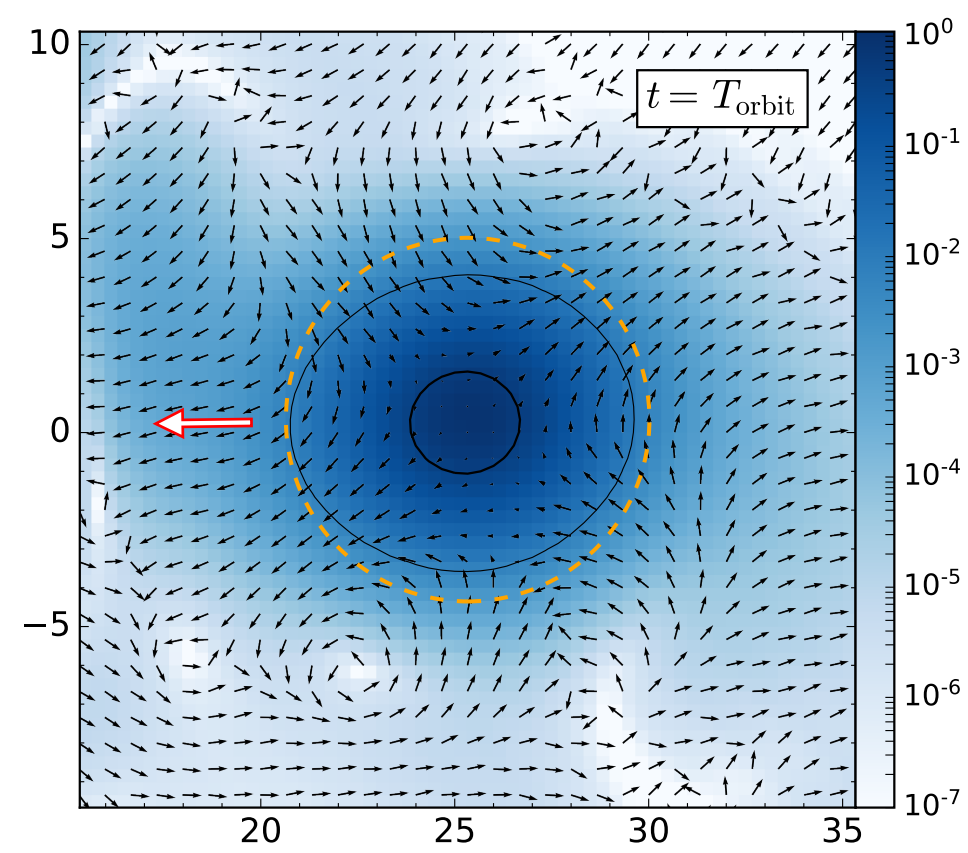

Figure 3.10: Slice through the core. The color map indicates the density while the black arrows trace the velocity field relative to the core's collective motion. Inside the tidal radius (dashed circle) the velocity field is characteristic for an irrotational Riemann-S ellipsoid [8]. Outside the core, vortices can be seen. The thick black circle in the middle represents the core radius while the thin black ellipsoid marks the area where the density drops to one percent of the central density, i.e. almost all the mass lies within the tidal radius. The hollow arrow points towards the host's center. Reproduced from [7], Fig. 7. 
plane that contains the shortest and longest principal axes, i.e. $\epsilon_{13}=\left[1-\left(a_{3} / a_{1}\right)^{2}\right]^{1 / 2}$. We find that the eccentricity $\epsilon_{12}=\left[1-\left(a_{2} / a_{1}\right)^{2}\right]^{1 / 2}$ is very close to $\epsilon_{13}$, implying that the core is approximately spheroidal.

Assuming constant densities $\rho_{h}$ and $\rho_{\text {sat }}$ for the host and satellite, Roche found that the ellipticity of an equilibrated, tidally locked, fluid satellite can be calculated analytically as a function of its density ratio [154]

$$
\rho_{h} / \rho_{\mathrm{sat}}=\frac{1-\epsilon^{2}}{2 \epsilon^{3}}\left[\left(3-\epsilon^{2}\right) \operatorname{artanh} \epsilon-3 \epsilon\right] .
$$

Since the satellite's density inside the core radius does not change significantly, we set $\rho_{h} / \rho_{\text {sat }}=\mu$ and calculate the expected ellipticity from Eq. (3.18). The center panel of Fig. 3.11 confirms that this approximate solution agrees well with our numerical results as long as the core stays tidally locked.

The rotation of the core can be parameterized by the dimensionless spin parameter as defined in 155 for DM halos

$$
\lambda^{\prime}=\frac{L_{c}}{\sqrt{2} M_{c} V R},
$$

where $L_{c}$ is the core angular momentum with respect to its center, $R \equiv\left(a_{1} a_{2} a_{3}\right)^{1 / 3}$ is the mean core radius, and $V$ is the circular velocity at $R$. We show the evolution of $\lambda^{\prime}$ in the lower panel of Fig. 3.11 .

The top panel of Fig. 3.11 displays the angle between the longest principal axis of the core and $x$-axis, compared to the angle between the line joining the center of the core and the $x$-axis. It can be seen that the core becomes tidally locked in less than $1 / 4 T_{\text {orbit }}$.

From the center panel of Fig. 3.11, we see that the core eccentricity increases over time. In order for the core to stay tidally locked, the spin parameter has to increase as well. Thus the core will slightly deviate from tidal locking until it obtains additional angular momentum due to tidal torque and becomes tidally locked again. At late time, angular momentum transfer from orbital motion to self-rotation of the satellite becomes insufficient to maintain tidal locking. The core rotation lags behind its orbital frequency and the core quickly becomes tidally disrupted.

\subsection{Satellite Galaxies in the Milky Way}

Having quantified the core mass loss rate solely depending on the ratio $\mu$ between the central density $\rho_{c}$ of the satellite's core and the average host density $\bar{\rho}_{\text {host }}$, it is 


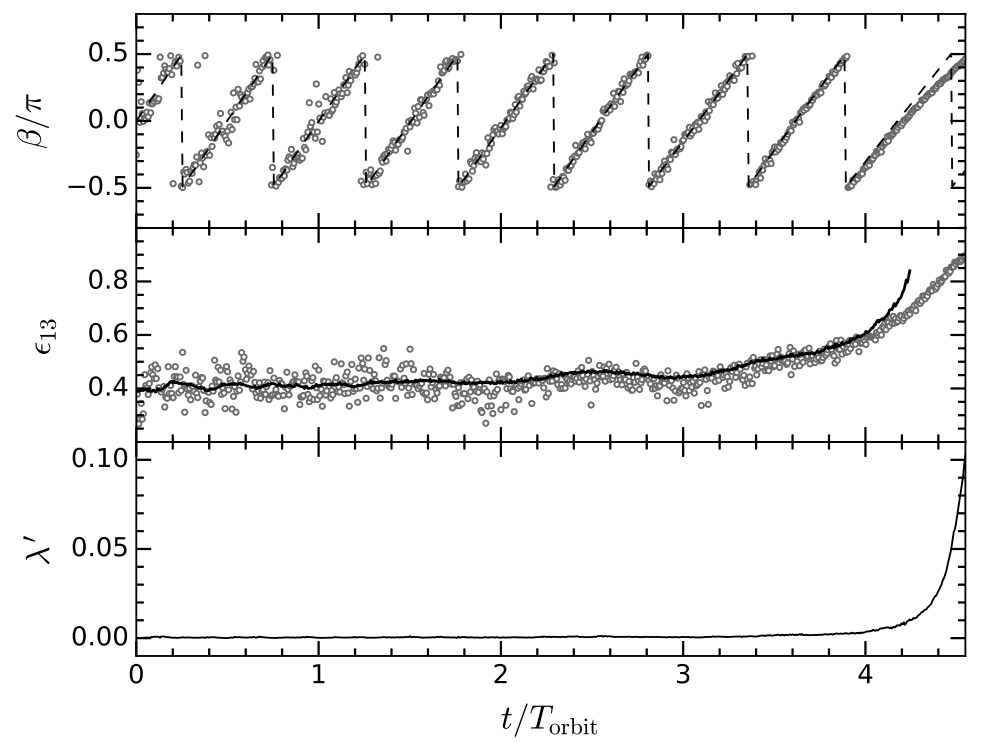

Figure 3.11: Representative run with initial density ratio $\mu=50$. Top: angle between the longest principal axis of the core and $x$-axis (grey circles). The dashed line shows the angle between the line joining the center of the core and the center of host and $x$-axis. It can be seen that the core is tidally locked most of the time. Center: eccentricity $\epsilon_{13}$ of the core. The solid line shows the expected values from Eq. (3.18). Bottom: spin parameter of the core. Reproduced from [7], Fig. 8.

straightforward to estimate the survival time of satellite galaxies in the Milky Way. Assuming a host mass of $M_{\text {host }}$, a given satellite distance $D$ to the galactic center directly translates into a mean host density $\bar{\rho}_{\text {host }}$ within the satellite's orbit. If we further assume that the satellite's core evolves along the fitting curve in Fig. 3.9, we can compute the minimum central density of the satellite required to survive for $N_{\text {sur }}$ orbits:

$$
\rho_{c, \min }=\mu_{\min }\left(N_{\text {sur }}\right) \bar{\rho}_{\text {host }}=\mu_{\min }\left(N_{\text {sur }}\right) \frac{3 M_{\text {host }}}{4 \pi D^{3}},
$$

where $\mu_{\min }\left(N_{\text {sur }}\right)$ is the minimum density ratio required. Furthermore, for a fixed FDM particle mass $m_{22} \equiv m /\left(10^{-22} \mathrm{eV}\right), \rho_{c}$ determines the core mass $M_{c} \propto \rho_{c}^{1 / 4}[29]$. Thus, the mass of the core surviving for $N_{\text {sur }}$ orbits must satisfy

$$
\begin{gathered}
M_{c}>5.82 \times 10^{8}\left[\mu_{\min }\left(N_{\mathrm{sur}}\right)\right]^{1 / 4} m_{22}^{-3 / 2}\left(\frac{D}{\mathrm{kpc}}\right)^{-3 / 4} \\
\left(\frac{M_{\mathrm{host}}}{10^{12} M_{\odot}}\right)^{1 / 4} M_{\odot} .
\end{gathered}
$$

If we consider the satellite to be disrupted when its core loses $90 \%$ of its mass and take $\gamma=3 / 2$ and $N_{\text {sur }}=10$, we find $\mu_{\min }=74$ which is slightly larger than estimated in [6]. Taking $\gamma=1$ and $N_{\text {sur }}=1$, we get a more conservative constraint $\mu_{\min }=8.4$. 
In Fig. 3.12, we use Eq. 3.21) for different FDM particle masses to constrain the minimum mass of cores that can survive for $N_{\text {sur }}$ orbits as a function of the distance to the Galactic center. The corresponding satellite mass should be larger than its core mass. We consider a Milky Way-like host $M_{\text {host }}=10^{12} M_{\odot}$. For comparison, we also show the half-light mass $M_{1 / 2}$ of some satellite galaxies in the Milky Way [9]. As expected, satellites close to the Galactic center are particularly susceptible to tidal disruption and therefore place the most stringent constrains on the particle mass. Specifically, the lightest satellites close to the Galactic center will only survive for more than one orbital time if the particle is as heavy as $m \simeq 2 \times 10^{-21} \mathrm{eV}$.

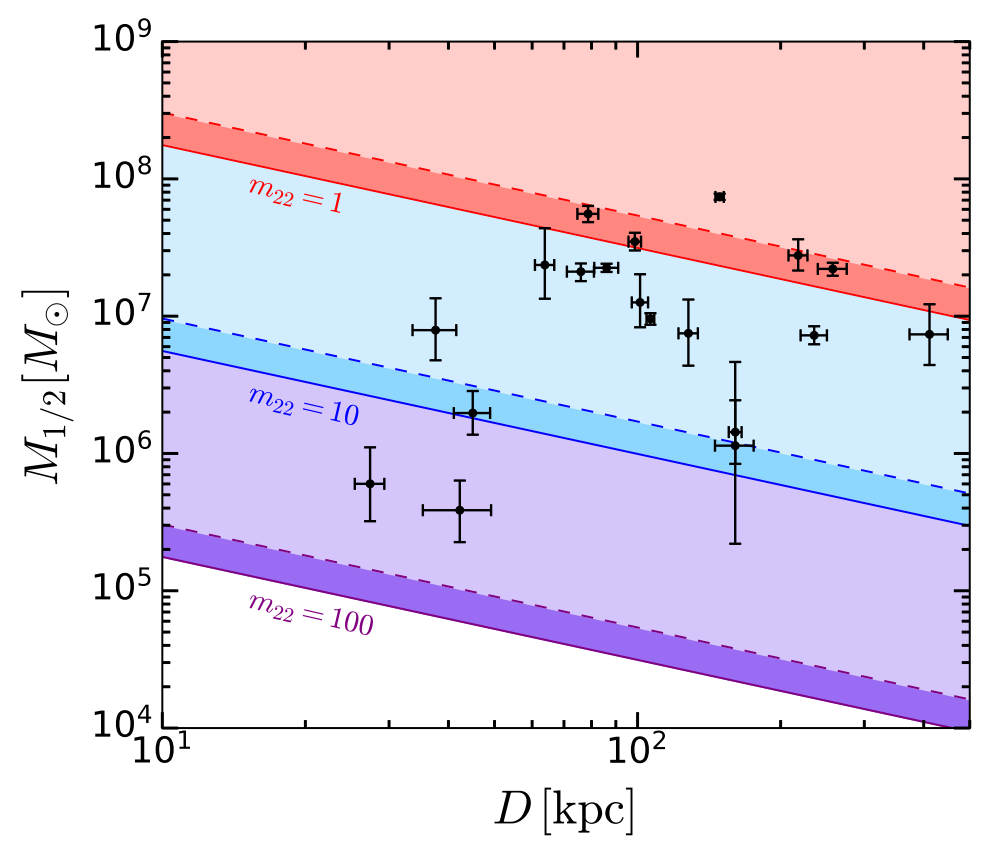

Figure 3.12: Minimum mass of cores that can survive for $N_{\text {sur }}$ orbits assuming different FDM particle masses $m_{22} \equiv m /\left(10^{-22} \mathrm{eV}\right)$ versus the distance to the Galactic center $D$. For comparison, we also show the half-light mass $M_{1 / 2}$ of some satellite galaxies in the Milky Way [9]. The mass of the host is taken to be $10^{12} M_{\odot}$. For each particle mass, the solid curve is obtained by assuming $\gamma=1$ and $N_{\text {sur }}=1$ while the dashed curve is obtained by assuming $\gamma=3 / 2$ and $N_{\text {sur }}=10$. To survive for $N_{\text {sur }}$ orbits, the half-light mass of satellite galaxies should be above the lines (assuming core mass is at most equal to the half-light mass). Reproduced from [7], Fig. 9.

\subsection{Effects on Subhalo Mass Function}

To study how tidal stripping of subhalos cores affects the SHMF, again we use GALACTICUS with modifications described in the last chapter. But additionally, we 
allow the core to lose mass due to tidal stripping. The core mass loss rate is computed from Eqs. (3.7) and (3.17) with $\gamma=3 / 2$ which is a good approximation to our numerical results (see Fig. 3.9). We note that a more recent version of the GALACTICUS code, v0.9.4 (revision a184070149c5), is used in the following computation. A bug in computing the tidal stripping mass loss rate is fixed in this revision. We find that the resulting SHMF for CDM is slightly more consistent with the results from N-body simulations at lower mass end even without recalibration 2 .

We run merger trees with 1000 trees per decade in mass for host halos with masses in the range $\left[1 \times 10^{12}, 3 \times 10^{12}\right] M_{\odot}$. The FDM mass ranges from $10^{-22} \mathrm{eV}$ to $10^{-21} \mathrm{eV}$. To resolve the smallest subhalos, we set the mass resolution of merger trees $M_{\text {res }}$ below the mass cutoff of the halo mass function. In general, we set $M_{r e s}=10^{7} M_{\odot}$. Note that $M_{\text {res }}$ is the resolution of merger trees, but subhalos are tracked till the merging criteria are satisfied (see Section 2.3.4). We also run merger trees for a higher FDM mass $m_{a}=5 \times 10^{-22} \mathrm{eV}$ with 100 trees per decade in mass. In this case, we set $M_{\text {res }}=10^{6} M_{\odot}$ since the cutoff of the halo mass function is lower for this FDM mass. For comparison, we run simulations with and without tidal stripping of subhalo cores. Finally, we also run merger trees for the standard CDM model with mass resolution $M_{\text {res }}=10^{7} M_{\odot}$.

The left (right) panel of Fig. 3.13 shows the SHMF for different FDM masses with (without) including tidal stripping of subhalo cores. As can be seen, if we assume the cores are stable against tidal stripping, the SHMF exhibits two peaks (as we showed in the last chapter). But if the the tidal stripping of cores is included, the first peak at lower masses is smeared out while the SHMF at higher masses is not affected.

We find that in both cases, with and without tidal stripping of subhalo cores, the FDM SHMF can be fitted by a universal formula

$$
\left.\frac{d N}{d \ln M}\right|_{\mathrm{FDM}}=f_{1}(M)+\left.\frac{d N}{d \ln M}\right|_{\mathrm{CDM}} f_{2}(M),
$$

with

$$
\begin{aligned}
& f_{1}(M)=\beta \exp \left[-\left(\ln \frac{M}{M_{1} \times 10^{8} M_{\odot}}\right)^{2} / \sigma\right] \\
& f_{2}(M)=\left[1+\left(\frac{M}{M_{2} \times 10^{8} M_{\odot}}\right)^{-\alpha_{1}}\right]^{-10 / \alpha_{1}} .
\end{aligned}
$$

\footnotetext{
${ }^{2}$ However, a recalibration may be needed to ensure that other properties of subhalos are also consistent with simulations.
} 

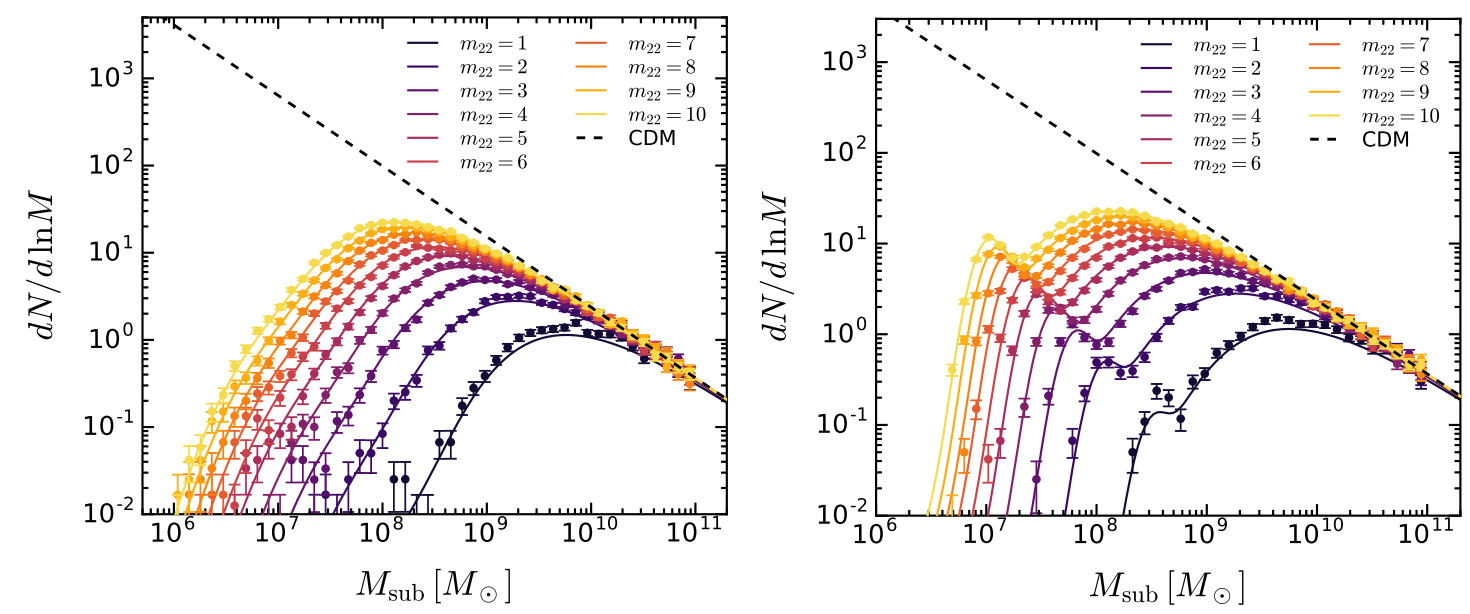

Figure 3.13: SHMF for different FDM masses. The solid and dashed curves are from the fitting functions Eqs. (3.22) and (3.25). Left: with tidal stripping of subhalo cores. Right: without tidal stripping of subhalo cores.

Here the function $f_{1}(M)$ accounts for the possible bumps in the SHMF at lower masses (see the right panel of Fig. 3.13). $f_{2}(M)$ characterizes the suppression in the SHMF for FDM compared to CDM, while the SHMF for CDM can be well fitted by

$$
\left.\frac{d N}{d \ln M}\right|_{\mathrm{CDM}}=a_{0}\left(\frac{M}{10^{8} M_{\odot}}\right)^{-\alpha_{0}},
$$

with $a_{0}=113.094$ and $\alpha_{0}=0.85858$. The fitting formula for CDM derived from GALACTICUS is very close to that obtained from N-body simulations by [156]. The best-fit values for parameters $\left\{\alpha_{1}, M_{1}, M_{2}, \beta, \sigma\right\}$ can be found in Table 3.1 .

\begin{tabular}{|c|c|c|}
\hline & Without stripping of cores & With stripping of cores \\
\hline$\alpha_{1}$ & 0.709259 & 0.719117 \\
\hline$M_{1} / m_{22}^{-3 / 2}$ & 3.35981 & 4.67990 \\
\hline$M_{2} / m_{22}^{-1.6}$ & 1.82263 & 1.97020 \\
\hline$\beta / m_{22}^{3 / 2}$ & 0.100882 & 0.0144670 \\
\hline$\sigma$ & 0.168529 & 1.41892 \\
\hline
\end{tabular}

Table 3.1: Best-fit parameters in the fitting SHMF for FDM. Here $m_{2} 2 \equiv$ $m_{a} /\left(10^{-22} \mathrm{eV}\right)$.

Note that to test the robustness of the fitting formula Eq. (3.22) under variation of the FDM mass, we perform the fitting to the cases with $m_{22} \equiv m_{a} /\left(10^{-22} \mathrm{eV}\right)=1-10$ only, and extrapolate the fitting formula to higher FDM masses. Fig. 3.14 shows the fitting SHMF for $m_{22}=50$ compared to results derived from merger trees. It can be seen that the fitting function works well for higher FDM masses. 


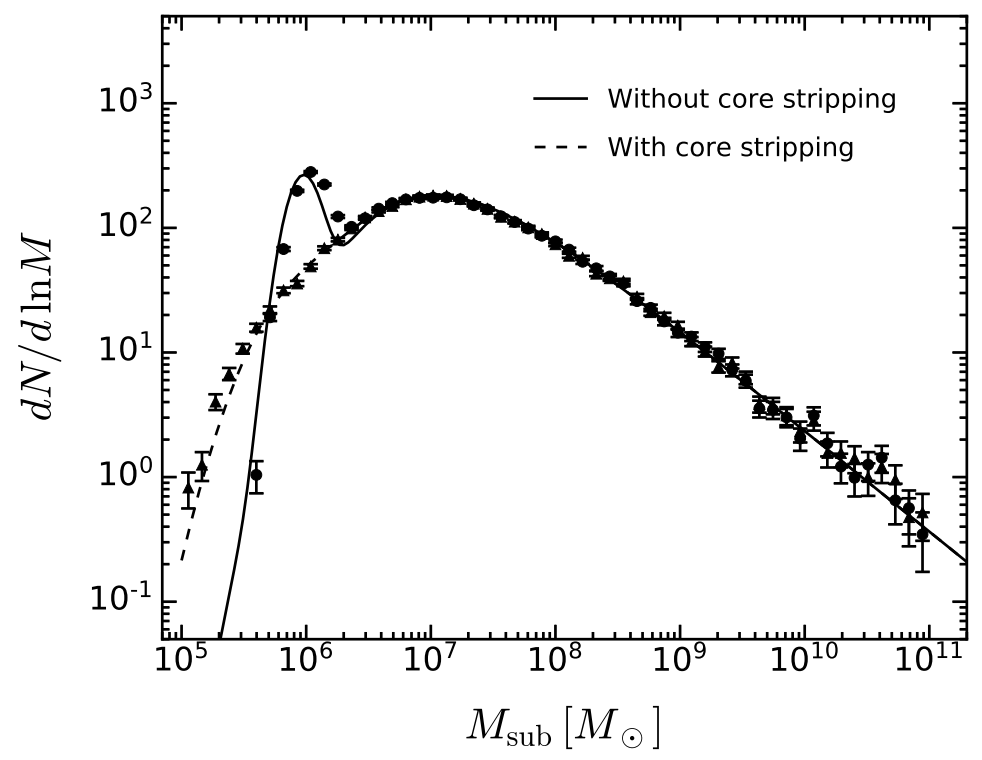

Figure 3.14: Robustness test of the fitting formula Eq. 3.22) for FDM with (solid line) and without (dashed line) tidal stripping of subhalo cores. The FDM mass $m_{22}=m_{a} /\left(10^{-22} \mathrm{eV}\right)=50$. The solid and dashed lines show the prediction from the fitting formula. Note that the fitting formula is derived from the results of merger trees with $m_{22}=1-10$ and extrapolated to $m_{22}=50$.

\subsection{Conclusions and Discussions}

In this chapter, we studied the tidal disruption of fuzzy dark matter (FDM) subhalo cores numerically by using a fourth-order pseudospectral method. First, we considered an idealized case with a spherical tidal potential. We calculated the mass loss of the core resulting from mass transfer through the tidal radius and decreasing tidal radius, and found that the contribution from the former is close to the semi-analytic prediction of [6] if the decreasing density ratio is taken into account. For lower density ratios, the mass loss due to a decreasing tidal radius dominates. In general, the core loses mass more quickly than estimated in [6] since while the core loses mass, its central density decreases, making the core more vulnerable to tidal forces (see Fig. 3.2).

We also performed three-dimensional simulations of a more realistic case where the core is evolved in the central potential of a host, treated as a small uniform sphere. In this case, it is difficult to find a well-defined tidal radius contrary to the previous case with spherical symmetry. The results show that when the solitonic core loses mass, it rapidly equilibrates to a new solitonic state with lower central density (Fig. 3.3). Even after losing a substantial fraction of its initial mass, the average core density 
profile can still be fitted by a solitonic profile. Therefore, instead of computing the mass loss rate of the matter within the tidal radius, we calculated the evolution of the core mass $M_{c} \propto \rho_{c}^{1 / 4}$ with $\rho_{c}$ the central density of the core. The mass loss rate as a function of the density ratio $\mu$ can be well described by the semi-analytic prediction of [6] if we account for a dynamically varying density ratio and an extra factor of $1 / 4$ coming from the scaling relations of solitonic cores (see Fig. 3.9).

Additionally, 6] assume a spherical tidal potential $\Phi_{t}=-\gamma \omega^{2} r^{2}$ with $\gamma=3 / 2$. They thus model the satellite as a rigid body that orbits the host with synchronous rotation which cannot be satisfied by FDM cores whose velocity field obeys $\nabla \times \mathbf{v}=0$. For a satellite without self-rotation, we have $\gamma=1$. Thus in general, $\gamma$ varies between 1 and $3 / 2$ depending on the internal velocity of the core.

Finally, we found that initially non-rotating cores acquire angular momentum in a tidal field due to tidal torque. The cores become tidally locked in less than $1 / 4 T_{\text {orbit }}$. The internal velocity field is described by an irrotational Riemann-S ellipsoid instead of a uniformly rotating rigid body satellite. With decreasing central density, the eccentricity of the ellipsoid increases and can be well approximated by a Roche ellipsoid as long as the core is approximately tidally locked. At later times, the core cannot gain sufficient additional angular momentum and begins to deviate from tidal locking. It is then rapidly disrupted.

In the three-dimensional simulations, we assumed the host to be a small uniform sphere. This is a good approximation as long as the subhalo is not too close to the center of the host. We also tested an NFW potential for the host. In this case, the tidal force is slightly smaller due to the non-vanishing density of the host at the position of the satellite. This difference can be accounted for by a redefinition of the density ratio $\mu_{\text {eff }} \equiv \rho_{c} / \rho_{\text {eff }}=\rho_{c} /\left[\bar{\rho}_{\text {host }}-\rho_{\text {host }}\left(r_{\text {sat }}\right)\right]$ and an effective orbital angular velocity $\omega_{\text {eff }}=\left(4 / 3 \pi G \rho_{\text {eff }}\right)^{1 / 2}$. With these redefinitions, the results are consistent with the approximation of the host as a uniform sphere.

With our results for the core mass loss rate, we estimated the lower bound on the mass of satellite galaxies that can be observed in the host galaxy in FDM scenarios. We calculated the minimum mass of cores that can survive for a given number of orbits in a Milky Way-like host. Its value depends on the FDM particle mass and the distance to the center of the host. We compare it with observed satellite galaxies in the Milky Way (see Fig. 3.12).

Implementing the core mass loss rate due to tidal stripping into the semi-analytic code GALACTICUS, we investigated its effects on the subhlao mass function (SHMF). We found that if we account for the core mass loss instead of simply assuming the 
core is stable against the tidal force, the first peak in SHMF found in Section 2.4.3 is smeared out. Thus it is important to include the tidal stripping of subhalo cores to get reliable SHMF for FDM. We also gave a fitting formula for FDM SHMF and showed it is robust under variation of the FDM mass. 


\section{Chapter 4}

\section{Outlook}

In this dissertation, I discussed the structure formation in the scenario of ultralight axion dark matter [which is also called "fuzzy dark matter" (FDM)] using a modified semi-analytic code for galaxy formation, GALACTICUS. I also did a few idealized simulations to study the tidal disruption of subhalo cores.

One possible extension of this work in the future would be to activate the baryonic physics in the semi-analytic code GALACTICUS and see how FDM will deviate from CDM, e.g. how the compact core in FDM halo will affect the star-formation history. It has been found in hydrodynamic simulations that adding baryonic feedback to CDM model, e.g. from AGN and supernova, could produce an inner flatter dark matter profile. Certain processes in baryonic physics may also alleviate the discrepancy in the number of satellite galaxies between CDM simulations and observations. Therefore, it is crucial to include also the baryonic physics for FDM. While it is computational infeasible at present to perform a large enough hydrodynamic simulation with FDM including baryonic feedback, semi-analytic model will provide us a useful tool to explore such possibilities and enable us to get more robust constraints on the mass of FDM (for doing this, a recalibration of the GALACTICUS is also needed).

Another interesting work is to simulate in more details different processes happening within the FDM host halo, such as dynamical friction, interference patterns and tidal streams, and to compare the results with observations from galaxy surveys, e.g. 2MASS, SDSS, and Gaia. For example, with carefully created initial conditions and the pseudo-spectral code I have, simulations can be done to study the evolution of substructure in Milky Way-like halos. Furthermore, to make the code more efficient, it will also be very interesting to combine the current pseudo-spectral code with some extension of adaptive mesh refinement algorithm. Although a full cosmological simulation would be difficult, the results from such kinds of numerical simulations can be implemented into the semi-analytic code to explore the cosmological consequences. 


\section{Appendix A}

\section{Numerical method for solving the integral equation}

In [91], the integral equation Eq. 2.86) is solved by discretizing the integral using the trapezoid rule as

$$
\int_{0}^{S_{j}} f\left(S^{\prime}\right) K\left(S_{j}, S^{\prime}\right) d S^{\prime}=\sum_{i=0}^{j-1} \frac{f\left(S_{i}\right) K\left(S_{j}, S_{i}\right)+f\left(S_{i+1}\right) K\left(S_{j}, S_{i+1}\right)}{2} \Delta S_{i} .
$$

Here, $K\left(S_{j}, S^{\prime}\right)$ is the kernel of the integral equation. To increase precision for our specific problem, we instead implement the mid-point rule:

$$
\int_{0}^{S_{j}} f\left(S^{\prime}\right) K\left(S_{j}, S^{\prime}\right) d S^{\prime}=\sum_{i=0}^{j-1} f\left(S_{i+1 / 2}\right) K\left(S_{j}, S_{i+1 / 2}\right) \Delta S_{i}
$$

The first crossing distribution $f(S)$ at $S_{j-1 / 2}$ is given by

$$
f\left(S_{j-1 / 2}\right)=\frac{1}{K\left(S_{j}, S_{j-1 / 2}\right) \Delta S_{j-1 / 2}}\left(\operatorname{erfc}\left[\frac{B\left(S_{j}\right)}{\sqrt{2 S_{j}}}\right]-\sum_{i=0}^{j-2} f\left(S_{i+1 / 2}\right) K\left(S_{j}, S_{i+1 / 2}\right) \Delta S_{i}\right) .
$$

Fig. A.1 shows that the results from the trapezoid rule display artefacts near the cutoff at lower masses. Additionally, we see unphysical oscillations at intermediate masses, which is a common behaviour when one solves integral equations using the trapezoidal rule [157, 158]. Our method avoids these artefacts. 


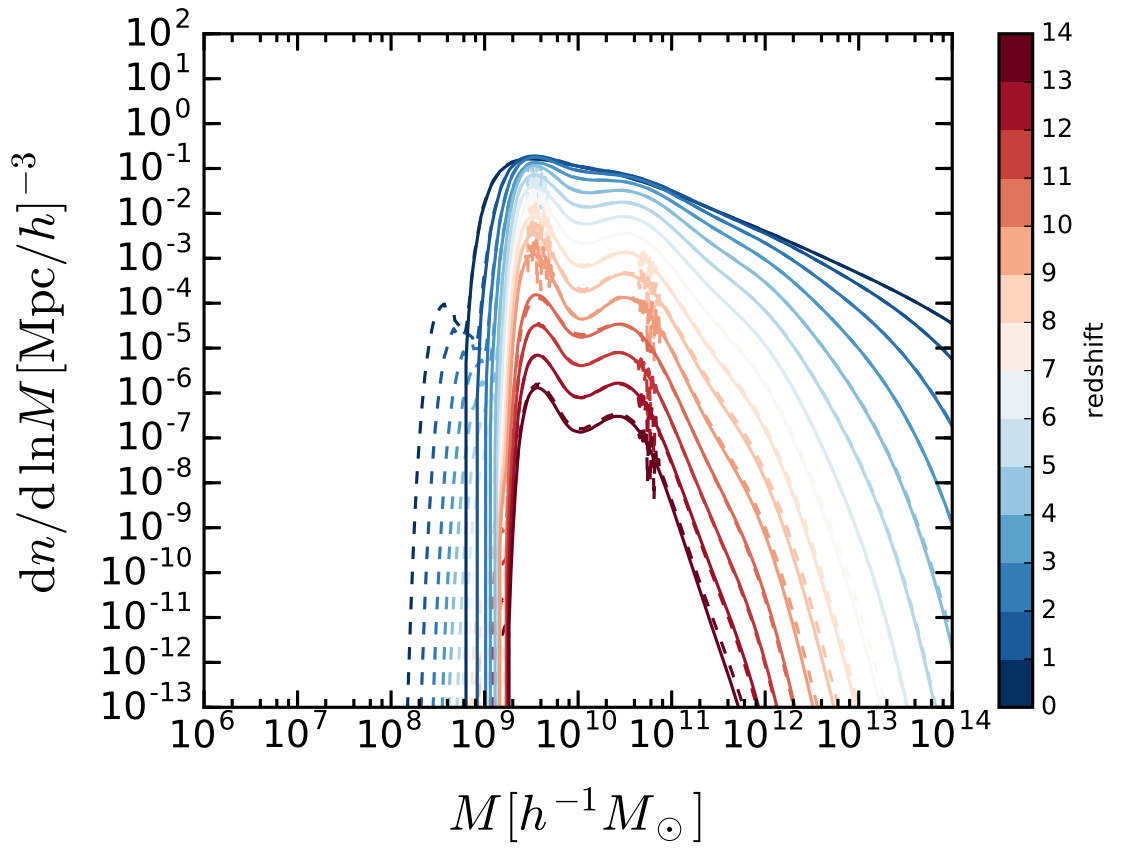

Figure A.1: HMF for FDM fraction $f=1.0$ derived using the mid-point rule (solid lines) compared to the result using the trapezoid rule (dashed lines). Redshifts range from $z=14$ to 0 . The FDM mass is set to $m_{\mathrm{a}}=10^{-22} \mathrm{eV}$. Reproduced from [1], Fig. A1. 


\section{Bibliography}

[1] Xiaolong Du, Christoph Behrens, and Jens C. Niemeyer. Substructure of fuzzy dark matter haloes. Mon. Not. Roy. Astron. Soc., 465(1):941-951, 2017.

[2] David J. E. Marsh. WarmAndFuzzy: the halo model beyond CDM. 2016.

[3] Nicola Menci, Alexander Merle, Maximilian Totzauer, Aurel Schneider, Andrea Grazian, Marco Castellano, and Norma G. Sanchez. Fundamental physics with the Hubble Frontier Fields: constraining Dark Matter models with the abundance of extremely faint and distant galaxies. Astrophys. J., 836(1):61, 2017.

[4] Hsi-Yu Schive, Ming-Hsuan Liao, Tak-Pong Woo, Shing-Kwong Wong, Tzihong Chiueh, Tom Broadhurst, and W. Y. Pauchy Hwang. Understanding the CoreHalo Relation of Quantum Wave Dark Matter from 3D Simulations. Phys. Rev. Lett., 113(26):261302, 2014.

[5] Xiaolong Du, Christoph Behrens, Jens C. Niemeyer, and Bodo Schwabe. Corehalo mass relation of ultralight axion dark matter from merger history. Phys. Rev., D95(4):043519, 2017.

[6] Lam Hui, Jeremiah P. Ostriker, Scott Tremaine, and Edward Witten. Ultralight scalars as cosmological dark matter. Phys. Rev., D95(4):043541, 2017.

[7] Xiaolong Du, Bodo Schwabe, Jens C. Niemeyer, and David Bürger. Tidal disruption of fuzzy dark matter subhalo cores. Phys. Rev., D97(6):063507, 2018 .

[8] Tanja Rindler-Daller and Paul R. Shapiro. Angular Momentum and Vortex Formation in Bose-Einstein-Condensed Cold Dark Matter Haloes. Mon. Not. Roy. Astron. Soc., 422:135-161, 2012.

[9] J. Wolf, G. D. Martinez, J. S. Bullock, M. Kaplinghat, M. Geha, R. R. Muñoz, J. D. Simon, and F. F. Avedo. Accurate masses for dispersion-supported galaxies. Mon. Not. R. Astron. Soc., 406:1220-1237, August 2010. 
[10] F. Zwicky. Die Rotverschiebung von extragalaktischen Nebeln. Helv. Phys. Acta, 6:110-127, 1933. [Gen. Rel. Grav.41,207(2009)].

[11] Vera C. Rubin, W. Kent Ford, Jr., and Norbert Thonnard. Extended rotation curves of high-luminosity spiral galaxies. IV. Systematic dynamical properties, Sa through Sc. Astrophys. J., 225:L107-L111, 1978.

[12] Gianfranco Bertone and Dan Hooper. A History of Dark Matter. Submitted to: Rev. Mod. Phys., 2016.

[13] Edwin Hubble. A relation between distance and radial velocity among extragalactic nebulae. Proc. Nat. Acad. Sci., 15:168-173, 1929.

[14] Adam G. Riess et al. Observational evidence from supernovae for an accelerating universe and a cosmological constant. Astron. J., 116:1009-1038, 1998.

[15] S. Perlmutter et al. Measurements of Omega and Lambda from 42 high redshift supernovae. Astrophys. J., 517:565-586, 1999.

[16] Graeme E. Addison, Gary Hinshaw, and Mark Halpern. Cosmological constraints from baryon acoustic oscillations and clustering of large-scale structure. Mon. Not. Roy. Astron. Soc., 436:1674-1683, 2013.

[17] Arno A. Penzias and Robert Woodrow Wilson. A Measurement of excess antenna temperature at 4080-Mc/s. Astrophys. J., 142:419-421, 1965.

[18] G. Hinshaw et al. Nine-Year Wilkinson Microwave Anisotropy Probe (WMAP) Observations: Cosmological Parameter Results. Astrophys. J. Suppl., 208:19, 2013.

[19] N. Aghanim et al. Planck 2018 results. VI. Cosmological parameters. 2018.

[20] Y. Akrami et al. Planck 2018 results. I. Overview and the cosmological legacy of Planck. 2018.

[21] Matthew R. Buckley and Annika H. G. Peter. Gravitational probes of dark matter physics. 2017.

[22] Paul Bode, Jeremiah P. Ostriker, and Neil Turok. Halo formation in warm dark matter models. Astrophys. J., 556:93-107, 2001. 
[23] Kevork Abazajian. Linear cosmological structure limits on warm dark matter. Phys. Rev. D, 73:063513, 2006.

[24] David N. Spergel and Paul J. Steinhardt. Observational evidence for selfinteracting cold dark matter. Phys. Rev. Lett., 84:3760-3763, 2000.

[25] Wayne Hu, Rennan Barkana, and Andrei Gruzinov. Cold and fuzzy dark matter. Phys. Rev. Lett., 85:1158-1161, 2000.

[26] Varun Sahni and Li-Min Wang. A New cosmological model of quintessence and dark matter. Phys. Rev. D, 62:103517, 2000.

[27] Tak-Pong Woo and Tzihong Chiueh. High-Resolution Simulation on Structure Formation with Extremely Light Bosonic Dark Matter. Astrophys. J., 697:850$861,2009$.

[28] V. Lora, Juan Magana, Argelia Bernal, F. J. Sanchez-Salcedo, and E. K. Grebel. On the mass of ultra-light bosonic dark matter from galactic dynamics. JCAP, 1202:011, 2012.

[29] Hsi-Yu Schive, Tzihong Chiueh, and Tom Broadhurst. Cosmic Structure as the Quantum Interference of a Coherent Dark Wave. Nature Phys., 10:496-499, 2014 .

[30] V. Lora and Juan Magaña. Is Sextans dwarf galaxy in a scalar field dark matter halo? JCAP, 1409:011, 2014.

[31] David J. E. Marsh and Ana-Roxana Pop. Axion dark matter, solitons and the cusp-core problem. Mon. Not. Roy. Astron. Soc., 451(3):2479-2492, 2015.

[32] Brandon Bozek, David J. E. Marsh, Joseph Silk, and Rosemary F. G. Wyse. Galaxy UV-luminosity function and reionization constraints on axion dark matter. Mon. Not. Roy. Astron. Soc., 450(1):209-222, 2015.

[33] Hsi-Yu Schive, Tzihong Chiueh, Tom Broadhurst, and Kuan-Wei Huang. Contrasting Galaxy Formation from Quantum Wave Dark Matter, $\psi$ DM, with

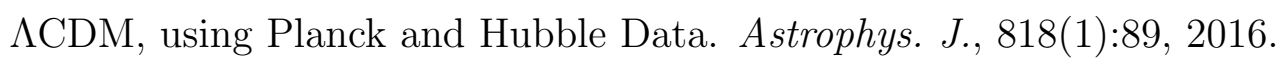

[34] Abir Sarkar, Rajesh Mondal, Subinoy Das, Shiv.K. Sethi, Somnath Bharadwaj, and David J. E. Marsh. The effects of the small-scale DM power on the cosmological neutral hydrogen $(\mathrm{HI})$ distribution at high redshifts. JCAP, 1604(04):012, 2016. 
[35] Erminia Calabrese and David N. Spergel. Ultra-light dark matter in ultra-faint dwarf galaxies. Mon. Not. Roy. Astron. Soc., 460(4):4397-4402, 2016.

[36] W.J.G. de Blok. The Core-Cusp Problem. Adv. Astron., 2010:789293, 2010.

[37] Anatoly A. Klypin, Andrey V. Kravtsov, Octavio Valenzuela, and Francisco Prada. Where are the missing Galactic satellites? Astrophys. J., 522:82-92, 1999.

[38] B. Moore, S. Ghigna, F. Governato, G. Lake, Thomas R. Quinn, J. Stadel, and P. Tozzi. Dark matter substructure within galactic halos. Astrophys. J., 524:L19-L22, 1999.

[39] C. A. Baker et al. An Improved experimental limit on the electric dipole moment of the neutron. Phys. Rev. Lett., 97:131801, 2006.

[40] David J. E. Marsh. Axion Cosmology. Phys. Rept., 643:1-79, 2016.

[41] R. D. Peccei and Helen R. Quinn. CP Conservation in the Presence of Instantons. Phys. Rev. Lett., 38:1440-1443, 1977. [,328(1977)].

[42] Cumrun Vafa and Edward Witten. Parity Conservation in QCD. Phys. Rev. Lett., 53:535, 1984.

[43] Steven Weinberg. A new light boson? Phys. Rev. Lett., 40:223-226, Jan 1978.

[44] F. Wilczek. Problem of strong $p$ and $t$ invariance in the presence of instantons. Phys. Rev. Lett., 40:279-282, Jan 1978.

[45] John Preskill, Mark B. Wise, and Frank Wilczek. Cosmology of the Invisible Axion. Phys. Lett., 120B:127-132, 1983.

[46] L. F. Abbott and P. Sikivie. A Cosmological Bound on the Invisible Axion. Phys. Lett., 120B:133-136, 1983.

[47] Michael Dine and Willy Fischler. The Not So Harmless Axion. Phys. Lett., 120B:137-141, 1983.

[48] Pierre Sikivie. Axion Cosmology. Lect. Notes Phys., 741:19-50, 2008. $[, 19(2006)]$.

[49] Michael S. Turner. Coherent Scalar Field Oscillations in an Expanding Universe. Phys. Rev., D28:1243, 1983. 
[50] William H. Press, Barbara S. Ryden, and David N. Spergel. Single Mechanism for Generating Large Scale Structure and Providing Dark Missing Matter. Phys. Rev. Lett., 64:1084, 1990.

[51] Sang-Jin Sin. Late time cosmological phase transition and galactic halo as Bose liquid. Phys. Rev., D50:3650-3654, 1994.

[52] Jeremy Goodman. Repulsive dark matter. New Astron., 5:103, 2000.

[53] P. J. E. Peebles. Fluid dark matter. Astrophys. J., 534:L127, 2000.

[54] Luca Amendola and Riccardo Barbieri. Dark matter from an ultra-light pseudoGoldsone-boson. Phys. Lett. B, 642:192-196, 2006.

[55] David J. E. Marsh and Joe Silk. A Model For Halo Formation With Axion Mixed Dark Matter. Mon. Not. Roy. Astron. Soc., 437(3):2652-2663, 2014.

[56] Erminia Calabrese and David N. Spergel. Ultra-light dark matter in ultra-faint dwarf galaxies. Mon. Not. Roy. Astron. Soc., 460(4):4397-4402, 2016.

[57] Alma X. GonzÃ !'lez-Morales, David J. E. Marsh, Jorge Pe $\tilde{A} \pm$ arrubia, and Luis A. Ure $\tilde{A} \pm a-L \tilde{A} p e z$. Unbiased constraints on ultralight axion mass from dwarf spheroidal galaxies. Mon. Not. Roy. Astron. Soc., 472(2):1346-1360, 2017.

[58] Michael Boylan-Kolchin, James S. Bullock, and Manoj Kaplinghat. Too big to fail? the puzzling darkness of massive milky way subhaloes. Mon. Not. Roy. Astron. Soc., 415(1):L40-L44, 2011.

[59] Edward Witten. Some Properties of O(32) Superstrings. Phys. Lett. B, 149:351$356,1984$.

[60] Peter Svrcek and Edward Witten. Axions In String Theory. J. High Energy Phys., 06:051, 2006.

[61] Asimina Arvanitaki, Savas Dimopoulos, Sergei Dubovsky, Nemanja Kaloper, and John March-Russell. String Axiverse. Phys. Rev. D, 81:123530, 2010.

[62] Bodo Schwabe, Jens C. Niemeyer, and Jan F. Engels. Simulations of solitonic core mergers in ultralight axion dark matter cosmologies. Phys. Rev., D94(4):043513, 2016. 
[63] Jan Veltmaat and Jens C. Niemeyer. Cosmological particle-in-cell simulations with ultralight axion dark matter. Phys. Rev., D94(12):123523, 2016.

[64] Shu-Rong Chen, Hsi-Yu Schive, and Tzihong Chiueh. Jeans Analysis for Dwarf Spheroidal Galaxies in Wave Dark Matter. Mon. Not. Roy. Astron. Soc., 468(2):1338-1348, 2017.

[65] P. S. Corasaniti, S. Agarwal, D. J. E. Marsh, and S. Das. Constraints on dark matter scenarios from measurements of the galaxy luminosity function at high redshifts. Phys. Rev., D95(8):083512, 2017.

[66] Rene Hlozek, Daniel Grin, David J. E. Marsh, and Pedro G. Ferreira. A search for ultralight axions using precision cosmological data. Phys. Rev., D91(10):103512, 2015.

[67] Matteo Viel, George D. Becker, James S. Bolton, and Martin G. Haehnelt. Warm dark matter as a solution to the small scale crisis: New constraints from high redshift Lyman- forest data. Phys. Rev. D, 88:043502, 2013.

[68] Aurel Schneider. Astrophysical constraints on resonantly produced sterile neutrino dark matter. JCAP, 1604(04):059, 2016.

[69] Vid Iršič, Matteo Viel, Martin G. Haehnelt, James S. Bolton, and George D. Becker. First constraints on fuzzy dark matter from Lyman- $\alpha$ forest data and hydrodynamical simulations. Phys. Rev. Lett., 119(3):031302, 2017.

[70] Takeshi Kobayashi, Riccardo Murgia, Andrea De Simone, Vid Iršič, and Matteo Viel. Lyman- $\alpha$ constraints on ultralight scalar dark matter: Implications for the early and late universe. Phys. Rev. D, 96(12):123514, 2017.

[71] Jiajun Zhang, Jui-Lin Kuo, Hantao Liu, Yue-Lin Sming Tsai, Kingman Cheung, and Ming-Chung Chu. The Importance of Quantum Pressure of Fuzzy Dark Matter on Lyman-Alpha Forest. Astrophys. J., 863:73, 2018.

[72] Andrea V. Maccio, Sinziana Paduroiu, Donnino Anderhalden, Aurel Schneider, and Ben Moore. Cores in warm dark matter haloes: a Catch 22 problem. Mon. Not. R. Astron. Soc., 424:1105-1112, 2012.

[73] Andrew R. Wetzel, Philip F. Hopkins, Ji-hoon Kim, Claude-Andre FaucherGiguere, Dusan Keres, and Eliot Quataert. Reconciling dwarf galaxies with 
LCDM cosmology: Simulating a realistic population of satellites around a Milky Way-mass galaxy. Astrophys. J., 827(2):L23, 2016.

[74] M. I. Scrimgeour, T. Davis, C. Blake, J. B. James, G. B. Poole, L. StaveleySmith, S. Brough, M. Colless, C. Contreras, W. Couch, S. Croom, D. Croton, M. J. Drinkwater, K. Forster, D. Gilbank, M. Gladders, K. Glazebrook, B. Jelliffe, R. J. Jurek, I.-h. Li, B. Madore, D. C. Martin, K. Pimbblet, M. Pracy, R. Sharp, E. Wisnioski, D. Woods, T. K. Wyder, and H. K. C. Yee. The WiggleZ Dark Energy Survey: the transition to large-scale cosmic homogeneity. Mon. Not. Roy. Astron. Soc., 425:116-134, September 2012.

[75] Pierros Ntelis et al. Exploring cosmic homogeneity with the BOSS DR12 galaxy sample. JCAP, 1706(06):019, 2017.

[76] D. J. Fixsen, E. S. Cheng, J. M. Gales, John C. Mather, R. A. Shafer, and E. L. Wright. The Cosmic Microwave Background spectrum from the full COBE FIRAS data set. Astrophys. J., 473:576, 1996.

[77] P. A. R. Ade et al. Planck 2015 results. XIII. Cosmological parameters. Astron. Astrophys., 594:A13, 2016.

[78] David J. E. Marsh and Pedro G. Ferreira. Ultra-Light Scalar Fields and the Growth of Structure in the Universe. Phys. Rev., D82:103528, 2010.

[79] Bharat Ratra. Expressions for linearized perturbations in a massive scalar field dominated cosmological model. Phys. Rev., D44:352-364, 1991.

[80] S. Weinberg. Cosmology. Cosmology. OUP Oxford, 2008.

[81] L. Arturo Urea-Lpez and Alma X. Gonzalez-Morales. Towards accurate cosmological predictions for rapidly oscillating scalar fields as dark matter. JCAP, 1607(07):048, 2016.

[82] Jai-chan Hwang and Hyerim Noh. Axion as a Cold Dark Matter candidate. Phys. Lett., B680:1-3, 2009.

[83] Chan-Gyung Park, Jai-chan Hwang, and Hyerim Noh. Axion as a cold dark matter candidate: low-mass case. Phys. Rev., D86:083535, 2012.

[84] Hyerim Noh, Chan-Gyung Park, and Jai-chan Hwang. Axion as a Cold Dark Matter Candidate: Proof to Second order. Phys. Lett., B726:559-563, 2013. 
[85] M. Khlopov, B. A. Malomed, and Ia. B. Zeldovich. Gravitational instability of scalar fields and formation of primordial black holes. Mon. Not. Roy. Astron. Soc., 215:575-589, 1985.

[86] Erwin Madelung. Eine anschauliche deutung der gleichung von schrödinger. Naturwissenschaften, 14:1004, 1926.

[87] J. Binney and S. Tremaine. Galactic Dynamics. Princeton series in astrophysics. Princeton University Press, 1987.

[88] H. Mo, F. van den Bosch, and S. White. Galaxy Formation and Evolution. Cambridge University Press, 2010.

[89] William H. Press and Paul Schechter. Formation of galaxies and clusters of galaxies by selfsimilar gravitational condensation. Astrophys. J., 187:425-438, 1974.

[90] Rennan Barkana, Zoltan Haiman, and Jeremiah P. Ostriker. Constraints on warm dark matter from cosmological reionization. Astrophys. J., 558:482, 2001.

[91] Andrew J. Benson, Arya Farahi, Shaun Cole, Leonidas A. Moustakas, Adrian Jenkins, Mark Lovell, Rachel Kennedy, John Helly, and Carlos Frenk. Dark Matter Halo Merger Histories Beyond Cold Dark Matter: I - Methods and Application to Warm Dark Matter. Mon. Not. Roy. Astron. Soc., 428:1774, 2013.

[92] Jan Veltmaat, Jens C. Niemeyer, and Bodo Schwabe. Formation and structure of ultralight bosonic dark matter halos. Phys. Rev., D98(4):043509, 2018.

[93] P. Monaco. The Cosmological Mass Function. Fund. Cosm. Phys., 19:157-317, 1998.

[94] R. I. Epstein. Proto-galactic perturbations. Mon. Not. Roy. Astron. Soc., 205:207-229, October 1983.

[95] R. I. Epstein. Galaxies from Poisson fluctuations. Astrophys. J., 281:545-553, June 1984.

[96] James M. Bardeen, J. R. Bond, Nick Kaiser, and A. S. Szalay. The Statistics of Peaks of Gaussian Random Fields. Astrophys. J., 304:15-61, 1986. 
[97] R. Schaeffer and J. Silk. Cold, warm, or hot dark matter - Biased galaxy formation and pancakes. Astrophys. J., 332:1-16, September 1988.

[98] J. A. Peacock and A. F. Heavens. Alternatives to the press-schechter cosmological mass function. Monthly Notices of the Royal Astronomical Society, 243(1):133-143, 1990.

[99] J. R. Bond, S. Cole, G. Efstathiou, and Nick Kaiser. Excursion set mass functions for hierarchical Gaussian fluctuations. Astrophys. J., 379:440, 1991.

[100] Cedric G. Lacey and Shaun Cole. Merger rates in hierarchical models of galaxy formation. Mon. Not. Roy. Astron. Soc., 262:627-649, 1993.

[101] Aurel Schneider, Robert E. Smith, and Darren Reed. Halo Mass Function and the Free Streaming Scale. Mon. Not. Roy. Astron. Soc., 433:1573, 2013.

[102] Aurel Schneider. Structure formation with suppressed small-scale perturbations. Mon. Not. Roy. Astron. Soc., 451(3):3117-3130, 2015.

[103] Cedric Lacey and Shanu Cole. Merger rates in hierarchical models of galaxy formation - ii. comparison with n-body simulations. Monthly Notices of the Royal Astronomical Society, 271(3):676-692, Dec 1994.

[104] Rachel S. Somerville, Gerard Lemson, Tsafrir S. Kolatt, and Avishai Dekel. Evaluating semi-analytic halo merging histories. Mon. Not. Roy. Astron. Soc., 316:479, 2000.

[105] Shaun Cole, Cedric G. Lacey, Carlton M. Baugh, and Carlos S. Frenk. Hierarchical galaxy formation. Mon. Not. Roy. Astron. Soc., 319:168, 2000.

[106] Andrew J Benson, Chris Cannella, and Shaun Cole. Achieving convergence in galaxy formation models by augmenting n-body merger trees. Computational Astrophysics and Cosmology, 3(1), Aug 2016.

[107] Rachel S. Somerville and Tsafrir S. Kolatt. How to plant a merger tree. Mon. Not. Roy. Astron. Soc., 305:1-14, 1999.

[108] G. Kauffmann and S. D. M. White. The merging history of dark matter haloes in a hierarchical universe. Mon. Not. Roy. Astron. Soc., 261:921-928, 1993.

[109] Jun Zhang, Onsi Fakhouri, and Chung-Pei Ma. How to Grow a Healthy Merger Tree. Mon. Not. Roy. Astron. Soc., 389:1521-1538, 2008. 
[110] Eyal Neistein and Avishai Dekel. Constructing Merger Trees that Mimic NBody Simulations. Mon. Not. Roy. Astron. Soc., 383:615, 2008.

[111] Fangzhou Jiang and Frank C. van den Bosch. Generating Merger Trees for Dark Matter Haloes: A Comparison of Methods. Mon. Not. Roy. Astron. Soc., 440(1):193-207, 2014.

[112] S. D. M. White and M. J. Rees. Core condensation in heavy halos: a twostage theory for galaxy formation and clustering. Monthly Notices of the Royal Astronomical Society, 183(3):341-358, Jul 1978.

[113] S. Cole. Modeling galaxy formation in evolving dark matter halos. Astrophys. J., 367:45-53, January 1991.

[114] Simon D. M. White and Carlos S. Frenk. Galaxy formation through hierarchical clustering. Astrophys. J., 379:52-79, 1991.

[115] C. Lacey and J. Silk. Tidally triggered galaxy formation. I - Evolution of the galaxy luminosity function. Astrophys. J., 381:14-32, November 1991.

[116] Carlton M. Baugh. A primer on hierarchical galaxy formation: The semianalytical approach. Rept. Prog. Phys., 69:3101-3156, 2006.

[117] Andrew J. Benson. Galaxy Formation Theory. Phys. Rept., 495:33-86, 2010.

[118] Rachel S. Somerville and Romeel Dav. Physical Models of Galaxy Formation in a Cosmological Framework. Ann. Rev. Astron. Astrophys., 53:51-113, 2015.

[119] Darren J. Croton, Volker Springel, Simon D. M. White, G. De Lucia, C. S. Frenk, L. Gao, A. Jenkins, G. Kauffmann, J. F. Navarro, and N. Yoshida. The many lives of active galactic nuclei: cooling flows, black holes and the luminosities and colours of galaxies. Monthly Notices of the Royal Astronomical Society, 365(1):11-28, Jan 2006.

[120] D. J. Croton, V. Springel, S. D. M. White, G. De Lucia, C. S. Frenk, L. Gao, A. Jenkins, G. Kauffmann, J. F. Navarro, and N. Yoshida. Erratum: The many lives of active galactic nuclei: cooling flows, black holes and the luminosities and colours of galaxies. Monthly Notices of the Royal Astronomical Society, 367(2):864-864, Apr 2006. 
[121] Rachel S. Somerville, Philip F. Hopkins, Thomas J. Cox, Brant E. Robertson, and Lars Hernquist. A semi-analytic model for the co-evolution of galaxies, black holes and active galactic nuclei. Monthly Notices of the Royal Astronomical Society, 391(2):481-506, Dec 2008.

[122] Andrew J. Benson. Galacticus: A Semi-Analytic Model of Galaxy Formation. New Astron., 17:175-197, 2012.

[123] Anthony R. Pullen, Andrew J. Benson, and Leonidas A. Moustakas. Nonlinear evolution of dark matter subhalos and applications to warm dark matter. Astrophys. J., 792:24, 2014.

[124] Philip Mocz, Mark Vogelsberger, Victor H. Robles, JesÃs Zavala, Michael Boylan-Kolchin, Anastasia Fialkov, and Lars Hernquist. Galaxy formation with BECDM: I. Turbulence and relaxation of idealized haloes. Mon. Not. Roy. Astron. Soc., 471(4):4559-4570, 2017.

[125] Philip Mocz and Sauro Succi. Numerical solution of the nonlinear Schrdinger equation using smoothed-particle hydrodynamics. Phys. Rev., E91(5):053304, 2015.

[126] David J. E. Marsh. Nonlinear hydrodynamics of axion dark matter: Relative velocity effects and quantum forces. Phys. Rev., D91(12):123520, 2015.

[127] M. Nori and M. Baldi. AX-GADGET: a new code for cosmological simulations of Fuzzy Dark Matter and Axion models. Mon. Not. Roy. Astron. Soc., 478:3935, 2018.

[128] Antony Lewis, Anthony Challinor, and Anthony Lasenby. Efficient computation of CMB anisotropies in closed FRW models. Astrophys. J., 538:473-476, 2000.

[129] Ravi K. Sheth and Giuseppe Tormen. Large scale bias and the peak background split. Mon. Not. Roy. Astron. Soc., 308:119, 1999.

[130] Ravi K. Sheth, H. J. Mo, and Giuseppe Tormen. Ellipsoidal collapse and an improved model for the number and spatial distribution of dark matter haloes. Mon. Not. Roy. Astron. Soc., 323:1, 2001.

[131] S. F. Schandarin and Ya. B. Zeldovich. The Large scale structure of the universe: Turbulence, intermittency, structures in a selfgravitating medium. Rev. Mod. Phys., 61:185-220, 1989. 
[132] Ravi K. Sheth and Giuseppe Tormen. An Excursion Set Model of Hierarchical Clustering : Ellipsoidal Collapse and the Moving Barrier. Mon. Not. Roy. Astron. Soc., 329:61, 2002.

[133] Hannah Parkinson, Shaun Cole, and John Helly. Generating Dark Matter Halo Merger Trees. Mon. Not. Roy. Astron. Soc., 383:557, 2008.

[134] G. L. Bryan and M. L. Norman. Statistical properties of x-ray clusters: Analytic and numerical comparisons. Astrophys. J., 495:80, 1998.

[135] Aurel Schneider, Robert E. Smith, Andrea V. Maccio, and Ben Moore. Nonlinear Evolution of Cosmological Structures in Warm Dark Matter Models. Mon.Not.Roy.Astron.Soc., 424:684, 2012.

[136] Liang Gao, Julio F. Navarro, Shaun Cole, Carlos Frenk, Simon D. M. White, Volker Springel, Adrian Jenkins, and Angelo F. Neto. The redshift dependence of the structure of massive LCDM halos. Mon. Not. Roy. Astron. Soc., 387:536, 2008 .

[137] Julio F. Navarro, Carlos S. Frenk, and Simon D. M. White. The Structure of cold dark matter halos. Astrophys. J., 462:563-575, 1996.

[138] Julio F. Navarro, Carlos S. Frenk, and Simon D.M. White. A Universal density profile from hierarchical clustering. Astrophys.J., 490:493-508, 1997.

[139] C. Y. Jiang, Y. P. Jing, A. Faltenbacher, W. P. Lin, and Cheng Li. A fitting formula for the merger timescale of galaxies in hierarchical clustering. Astrophys. J., 675:1095, 2008.

[140] Michael Boylan-Kolchin, Chung-Pei Ma, and Eliot Quataert. Dynamical Friction and Galaxy Merging Timescales. Mon. Not. Roy. Astron. Soc., 383:93, 2008 .

[141] Andrew R. Wetzel and Martin White. What determines satellite galaxy disruption? Mon. Not. Roy. Astron. Soc., 403:1072, 2010.

[142] lvaro Villalobos, Gabriella De Lucia, Simone Weinmann, Stefano Borgani, and Giuseppe Murante. An improved prescription for merger time-scales from controlled simulations. Mon. Not. Roy. Astron. Soc., 433:49, 2013. 
[143] Andrew J. Benson. Orbital parameters of infalling dark matter substructures. Mon. Not. Roy. Astron. Soc., 358:551-562, 2005. [Erratum: Mon. Not. Roy. Astron. Soc.364,1104 (2005)].

[144] Andrew J. Benson. Erratum: Orbital parameters of infalling dark matter substructures. Mon. Not. Roy. Astron. Soc., 364(3):1104-1104, 2005.

[145] Subrahmanyan Chandrasekhar. Dynamical Friction. I. General Considerations: the Coefficient of Dynamical Friction. Astrophys. J., 97:255, 1943.

[146] I. King. The structure of star clusters. I. an empirical density law. Astrophys. J., 67:471, October 1962.

[147] Oleg Y. Gnedin, Lars Hernquist, and Jeremiah P. Ostriker. Tidal shocking by extended mass distributions. Astrophys. J., 514:109-118, 1999.

[148] James E. Taylor and Arif Babul. The Dynamics of sinking satellites around disk galaxies: A Poor man's alternative to high - resolution numerical simulations. Astrophys. J., 559:716-735, 2001.

[149] Arya Farahi and Andrew J. Benson. Excursion Set Theory for Correlated Random Walks. Mon. Not. Roy. Astron. Soc., 433(4):3428-3439, 2013.

[150] F. Siddhartha Guzman and L. Arturo Urena-Lopez. Evolution of the Schrodinger-Newton system for a selfgravitating scalar field. Phys. Rev., D69:124033, 2004.

[151] Frank C. van den Bosch, Go Ogiya, Oliver Hahn, and Andreas Burkert. Disruption of dark matter substructure: fact or fiction? Mon. Not. R. Astron. Soc., 474(3):3043-3066, 2018.

[152] Frank C. van den Bosch and Go Ogiya. Dark Matter Substructure in Numerical Simulations: A Tale of Discreteness Noise, Runaway Instabilities, and Artificial Disruption. Mon. Not. Roy. Astron. Soc., 2018.

[153] Robert I. McLachlan. On the numerical integration of ordinary differential equations by symmetric composition methods. SIAM Journal on Scientific Computing, 16(1):151-168, 1995.

[154] Édouard A. Roche. La figure d'une masse fluide soumise à l'attraction d'un point éloigné. Mém. de l'Acad. des Sci. de Montpellier, 1:243, 1850. 
[155] James S. Bullock, Avishai Dekel, Tsafrir S. Kolatt, Andrey V. Kravtsov, Anatoly A. Klypin, Cristiano Porciani, and Joel R. Primack. A Universal angular momentum profile for galactic halos. Astrophys. J., 555:240-257, 2001.

[156] Volker Springel, Jie Wang, Mark Vogelsberger, Aaron Ludlow, Adrian Jenkins, Amina Helmi, Julio F. Navarro, Carlos S. Frenk, and Simon D. M. White. The Aquarius Project: the subhalos of galactic halos. Mon. Not. Roy. Astron. Soc., 391:1685-1711, 2008.

[157] J. G. Jones. On the numerical solution of convolution integral equations and systems of such equations. Mathematics of Computation, 15(74):131-142, 1961.

[158] P. Linz. Numerical methods for volterra integral equations of the first kind. The Computer Journal, 12(4):393-397, 1969. 